\title{
OBSERVATIONS OF DWARF NOVAE
}

\author{
B. W A R N E R \\ Dept. of Astronomy, University of Cape Town, South Africa
}

\begin{abstract}
Spectroscopic and photometric observations relevant to the structure of classical novae, recurrent novae and dwarf novae are collected together. Details are given of optical, infrared, satellite ultraviolet, X-ray and radio observations. Most of these can be understood in terms of the hot spot model of cataclysmic variable stars. Estimates are made of luminosities, temperatures and rates of mass transfer. Observations made during eruptions of dwarf novae imply large changes taking place in the accretion disc.
\end{abstract}

\section{Introduction}

This review is concerned with the properties of those objects known as Cataclysmic Variables (CV's, sometimes also called eruptive binaries). This class consists of the classical novae, the recurrent novae, the dwarf novae and the nova-like variables (sometimes called UX UMa stars) which in many cases are probably classical novae which were historically unrecorded. Closely associated with these objects are the symbiotic variables, in which outbursts are less abrupt, and possibly some peculiar, hot, subluminous, low amplitude variables such as HL Tau-76. The X-ray sources of the Sco X-1 and Cyg X-2 type may also be related.

Following the early work by Walker $(1954,1956)$, which showed that DQ Her (Nova Herculis 1934) is an eclipsing binary, and by Joy (1954), who demonstrated that the nova-like variable $\mathrm{AE}$ Aqr is a spectroscopic binary, there have been numerous discoveries of duplicity among the CV's. A review of the observational aspects of this work has been given by Mumford (1967a); reviews of both observations and interpretations have been given by Kraft $(1963,1966)$ and Smak (1971c) and a review of theoretical aspects has been given by Faulkner (1974). In this review we are mostly concerned with collection and interpretation of observational data.

A current list of CV's with known binary periods, derived from periodic light or radial velocity variations, is given in Table I. To these we may add EY Cyg (Kraft, 1962) and V1017 Sgr (Kraft, 1964a), both of which have composite spectra, in which no periodic variations have been detected and are thus thought to be pole-on. Also, RT Ser and RR Tel have companions detectable in the infrared (see below). It is essentially true to say that all CV's that have been well studied have proved to be double. SU UMa (Kraft, 1963), CP Lac (Kraft, 1964b), and MV Lyr (Walker, 1966) show radial velocity variations but no periods have yet been determined. CN Ori shows periodic light variations (Warner and Brickhill, 1975) with a period probably near $6 \mathrm{~h}$.

Several general facts emerge from Table $I$. There is a strong preference for short and ultra-short periods. There appears to be a significant deficiency of periods between $1^{\text {h }}$ $47^{\mathrm{m}}$ and $3^{\mathrm{h}} 12^{\mathrm{m}}$. The only star to fall in this gap is MV Lyr, which may have a period near $2^{\text {h }}$ (Walker, 1966). T CrB is the only binary listed with a long period, but there are undoubtedly some selection effects applying at this end of the list because of the difficulty of detecting small radial velocity changes in faint stars. Any long period system will probably contain a late-type giant or supergiant. Feast and Glass (1974) find that the $\mathrm{M}$ giant in $\mathrm{TCrB}$ is readily seen in infrared photometry. They have demonstrated the 
TABLE I

Cataclysmic variables with known orbital periods

\begin{tabular}{|c|c|c|c|c|c|}
\hline & & $V$ & $P$ & $\begin{array}{l}\text { Sp } \\
\text { (secondary) }\end{array}$ & Ref. \\
\hline $\mathrm{T}$ CrB & RN & 10.5 & $227^{\mathrm{d}} \cdot 6$ & gM3 & 1 \\
\hline GK Per & $\mathbf{N}$ & 13.0 & $16^{\mathrm{h}} 26^{\mathrm{m}}$ & $\mathrm{K} 21 \mathrm{Vp}$ & $2,3,9,36$ \\
\hline BV Cen & DN & 12.9 & 1438 & dG: & 4 \\
\hline AE Aqr & NL & 11.5 & 953 & $\mathrm{~K} 2 \mathrm{~V}$ & 5,6 \\
\hline RU Peg & DN & 12.8 & 854 & G81Vn & 7 \\
\hline EM Cyg & DN & 12.9 & 700 & G or $\mathrm{K}$ & 8,9 \\
\hline Z Cam & DN & 13.6 & 656 & $\mathrm{dG} 1$ & 10 \\
\hline SS Cyg & DN & 12.1 & 638 & dG5 & 7,11 \\
\hline RW Tri & NL & 12.9 & 534 & - & 13 \\
\hline RX And & DN & 12.6 & 505 & - & 7,9 \\
\hline T Aur & $\mathbf{N}$ & 14.9 & 454 & - & $14,15,9$ \\
\hline UX UMa & NL & 12.3 & 443 & - & 16 \\
\hline DQ Her & $\mathrm{N}$ & 14.2 & 439 & - & $2,17,18$ \\
\hline SS Aur & DN & 14.7 & 420 & - & 19 \\
\hline U Gem & DN & 14.5 & 410 & - & $7,20,21$ \\
\hline WW Cet & DN & 13.6 & 350 & - & 22,9 \\
\hline RR Pic & $\mathrm{N}$ & 12.0 & 329 & - & 23,24 \\
\hline VZ Scl & NL & 15.6 & 328 & - & 25 \\
\hline V 603 Aql & $\mathbf{N}$ & 11.5 & 319 & - & 2,9 \\
\hline TT Ari & NL & 10.6 & 312 & - & 12,35 \\
\hline VW Hyi & DN & 13.4 & 147 & - & 34 \\
\hline Z Cha & DN & 15.2 & 147 & - & 23,33 \\
\hline VV Pup & NL & 14.5 & 140 & - & 27,28 \\
\hline EX Hya & DN & 13.7 & 139 & - & 29 \\
\hline V 436 Cen & DN & 15.5 & 132 & - & 26 \\
\hline WZ Sge & DN & 15.2 & 122 & - & 2,30 \\
\hline AM CVn & NL & 14.2 & 18 & - & 31,32 \\
\hline
\end{tabular}

References to Table I

1. Kraft (1958)

2. Kraft (1964a)

3. Paczynski (1965a)

4. Vogt (private communication)

5. Joy (1954)

6. G.Chincarini (private communication)

7. Kraft (1962)

8. Mumford and Krzeminski (1969)

9. Robinson (1974)

10. Kraft et al. (1969)

11. Walker and Chincarini (1968)

12. Smak and Stepien (1968)

13. Walker (1963a)

14. Walker (1963b)

15. Mumford (1967b)

16. Walker and Herbig (1954)

17. Greenstein and Kraft (1959)

18. Walker (1961)
19. Kraft and Luyten (1965)

20. Krzeminski (1965)

21. Warner and Nather (1971)

22. Kraft (1964b)

23. Mumford (1971)

24. Van Houten (1966)

25. Krzeminski (1966)

26. Warner (1975b)

27. Herbig (1960b)

28. Warner and Nather (1972a)

29. Mumford (1967c)

30. Krzeminski and Smak (1971)

31. Smak (1967)

32. Warner and Robinson (1972a)

33. Warner (1974a)

34. Warner (1975a)

35. Cowley et al. (1975)

36. Gallagher and Oinas (1974)

presence of red giant secondaries in RT Ser and RR Tel, both of which are slow novae. RS Oph may also have a red giant companion. From the number of CV's already studied, however, it seems unlikely that there are many long period objects.

Perhaps the most striking feature of Table $I$ is that, with the exception of recurrent 
novae, the nature of the outbursts is independent of the binary period. This indicates that we probably have to look elsewhere than masses or separation of the components for an explanation of the different outburst timescales. More importantly, it also suggests that it may be possible to find a unified model for all the cataclysmic variables. Such a model should aim to understand the light, spectral, colour and period variations observed at normal (minimum) light, and why outbursts occur. It should also explain the other correlation evident in Table I: why the secondary spectrum is visible only in those systems with periods longer than $6 \mathrm{~h}$. Note that this correlation also is independent of outburst properties. Before discussing such a model let us first review some of the general properties of the cataclysmic variable stars as observed at minimum light.

\section{Spectra at Minimum Light}

The pioneering studies of the spectra of nova remnants by Humason (1938) at Mount Wilson and of dwarf novae by Elvey and Babcock (1943) at McDonald Observatory, although of low resolution, resulted in the discovery of the essential peculiarities of the cataclysmic variables. With a few exceptions, all these objects showed Balmer emission superimposed on a continuous spectrum. In addition, many objects showed He I, He II and $\mathrm{Ca}$ II emission. In a few stars, Humason recorded only continuous spectra, but later studies at higher resolution show that at least one of these objects (V 1017 Sgr) has weak emission lines.

Later surveys, mostly with the improved resolution at the 200-in. telescope (Kraft, 1958, 1962; Greenstein, 1960), confirmed the earlier work, added more details of the emission lines (e.g. the presence of doubled emission lines in some systems, the presence of the $\lambda 4650 \mathrm{C}$ III-N III group of lines) and resulted in the discovery of broad absorption lines in three objects (WZ Sge, DI Lac, UX UMa) and of the absorption spectra of G or $\mathrm{K}$ stars in a number of others. We have collected together available descriptions of the spectra of CV's at minimum light and these are summarized in Table II. Sco X-1 and Cyg $\mathrm{X}-2$ have been included for comparison purposes. In most of the objects listed the presence of an overlying continuum is also established, and in several objects the Balmer discontinuity appears in emission.

From the results shown in Table II, two clear correlations stand out: The occurrence of the $\lambda 4650$ group of lines is restricted to novae, and He II emission appears strongly only in novae and recurrent novae. Weak He II emission exists in the Z Cam type dwarf novae RX And and SY Cnc. With this correlation in mind, it is probable that nova-like variables, such as VV Pup and VZ Scl, are ordinary novae that had prehistoric eruptions.

Thus we have a well-defined relationship between the ionization level of the emission regions and the nature of the outbursts. It has been suggested (McLaughlin, 1953) that the intensity of He II may fall (relative to $\mathrm{H}$ ) following the nova outburst, resulting in the absence of He II emission in very old novae. This would then link the presence of He II and $\lambda 4650$ emissions to the after-effects of the energetic nova outburst. However, as Greenstein (1960) has already pointed out, the great strength of He II emission in Nova Oph 1848 shows that not all novae obey this suggestion. The presence of He II emission in some nova-like variables also argues against this suggestion. We conclude that, despite the absence of any correlation between outburst properties and masses or separations pointed out in the previous section, there is a correlation between the outburst 
TABLE II

Line spectra of cataclysmic variables (Emission unless otherwise noted)

\begin{tabular}{|c|c|c|c|}
\hline \multicolumn{4}{|c|}{ (i) Spectra of novae at minimum light } \\
\hline V368 Aql & (1936) & $\mathrm{H}, \mathrm{He}$ II broad and moderately strong & (1) \\
\hline V603 Aq1 & (1918) & Strong $\mathrm{H}, \mathrm{He}$ II, weak He I, $\lambda 4650$ & (2) (3) \\
\hline T Aur & (1891) & Weak $\mathrm{H}, \mathrm{He}$ II & (4) \\
\hline V476 Cyg & (1920) & $\mathrm{H}, \mathrm{He}$ II broad (double), weak & (3) (4) (5) \\
\hline Q Cyg & (1876) & Weak, narrow $\mathrm{H}$ & (3) (4) \\
\hline HR Del & (1967) & Continuous at low dispersion & (47) \\
\hline DM Gem & (1903) & Continuous at low dispersion & (4) \\
\hline DN Gem & (1912) & Weak, broad H, He II & (3) (4) \\
\hline DQ Her & (1934) & $\begin{array}{l}\text { Strong, broad H, He II, } \lambda 4650 \text {. } \\
\text { Possibly very weak He I. Also } \\
\text { nebular lines }\end{array}$ & (6) \\
\hline CP Lac & (1936) & Weak $\mathrm{H}$ & (5) \\
\hline DI Lac & (1910) & $\begin{array}{l}\text { Relatively narrow } \mathrm{H}, \mathrm{He} \text { II. Wide, } \\
\text { shallow } \mathrm{H} \text { absorption }\end{array}$ & (2) (3) \\
\hline HR Lyr & (1919) & Weak He II, $\lambda 4650$ & (3) \\
\hline V841 Oph & $(1848)$ & $\begin{array}{l}\text { Moderate H, He II. Weak HeI, } \\
\lambda 4650 \text {. Lines sharp }\end{array}$ & (3) \\
\hline GK Per & $(1901)$ & $\begin{array}{l}\text { Strong broad H, He II. Moderate } \\
\text { He I. Weak Ca II. Absorption spec- } \\
\text { trum of K2IVp. Also nebular lines }\end{array}$ & (2) (3) (48) \\
\hline RR Pic & (1925) & Weak H, Strong He II, moderate & $(2)(3)(48)$ \\
\hline CP Pup & $(1942)$ & Strong H, He II. Weak He I, $\lambda 4650$ & (3) \\
\hline V1059 Sgr & (1898) & Continuous at low dispersion & (4) \\
\hline RW UMi & (1956) & $\mathrm{H}, \mathrm{He} \mathrm{I}, \mathrm{He} \mathrm{II}+$ nebular lines & (7) \\
\hline \multicolumn{4}{|c|}{ (ii) Spectra of recurrent novae at minimum light } \\
\hline $\mathrm{T} \mathrm{CrB}$ & $\begin{array}{l}(1866 \\
1946)\end{array}$ & $\begin{array}{l}\text { Strong H, HeI, CaII. Weak He II. } \\
\text { gM3 absorption. Also nebular } \\
\text { spectrum }\end{array}$ & (8) \\
\hline RS Oph & $\begin{array}{l}(1898, \\
1933, \\
1958, \\
1967)\end{array}$ & $\begin{array}{l}\text { Confused by nebular emission, but } \\
\text { contains absorption lines of late- } \\
\text { type shell spectrum }\end{array}$ & (9) \\
\hline T Pyx & $\begin{array}{l}(1890 \\
1902, \\
1920 \\
1944, \\
1966)\end{array}$ & Strong, sharp H, He II & (3) \\
\hline V1017 Sgr & $\begin{array}{l}(1901 \\
1919 \\
1973)\end{array}$ & $\begin{array}{l}\text { Weak, broad H, He II. Absorption } \\
\text { spectrum of G5III p }\end{array}$ & (2) (5) \\
\hline \multicolumn{4}{|c|}{ (iii) Spectra of dwarf novae at minimum light } \\
\hline RX And* & $\left(14^{d}\right)$ & $\begin{array}{l}\text { Strong, broad H. Moderate He I, } \\
\text { Ca II. Faint He II. }\end{array}$ & (11) \\
\hline AR And & $\left(68^{\mathrm{d}}\right)$ & Strong H & (28) \\
\hline UU Aql & $\left(56^{d}\right)$ & Strong $\mathrm{H}$ & (12) \\
\hline AT Ara & $\left(70^{d}\right)$ & Moderate $\mathrm{H} ; \mathrm{G}$ band absorption? & (55) \\
\hline SS Aur & $\left(54^{d}\right)$ & Broad, strong H, Ca II. Weak He I, Fe II & (11) \\
\hline Z Cam* & $\left(20^{d}\right)$ & $\begin{array}{l}\text { Broad (occasionally double), strong } \mathrm{H} \text {, } \\
\text { He I, Ca II. Weak absorption of G-type } \\
\text { star }\end{array}$ & (11) (13) \\
\hline
\end{tabular}


(iii) Spectra of dwarf novae at minimum light

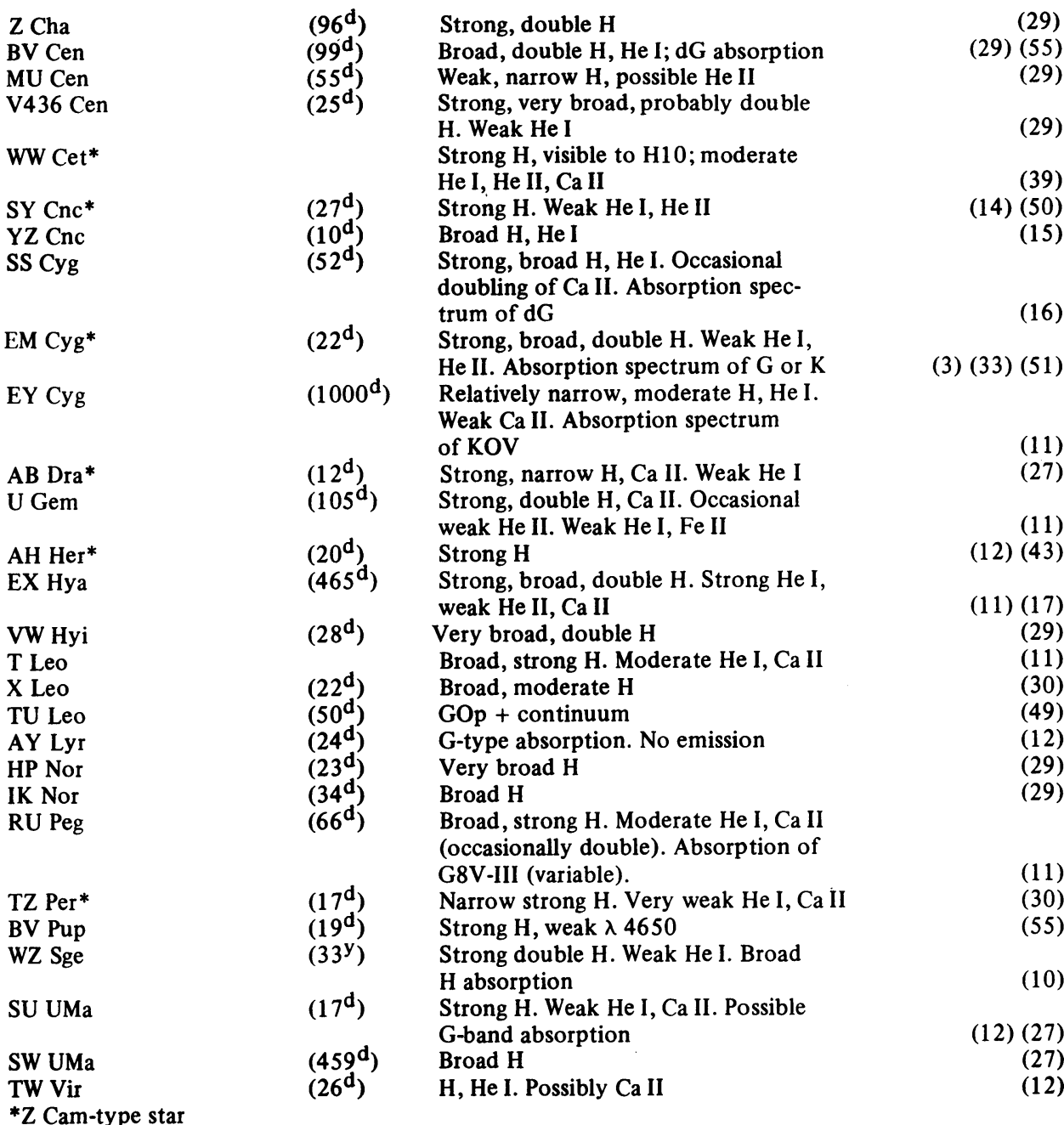

(iv) Spectra of nova-like variables

$\begin{array}{ll}\text { AE Aqr } & \begin{array}{l}\text { Strong H, Ca II. Both Ca II emission } \\ \text { and full absorptionspectrum of dK-type } \\ \text { Weak, broad H. Very weak } \lambda \text { 4650, } \\ \text { He II. Boad H and narrow He I } \\ \text { absorption } \\ \text { He absorption, weak emission } \\ \text { reversals } \\ \text { Weak, broad H } \\ \text { AM CVn }\end{array} \\ \text { Broad H, weak He I. Possible broad } \\ \text { V751 Cyg } & \text { H absorption } \\ \text { MV Lyr } & \text { Strong H, He II. Weak He I } \\ \text { VV Pup } & \end{array}$


(iv) Spectra of nova-like variables

\begin{tabular}{|c|c|c|}
\hline V Sge & $\begin{array}{l}\text { Broad, hazy H, He II, O III, O VI, N IV, } \\
\text { NV }\end{array}$ & (23) \\
\hline CL Sco & Strong $\mathrm{H}$, weak $\mathrm{He}$ I, He II & (12) \\
\hline VY Scl & $\begin{array}{l}\text { Strong H, weak He I, He II. Weak broad } \\
\mathrm{H} \text { absorption }\end{array}$ & (53) (54) \\
\hline VZ Scl & $\begin{array}{l}\text { Broad H, He I, He II. Possibly Ca II } \\
\text { absorption }\end{array}$ & (24) \\
\hline RW Tri & Strong H, He I. Weak He II. & (25) \\
\hline UX UMa & $\begin{array}{l}\text { Strong He II. Weak H. Very weak } \\
\lambda 4650 \text {. Broad, very shallow H } \\
\text { absorption. Possible Ca II absorption }\end{array}$ & (26) \\
\hline AN UMa & Strong $\mathrm{H}, \mathrm{He} \mathrm{I}, \mathrm{He}$ II & (49) \\
\hline$C D-42^{\circ} 14462$ & $\begin{array}{l}\text { Broad } \mathrm{H} \text { absorption with emission re- } \\
\text { versals, He I, } \lambda 4471 \text { absorption }\end{array}$ & (34) (35) \\
\hline $\mathrm{BD}-7^{\circ} 3007$ & $\begin{array}{l}\text { Broad } \mathrm{H}, \mathrm{He} \mathrm{I} \text { and } \mathrm{Ca} \text { II absorption. } \\
\mathrm{H} \text { emission reversals }\end{array}$ & (36) \\
\hline HDE 310376 (QU Car) & $\begin{array}{l}\text { Weak He II, } \lambda 4650 \text {. Weak broad H } \\
\text { absorption }\end{array}$ & (40) (46) \\
\hline Sco $\mathrm{X}-1$ & $\begin{array}{l}\text { Strong H, He II. Weak He I, } \lambda \text { 4650, } \\
\text { Fe II, O II }\end{array}$ & (41) \\
\hline Cyg X-2 & $\begin{array}{l}\text { Strong He II. } \mathrm{H} \text { and } \mathrm{Ca} \text { II absorption }+ \\
\text { dG absorption }\end{array}$ & (42) (52) \\
\hline LS $55^{\circ}-8$ & $\begin{array}{l}\text { Broad H, He II, } \lambda 4650 \text {. Weak broad H } \\
\text { absorption }\end{array}$ & (44) \\
\hline Fiege 24 (EG20) & Sharp, weak $\mathrm{H}, \mathrm{Ca}$ II. Broad $\mathrm{H}$ absorption & (45) \\
\hline
\end{tabular}

\section{References to Table II}

1. C. R. Lynds (private communication)

2. Kraft (1964a)

3. Greenstein (1960)

4. Humason (1938)

5. Kraft (1964b)

6. Greenstein and Kraft (1959)

7. Zwicky (1965)

8. Kraft (1958)

9. Wallerstein (1963)

10. Krzeminski and Smak (1971)

11. Kraft (1962)

12. Elvey and Babcock (1943)

13. Kraft et al. (1969)

14. Herbig (1950)

15. Bretz (1965)

16. Walker and Chincarini (1968)

17. Kraft (1966)

18. Walker (1966)

19. Smak and Stepien (1968)

20. Cowley et al. (1975)

21. Greenstein (1954)

22. Herbig (1960b)

23. Herbig et al. (1965)

24. Greenstein (1966)

25. Walker (1963a)

26. Walker and Herbig (1954)

27. Herbig (1960a)

28. Markaryan (1967)
29. M. W. Feast (private communication)

30. R. P. Kraft (private communication)

31. Greenstein and Matthews (1957)

32. Warner and Robinson (1972a)

33. Robinson (1974)

34. Bond and Landolt (1971)

35. Wegener (1972)

36. Cowley and MacConnell (1972)

37. Herbig and Rao (1972)

38. Robinson et al.

39. Liller (1962)

40. Stephenson et al. (1968)

41. Westphal et al. (1968)

42. Lynds (1967)

43. Robinson (1973b)

44. Greenstein et al. (1970)

45. Eggen and Greenstein (1965)

46. Schild (1969)

47. Stephenson (1967)

48. Gallagher and Oinas (1974)

49. Bond and Tifft (1974)

50. Robinson (1973c)

51. Burbidge and Burbidge (1953)

52. Kraft and Demoulin (1967)

53. Herbig (1964)

54. Burrel and Mould (1973)

55. N. Vogt (private communication) 
timescale (or energy) and the temperature of the emission line region. Comparison with the spectra of nuclei of planetary nebulae, for which Zanstra temperatures are known, suggests that objects showing $\lambda 4650$ emission have $T \sim 35000 \mathrm{~K}$, those showing He II but not $\lambda 4650$ have $T \sim 25000 \mathrm{~K}$ and those showing only $\mathrm{H}$ and HeI have $T \sim 10000$ $20000 \mathrm{~K}$. These results assume normal $\mathrm{He}, \mathrm{C}$ and $\mathrm{N}$ abundance.

There are many examples of long term variability in the spectra. Thus Herbig (Smak and Stepien, 1968) obtained coude spectra at $16 \AA \mathrm{mm}^{-1}$ that showed only Balmer emission in TT Ari in 1961, but a plate at $28 \AA \mathrm{mm}^{-1}$ taken at McDonald Observatory in 1969 has the $\lambda 4650$ group of lines strong enough to show four components, and there is weak $\lambda 4686 \mathrm{He}$ II. This is confirmed by the observations of Cowley et al. (1975).

Changes in the spectrum of UX UMa have been documented by Walker and Herbig (1954). Early spectra taken by Struve (1948), which have been published by Linnell (1949), showed strong broad $\mathrm{H}$ and HeI absorption lines, with only occasional weak Balmer emission. However, the spectra taken by Herbig in 1953 show very prominent bright $\lambda 4686$ as well as Balmer absorption and emission.

Apart from spectrum variations correlated with orbital phase, there are also variations on a shorter time scale than those given above. Thus, in WZ Sge HeI is sometimes present (Kraft, 1966).

Several objects (see Table II) show either permanently doubled emission lines, or occasional double structure of normally broad lines. The width of the lines is usually in the range 200-1500 $\mathrm{km} \mathrm{s}^{-1}$. These line widths were at one time (Greenstein, 1960) thought to indicate high velocity ejection of material as in WR stars. However, the existence of line doubling, and the fact that the emission lines show orbital radial velocity motions, demonstrates that the emission comes from a rapidly rotating region surrounding one of the components of the binary system. This has been confirmed by the presence of a 'rotational disturbance' in the radial velocity curve during partial eclipses of DQ Her (Greenstein and Kraft, 1959). In earlier studies (e.g. Kraft, 1962) it was undecided whether the line doubling might perhaps be produced by self-reversal in optically thick lines. The time resolved spectra, which show $V / R$ intensity variations, certainly favour a geometric explanation for the doubling (see especially the spectra of RW Tri (Walker, 1971) in which the longward component is sometimes absent).

The radial velocity variations of the emission lines are out of phase with the velocity variations of the late-type absorption spectrum (when one is visible). In the case of UX UMa, the broad absorption lines and the $\mathrm{H}$ emissions are in phase (Struve, 1948; Walker and Herbig, 1954). In WZ Sge the doubled emission lines and the H absorption spectrum are both stationary to within observational error (Krzeminski and Kraft, 1964). There is thus no doubt that the rotating region (which we will call a disc) surrounds the hotter member of each binary system; we will always refer to this as the primary, irrespective of whether or not it is the most massive component.

The nebular spectra, visible in some nova remnants, do not show radial velocity variations and are therefore detached from the binary systems. The nebular spectra clearly arise from ejected material, probably decelerated to low velocities by the interstellar medium.

There is no evidence for any systematic difference in gamma velocity for the emission and absorption lines in the double-line binaries, showing that no rapid outflow of the emitting material takes place (Kraft and Luyten, 1965). 
There are many variations in the emission spectra that appear synchronized with the orbital period. In SS Cyg, although no periodic photometric variations have been found, Vojkhanskaya (1973a) has found variations in Balmer emission line strength synchronous with the orbital period. Such variations show that the emission is not uniformly distributed in the disc, and also indicate effects of finite optical thickness in the disc. Here we will draw attention to two observations that indicate that, at least in some objects, the lines requiring high temperatures are located in a restricted region in the disc. Trailed spectra of WZ Sge (Krzeminski and Kraft, 1964) show that the disc contributes very strong, double, stationary $\mathrm{H}$ emission lines. In addition there is a weak $\mathrm{H}$ emission line which varies in radial velocity and forms an S-wave between the stationary lines. Krzeminski and Smak (1971) point out that the weak HeI emission in WZ Sge appears only as an S-wave; there is no stationary double component, showing that the region forming the S-wave emission is only a small part of the disc and is of higher temperature.

He I emission also appears in the spectrum of U Gem (Kraft, 1962, Figure 8), but only during part of the orbital cycle and only as a single line (corresponding to the blueward component) whereas all other lines are clearly double.

Finally, we mention that in RUPeg the luminosity class of the absorption spectrum varies around the orbit, being III when the binary components are in conjunction (red star behind) and $V$ at right angles to this position. The spectral type (temperature class) does not vary appreciably. This can be taken as an indication that the gravitational acceleration varies appreciably over the surface of the secondary.

\section{Photometric Properties at Minimum Light}

Following his discovery of rapid light variations in MV Lyr (三MacRae $+43^{\circ} 1$, Walker, 1954), Walker initiated a program of $U B V$ photometry of cataclysmic variables at minimum light. He found that rapid light variations are a universal feature of these stars (Walker, 1957). We can therefore only determine mean colours at minimum light, the uncertainties in which are typically $\sim 0.1$ mag.

The two-colour diagram for mean colours at minimum light is shown in Figure 1. The data for this diagram have been taken principally from the compilations and observations of Mumford (1967a, 1971). A few of the fainter nova remnants are at distances of $1 \mathrm{kpc}$ or more and may be considerably reddened by interstellar extinction. Also shown is the theoretical black body line (Matthews and Sandage, 1963).

The majority of the cataclysmic variables fall near the blue end of the black body relationship, in a region where quasi-stellar objects are also to be found. However, there are also redder objects as is to be expected from the appearance of late-type absorption spectra in some of these stars (Table I). Perhaps the only anomalous star is AB Dra, which has $B-V=0.96$, but in which no late-type absorption spectrum was detected by Herbig (1960a). This star should be re-investigated at higher spectral resolution.

From the description of the spectra given in Table II it is clear that the colours of the cataclysmic variables are produced by the combination of light emitted from four sources (the primary, the secondary, the emission line region and the continuum region) and as such are of limited interpretative value. Although there is some correlation of $B-V$ with orbital period, arising from the appearance of the secondary spectrum for periods greater than $6 \mathrm{~h}$, the relationship is very loose. Of more interpretative value 


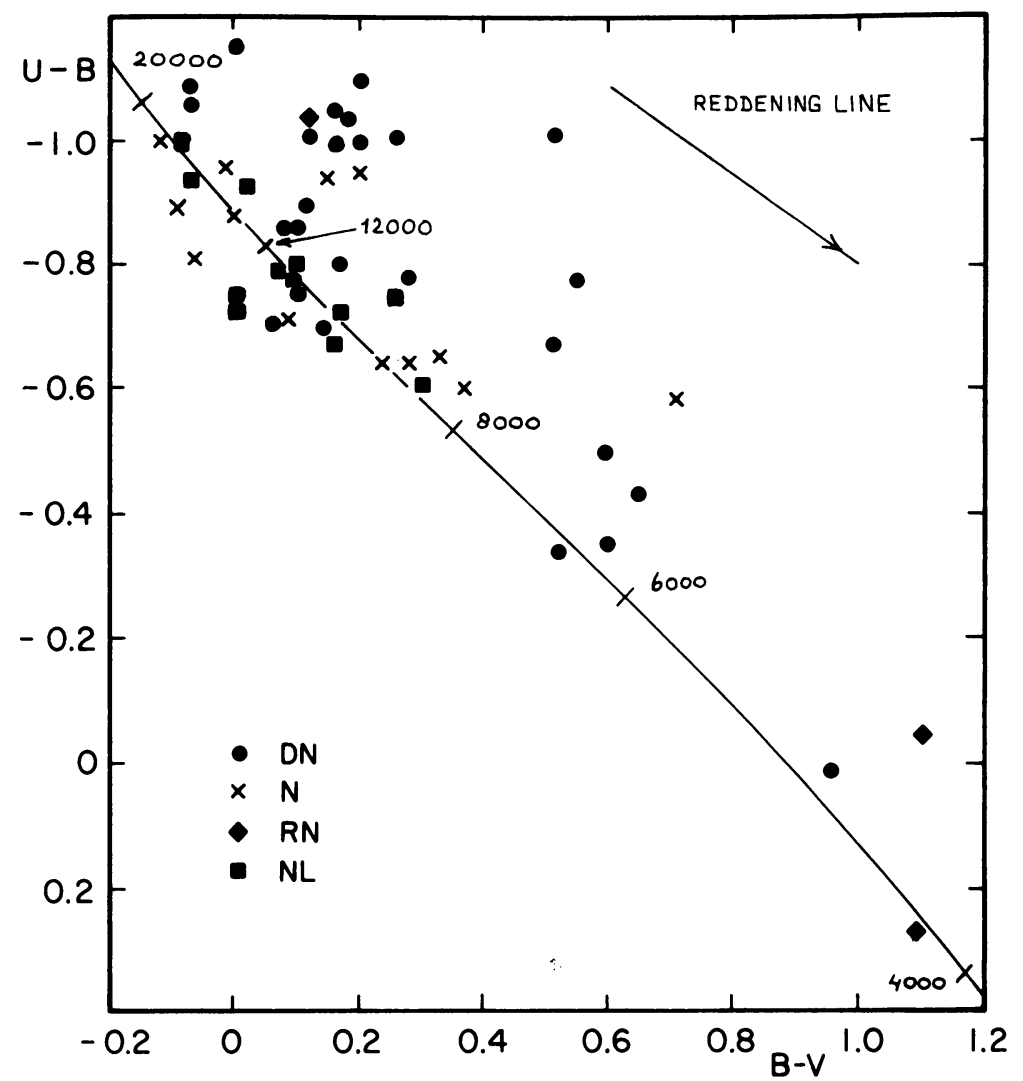

Fig. 1. Two-colour diagram for cataclysmic variables.

are the changes in magnitude and colour around an orbital cycle.

Walker's early work (Walker, 1956, 1958, 1961) which showed that DQ Her is an eclipsing binary, also showed the presence of 'shoulders' in the light curve before and after eclipse. This effect has been seen in a number of objects and is now recognised as a hump in the light curve, usually cut into by an eclipse. In some objects, such as Z Cam (Kraft et al., 1969), TT Ari (Smak and Stepien, 1969) and VW Hyi (Warner, 1975a), not known to eclipse, the periodic occurrence of a hump has led to the determination of the orbital period. In a few objects (e.g. WZ Sge, AM CVn, T CrB) two humps of different width appear in one binary period.

In those systems in which the hump is of considerable amplitude it is found that it lasts for about half of an orbital period. Systems having small amplitude humps probably follow this same rule but the outer portions of the humps are lost in the general flickering or in other, independent small amplitude systematic variations.

In general it is found that the emission line intensities reach maximum near the peak of the hump in the light curve (Krzeminski, 1965; Herbig, 1960b). However, the variations in line intensity are much too small to account for the amplitude seen in the broadband photometry, so we conclude that the hump is principally caused by variations in the source of continuous radiation. 
Along with the hump in the light curve there are colour changes around the orbital cycle. The colour variations of Z Cam (Kraft et al., 1969, Figure 4) are typical; the hump is bluer in $B-V$ and redder in $U-B$ than the colours outside of the hump region.

As in other binary systems, eclipse phenomena have great interpretative potential, but in the cataclysmic variables the complexities are such that we are only just beginning to profit from eclipse observations. The complexities arise from the possible permutations among total and partial eclipses of the primary, the emission disc, and the continuum source. Early observations of DQ Her (Walker, 1956, 1958) showed that it was not possible to get good agreement between theoretical and observed eclipse curves, that there is an asymmetry in the eclipse and that there are cycle-to-cycle variations in the shape and depth of eclipse. The extensive observations of RW Tri (Walker, 1963a) showed even greater variations, with a good correlation between depth of eclipse and brightness outside of eclipse. These and other observations imply that in general the eclipses are of the disc and continuum source, both of which may be variable in extension and brightness.

Pronounced colour changes are detectable during eclipses. In most cases these colour changes are the mirror image of those observed during passage through the hump in the light curve. Together with the fact that eclipses usually occur at or just after the peak of the hump, these colour changes clearly demonstrate that the light source responsible for the hump is being eclipsed. The exceptions to the general behaviour, i.e. those stars in which $U-B$ is redder during eclipse, are UX UMa, WZ Sge and AM CVn. In all these stars, unlike other eclipsing systems, broad absorption lines are visible in their spectra (discussed in detail later). Thus in these cases we see that the absorption line source is brighter (and, according to spectra taken through eclipse, remains uneclipsed) and redder than the continuum source.

It is appropriate at this point to mention some other spectroscopic results obtained during eclipses. Time-resolved spectra of RW Tri (Walker, 1971), DQ Her (Greenstein and Kraft, 1959), U Gem (Kraft, 1962), WZ Sge (Krzeminski and Kraft, 1964), and UX UMa (Walker and Herbig, 1954) all show that the Balmer emission lines persist through eclipse, although usually weakened. The Balmer decrement is steeper during eclipse and $\mathrm{He}$ II $\lambda 4686$ is greatly weakened in DQ Her (where, however, nebular emission contaminates the spectrum), whereas the Balmer decrement is less steep and $\lambda 4686$ is almost unchanged in UX UMa.

From these results it is clear that the eclipses must be due primarily to loss of light from the continuum, the principal emission line source being only partially eclipsed. From the change in Balmer decrement and the selective weakening of $\lambda 4686$ it is again apparent that there are variations in temperature in the disc. The colour changes during eclipse thus reflect the removal of the continuum leaving an almost pure emission line source. In some cases the secondary may contribute significantly during eclipse, but there is no instance of the secondary spectrum being detected only in eclipse.

As we remarked earlier, flickering is a ubiquitous feature of cataclysmic variables at minimum light. The earlier work by Walker, Mumford, Smak, Krzeminski and co-workers mostly used conventional d.c. amplifier and chart recorder techniques which limited detailed observations of the flickering to timescales of about a minute and longer. In addition, because of the necessity to check frequently sky brightness, comparison star, dark current and guiding, the records tended to be broken into short pieces which obscured some important properties of the flickering activity. Some progress has been 
made, by the writer and his colleagues over the last few years, towards a more detailed study of the flickering. The observing technique, using pulse counting photometry and minicomputers has been described elsewhere (Nather and Warner, 1971; Warner, 1972a). This technique provides long sections of light curves of CV's with time resolution down to $1 \mathrm{~s}$ and thus is ideal for studying rapid brightness variations. Initially this research was undertaken in the hope of studying conditions in white dwarf atmospheres, the belief at that time being that the flickering is caused by infall of matter onto the primaries of the cataclysmic variables.

First results on objects such as GK Per, DQ Her, MV Lyr, T CrB, CZ Ori, SS Cyg, etc. (this material is still mostly unpublished) confirmed Walker's conclusion that all CV's vary on timescales of minutes and showed in addition that most objects have variations on timescales of seconds. It was furthermore found that the nature of the variations is different from one object to another; thus MV Lyr shows continuous very rapid flickering, whereas GK Per is more leisurely in its activity. A study is underway to describe quantitatively these flickering properties, with the aim of looking for correlations with other properties, e.g. orbital period.

In those systems (e.g. U Gem, VV Pup, VW Hyi) that have a large amplitude hump, flickering shows a concomitant increase in amplitude at the top of the hump. This very clearly demonsirates that the flickering is closely associated with the same source of continuum radiation that produces the hump itself. As far as the slower (timescales of minutes) flickering is concerned, this has been confirmed by Walker's (1966) timeresolved electronographic spectra of SSCyg, MV Lyr and T CrB, in which the emission line intensities remained constant while the continuum brightness changed. The persistence of the flickering (at reduced amplitude) for the half period outside the hump shows that the continuum source is not entirely obscured at any time. Either it is partially covered by an optically thick region, or it is partially visible through a region of finite optical thickness. If we make the reasonable assumption that the average flickering amplitude remains a constant fraction of the light from the continuum source, we can deduce the fraction of light contributed to the system by measuring the apparent change in flickering amplitude around the orbital cycle. This has been done for U Gem by Harwood (1973), using the photometry reported by Warner and Nather (1971). It was found that the continuum source contributes just over half of the light in the system in white light) at phase 0.5 (i.e. away from the hump). This is about the same light level as the bottom of the eclipse.

Walker's photometric studies have shown that the flickering amplitude is greatest in the ultraviolet region, in accordance with our deduction that the continuum (which dominates at the shorter wavelengths) is the source. Spectrophotometric study of the flickering by Walker and Chincarini (1968) showed that in SS Cyg the colour temperature is increased at the maximum of a short-lived brightness fluctuation.

Eclipses of U Gem, RW Tri and UX UMa have been observed with high time resolution (Warner and Nather, 1971, 1972b). In all cases it is found that the rapid flickering is greatly reduced, or is essentially absent. The best example of this is seen in U Gem in Figure 2; in the centre of eclipse the light level has fallen by about a factor of 2 , but the flickering has been reduced by at least a factor of 10: the width of the data band is principally due to scintillation and photon counting noise. The irregular grouping of the data points during ingress gives the impression that the flickering amplitude is present at the beginning but is rapidly reduced. 
B. WARNER

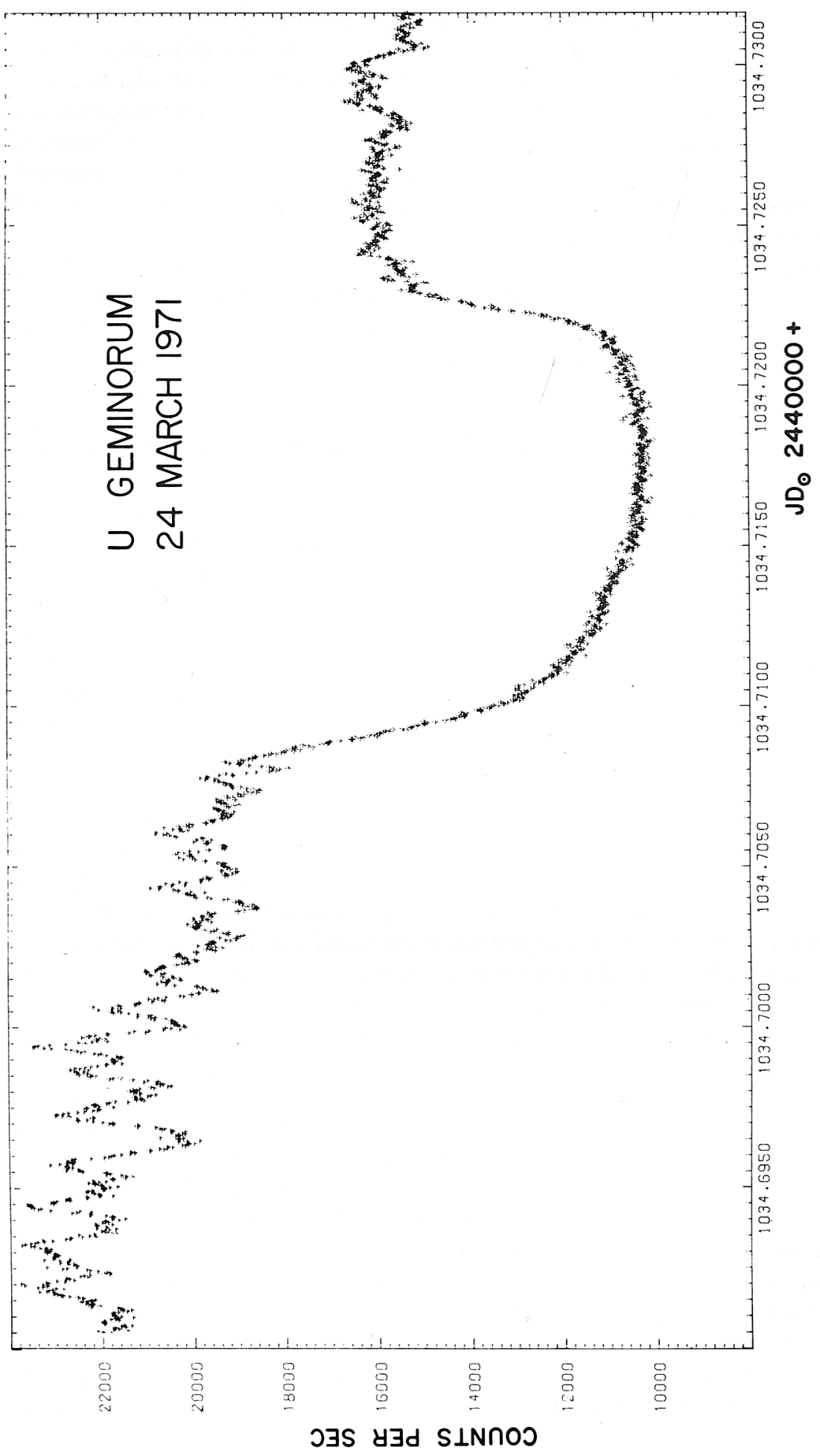

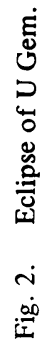


The disappearance of flickering during eclipses of U Gem, UX Uma and RW Tri is in complete agreement with the spectroscopic observations that in these objects eclipse is of the continuum source. This is a valuable conclusion because it means that we can estimate the size of the continuum source from durations of ingress and egress.

The marked asymmetry of ingress and egress in Figure 2 is characteristic of the eclipsing CV's. This, and the unstable shape and timing of the eclipses (e.g. DQ Her, Walker, 1956), shows that in general we are not seeing the eclipse of a normal star, i.e. the continuum source is able independently to vary its brightness and position.

The information and deductions we have given so far are sufficient to provide the basis of a general working model. The similar behaviour of so many CV's indicates that this model should be applicable to all.

\section{The Model}

The basic model for CV's was first proposed by Crawford and Kraft (1956) and is an extension of the ideas introduced in Kuiper's (1941) $\beta$ Lyr model. The essential feature is the recognition that the disc of material around the primary is more likely to have been generated by matter transferred from the secondary than from matter ejected from the primary itself. Consequently, it is necessary to identify a mechanism that can stimulate mass loss from the secondary; the most probable being that the secondary is a "contact component' (Kopal, 1955), i.e. it fills its Roche lobe. Evaporation of the atmosphere of the secondary can then take place in the neighbourhood of the inner Lagrangian point. A general review of the evolution of systems containing contact components has been given by Paczynski (1971). Paczynski and Sienkiewicz (1972) show that the rate of mass loss from a contact component is sensitively dependent on the amount by which its radius exceeds that of the Roche lobe. We conclude that the secondaries of CV's cannot overfill their Roche lobes by a significant amount.

The incompleteness of the model was clearly demonstrated by Walker and Herbig's (1954) discussion of UX UMa. In order to account for the photometric hump and the variation of emission line intensities they introduced a bright region, fixed with respect to the line of centres of the two component stars. Krzeminski (1965) placed the continuum hot spot in U Gem on the following hemisphere of the primary, but maintained the asymmetrical distribution of emission line intensities in the disc. Objections to this, and other features of Krzeminski's U Gem model, were raised by Warner and Nather (1971) and Smak (1971a). Both independently proposed a more complete and, at least as far as the more gross features are concerned, a physically reasonable model incorporating the features outlined in the two previous sections. The continuum source is seen as a hot spot produced by a stream of gas leaving the inner Lagrangian point and impacting onto the outer edge of the disc. The kinetic energy of impact maintains the brightness of the spot; variations in the rate of mass transfer allow the general brightness of the spot to vary on a number of timescales, from years (as in VV Pup - Smak, 1971b) to seconds (in which the flickering activity is supposed to arise from inhomogeneities in the interstellar stream). The asymmetry of eclipse light curves is a consequence of the different amount of foreshortening of the bright spot at ingress and egress. The asymmetry in the emission line distribution arises from the excess emission produced near the bright spot; lines such as He I in WZ Sge and U Gem are produced only in the vicinity of the bright spot. 
For such a model, there are a number of quantitative relationships that we can immediately write down. Kepler's law gives

$$
4 \pi^{2} a^{3}=G\left(M_{1}+M_{2}\right) P^{2}
$$

where $a$ is the separation between centres of the stars and $P$ is the orbital period. Paczynski (1971) shows that the radius of a spherical star having the same volume as that of the Roche lobe can be written (correct to $\sim 2 \%$ ) as

$$
\frac{R_{2}}{a}=0.38+0.20 \log q \text { for } 0.5<q<20
$$

or

$$
\frac{R_{2}}{a}=0.462\left(\frac{q}{1+q}\right)^{\frac{1}{3}} \quad \text { for } \quad 0<q<0.5
$$

where $q=M_{2} / M_{1}$.

Equations (1) and (2b) give a formula for the mean density of the secondary:

$$
\bar{\rho}=\frac{M_{2}}{\frac{4}{3} \pi R_{2}^{3}}=\frac{3 \pi}{0.462^{3} G} P^{-2}=1.43 \times 10^{9} P\left(\mathrm{~s}^{-2} \mathrm{gm} \mathrm{cm}^{-3}\right.
$$

(Faulkner et al., 1972).

The existence of a unique relationship (for given chemical composition) between $\bar{\rho}$ and $M_{2}$ for main sequence stars implies through Equation (3) that there should be a $P-M_{2}$ relationship, and hence a $P-L_{2}$ (period-luminosity of secondary) relationship. We show in Section 6 that this relationship quantitatively explains the appearance of the secondary spectra in systems with $P \widetilde{>} 6^{\mathrm{h}}$. For the present, we note that $\bar{\rho}=120 \mathrm{gm} \mathrm{cm}^{-3}$ for a star with $M_{2}=0.085 M_{\odot}$ (Grossman et al., 1971) which is the minimum mass for a hydrogen-burning dwarf (Graboske and Grossman, 1971). Therefore, for $P \geqslant 0.96 \mathrm{~h}$ the secondary can exist as a normal main sequence star. For shorter orbital periods the secondary must be made of helium, be a non nuclear burning dwarf, or be degenerate. Also, for secondaries of spectral type later than F0, systems with $P>10 \mathrm{~h}$ must contain evolved secondaries.

The stellar models for the lower main sequence calculated by Copeland et al. (1970), Grossman et al. (1971), and R. L. Wagner and R. Loren of the University of Texas, together with the observed masses and radii (Warner, 1973b, Table II) show that

$$
\frac{R_{2}}{R_{\odot}}=0.959 \frac{M_{2}}{M_{\odot}}
$$

to reasonable accuracy in the range $0.2 \leqslant M_{2} / M_{\odot} \leqslant 1.5$, and this same relationship is probably adequate down to $0.085 M_{\odot}$. Equation (3) then yields

$$
\frac{M_{2}}{M_{\odot}}=3.18 \times 10^{-5} P(\mathrm{~s})
$$

as the mass-period relationship (for $0<q<0.8$ ). The form of Equation (5) was first derived by Faulkner (1971); the numerical factor, and the independence from total mass, both rely on being able to represent the lower main sequence by Equation (4). Eventually 
we may hope to use a purely empirical $R_{2}-M_{2}$ relationship, so avoiding the uncertainties still inherent to stellar models.

From (4) and (5) we also have the $P-R_{2}$ relationship:

$$
\frac{R_{2}}{R_{\odot}}=3.04 \times 10^{-5} P(\mathrm{~s})
$$

Equation (6) has an interesting implication for the rotational velocity of the secondary. For synchronously rotating secondaries, the equatorial velocity is $\nu_{2}=2 \pi R_{2} / P=128$ $\mathrm{km} \mathrm{s}^{-1}$ from (6). The total line width observed in those objects showing secondary spectra should therefore be $\sim 256 \sin i \mathrm{~km} \mathrm{~s}^{-1}$ independent of $P$. A comparison with observations is made in Section 7.

A rough estimate of the size of the disc can be made by appealing to single particle trajectories. Prendergast (1960) has shown that even with a hydrodynamical treatment of motions in the disc, the single particle energy equation relating the orbital velocity $v(r)$, mass $M_{1}$ and radius vector $r$ in the disc is a good approximation, i.e.

$$
r v^{2}(r)=G M_{1}
$$

Because material is being delivered at the outer edge of the disc, via the inter-star stream, with a fairly well-defined angular momentum, we may expect that a considerable amount of mass will orbit the primary at a radius $r_{d}$ having the same angular momentum as the arriving material. Assuming for the moment that angular momentum is conserved about $M_{1}$, we have

$$
v\left(r_{d}\right) r_{d}=2 \pi f^{2} a^{2} P^{-1}
$$

where $f$ is the distance from the centre of the primary to the inner Lagrangian point (in units of $a$ ). Equations (1), (7) and (9) yield

$$
\frac{r_{d}}{a}=f^{4}(1+q)
$$

A convenient formula for $f$, deduced from the tabulations of Plavec and Kratochvil (1964) and accurate to one percent, is

$$
f=0.500-0.227 \log q \quad 0.1 \leqslant q \leqslant 10
$$

Comparison with the accurate calculations of Flannery (1974) shows that (due to angular momentum exchange with the secondary) Equation (9) overestimates $r_{d}$ by $\sim 30 \%$. This is in fact not inconvenient, because the redistribution of angular momentum within the gaseous disc is likely to increase its radius by at least this amount. Calculations by Piotrowski and Ziolkowski (1970) of the maximum dimensions of stable periodic orbits in close binaries give radii roughly twice those found from Equation (9).

The semi-amplitude $K_{1}$ of the primary's radial velocity curve is

$$
K_{1}=\frac{2 \pi q a \sin i}{P(1+q)}
$$

hence from (8), (9) and (10) we have

$$
\frac{K_{1}}{v\left(r_{d}\right) \sin i}=f^{2} q
$$


$K_{1} / v\left(r_{d}\right) \sin i$ is thus seen to be a function only of $q$. Identifying $v\left(r_{d}\right) \sin i$ as the halfwidth of the observed emission lines from the disc, we can compare (11) with observations for those systems (not only CV's) that both possess discs and are double line binaries. This has been done by Kruszewski (1967), Warner (1973b) and Piotrowski (1975). The agreement is excellent and provides a means of estimating $q$ for those systems for which $K_{1} / v\left(r_{d}\right) \sin i$ can be observed.

The position of the hot spot depends on the trajectory of the inter-star stream (which is governed principally by gravitational forces) and the radius of the disc at which impact occurs. For the simple assumptions used above, Warner and Peters (1972) made calculations, corrected by Flannery (1975), which provide angles and relative velocities of impact. Observationally, the hot spot may be located from the phase shift between the position of maximum light (corresponding to that time when the bright spot is most nearly face-on) and time of mid-eclipse (when the bright spot - or possibly the primary is eclipsed). From photometric observations it is often possible to provide only combinations of various angles but these may be useful in setting limits on $q$ (Warner and Peters, 1972).

The various observed forms of light curve result naturally from the model by the range of inclinations and of $q$. Lin (1975) has discussed quantitatively the information that can be derived from light curves of CV's.

The size of the bright spot can be estimated observationally from the duration of the hump and from eclipse ingress or egress durations. Longitudinal extensions $\delta$ around the disc of $5^{\circ}$ for U Gem (Warner and Nather, 1971), $7.5^{\circ}$ in VV Pup (Warner and Nather, $1972 a$ ) and $\sim 20^{\circ}$ in Z Cha (Warner, 1974a) have been found. In most other systems with well-defined humps we find $\delta<10^{\circ}$. The diameter of the hot spot is then

$$
l=\frac{\delta}{360} 2 \pi r_{d} \sim 0.017 \delta f^{4}(1+q) a
$$

from (19). This gives $l \sim 2 \times 10^{9} \mathrm{~cm}$ in general.

Throughout the remainder of this review we will have occasion to refer to the primary as a white dwarf. The evidence for a white dwarf primary was originally based on the presence of a hot continuum in stars of low luminosity (Section 6), evidence which must be relegated because the bright spot is in general the dominant light source, and on the presence of apparent extreme pressure-broadening in the absorption spectra of WZ Sge and DI Lac, evidence which we dispute in Section 7. However, the emission lines with wings out to several thousand $\mathrm{km} \mathrm{s}^{-1}$ (Section 7) and the existence of brightness oscillations with periods $\sim 20$ s (Section 12) both indicate objects of the size of white dwarfs.

\section{Masses}

The pre-requisite for mass determination - an eclipsing double line binary - is fully satisfied in only one CV: the dwarf nova EM Cyg provides $M_{1}=0.70 \pm 0.18 M_{\odot}$ and $M_{2}$ $=0.90 \pm 0.17 M_{\odot}$ (Robinson, 1974). However, in two other cases the orbital inclination may be estimated well enough to provide masses. The presence of a single hump per period, but absence of definite eclipses, in Z Cam (Kraft et al., 1969; Robinson, 1973a,d) suggests $i \sim 60^{\circ} \pm 8^{\circ}$ from which $M_{1}=0.97 \pm 0.20 M_{\odot}$ and $M_{2}=0.69 \pm 0.12 M_{\odot}$. In the case of T CrB, Paczynski (1965a) has reinterpreted the radial velocity data of Kraft 
(1958) for an assumed orbital inclination of $68^{\circ}$, based on absence of eclipses. Recently, however, $\mathrm{T} \mathrm{CrB}$ has been found to show a double-humped light curve with an amplitude $\Delta m_{B} \sim 0.4 \mathrm{mag}$ (Bailey, 1975). Because of the domination of the light of T CrB by the $M$ giant, such a double-humped light curve could arise from eclipses of the hot spot (or primary) by the secondary, combined with partial eclipses of the secondary by the disc. The inclination may therefore be much closer to $90^{\circ}$ than was assumed by Paczynski; this leads to masses $M_{1}=1.6 \pm 0.4 M_{\odot}$ and $M_{2}=2.1 \pm 0.5 M_{\odot}$. These are rather uncertain because of the small radial velocity amplitudes in this long period system.

There are, however, problems concerning the interpretation of the velocity amplitudes $K_{1}$ of the primaries. The concentration of line emission in the vicinity of the hot spot, through which the material of the disc is rotating, results in a distortion of the emission line profiles. The observed radial velocity is then a weighted vector sum of the orbital and disc rotational velocities. Smak (1970) has computed corrections to $K_{1}$ for simple models of the distribution of line emission over the disc. Changes in $K_{1}$ of $50 \%$ or more are possible, which may significantly affect the derived mass. Smak gives the example of Z Cam, for which the observed $K_{1}$ is $144 \mathrm{~km} \mathrm{~s}^{-1}$, but freed of distortion it becomes $K_{1} \sim 200 \mathrm{~km} \mathrm{~s}^{-1}$. However, Robinson's (1973d) measurements on the wings of $\mathrm{H} \alpha$ in $\mathrm{Z}$ Cam give $K_{1}=137 \mathrm{~km} \mathrm{~s}^{-1}$, and as the wings are some $100 \mathrm{~km} \mathrm{~s}^{-1}$ from the line centre they are probably formed in the inner parts of the disc away from the bright spot. The conventional method of measuring radial velocities from the centres of gravity of the lines (or the average position of peaks for doubled emission lines) certainly must introduce distortion into the radial velocity curve, producing spurious eccentricities and phase lags between true and apparent conjunction in many cases. As examples we may cite UGem, for which the large $K_{1}$ is thought to arise from distortion (Harwood, 1973) and $\mathrm{VZ} \mathrm{Scl,} \mathrm{in} \mathrm{which} \mathrm{the} \mathrm{effect} \mathrm{is} \mathrm{so} \mathrm{large} \mathrm{that} \mathrm{apparent} \mathrm{maximum} \mathrm{radial} \mathrm{velocity} \mathrm{occurs}$ near eclipse and an eccentric orbit could be deduced. For this star it is shown (Warner and Thackeray, 1975) that these distortions are reconcilable with a normal circular orbit by use of Smak's analysis, but the final data are very uncertain.

A previous estimate $M_{1}=0.12 M_{\odot}$ for DQ Her (Kraft, 1963), based on interpretation of the 71-s oscillations as radial oscillations, can now be discarded as the oscillations are now considered to arise from other processes (Section 12).

In order to obtain a more comprehensive view of masses we use the approach based on lobe-filling secondaries given in the previous section. From (1) and (2) we find

$$
\begin{aligned}
\left(\frac{R_{2}}{R_{\odot}}\right)^{3}\left(\frac{M_{\odot}}{M_{2}}\right) & =1.00 \times 10^{-8} P^{2}\left(\frac{1+q}{q}\right) \frac{R_{2}^{3}}{a} \\
& =1.00 \times 10^{-8} P^{2} F(q)
\end{aligned}
$$

where $F(q)$ is to be evaluated with the aid of Equation (2a) or (2b). Equation (12) is almost independent of $q$ : for $q<0.5$ we have $F(q)=0.462^{3}=0.0986$; from $q=0.5$ to $q=1.5 F(q)$ increases up to 0.119 . Therefore almost any estimate of $q$ is sufficient for us to determine $R_{2}^{3} M_{2}^{1}$ with considerable confidence. In practice we may find $q$ from the value of $K_{1} / v\left(r_{d}\right) \sin i$ as shown in the previous section.

For a given $M_{2}-R_{2}$ relationship for lower main sequence stars we can therefore find $M_{2}$ for essentially all CV's for which $P$ is known. Results are given in Table III. We have used the calculations by Copeland et al. (1970) and Grossman et al. (1971) to define 
$M_{2}\left(R_{2}\right)$. This is slightly different from that used previously (Warner, 1973b) and results in somewhat lower masses.

To deduce $M_{1}$ we require $q$. Table III lists values given by the use of $K_{1} / v\left(r_{d}\right) \sin i$ and also $\left(q^{1}\right)$ from double line binaries. Considering the problems of interpretation, the agreement between $q$ and $q^{1}$ is not too bad. Agreement with the independently derived masses of EM Cyg and Z Cam is also satisfactory. Omitting the two discrepant and uncertain cases of U Gem and VZ Scl we find a mean mass $\left\langle M_{1}\right\rangle=0.94 M_{\odot}$ from the ten stars with $P>10^{4} \mathrm{~s}$. The difference between this and the value $\left\langle M_{1}\right\rangle=1.1 M_{\odot}$ given previously (Warner, 1973b) is an indication of how sensitive the method is to the $M_{2}-R_{2}$ relationship adopted for the main sequence stars. U Gem has been studied in detail by Smak (1971a) and Harwood (1973) and appears to have $M_{1} \sim 0.6 M_{\odot}$, i.e. smaller than the other objects.

From Equations (1), (5) and (10) we find

$$
\sin i=3.35 \times 10^{-8} K_{1}\left(1+q^{-1}\right)^{\frac{2}{3}}
$$

which provides a means of estimating the orbital inclination for those stars with measured $K_{1}$; this method is insensitive to $q$ for $q>0.5$ and can be used as an independent test of our whole approach. Results, given in Table III, are in remarkable agreement with independently estimated inclinations; note in particular that those objects with predicted $i>60^{\circ}$ are eclipsing systems, as expected from the geometry of bright spots in semidetached systems. Equation (13) gives imaginary values of $i$ for U Gem and VZ Scl, again emphasising the probable large distortion of their $K_{1}$; in fact, using $q=0.7$ and $i=65^{\circ}$ for U Gem predicts $K_{1}=150 \mathrm{~km} \mathrm{~s}^{-1}$ from (13).

Omitting the two uncertain cases, the correlation between $P$ and $q$ found by Warner (1973b) is evident in Table III. This is a result of the small range of $M_{1}$. As a result of this, CV's with $P \approx 10^{4}$ s have $K_{1}$ too small to be detectable by present techniques.

A star that apparently does not fit the $P-q$ relationship is GK Per, which has $P=5.9 \times 10^{4} \mathrm{~s}$ and $q=0.24$ according to Paczynski (1965a). The spectral type of the companion is K2IV (Gallagher and Oinas, 1974) with an uncertainty of several subclasses. Equation (3) gives $\bar{\rho}=0.41 \mathrm{gm} \mathrm{cm}^{-3}$, whereas a $G$ or $K$ main sequence star has $\bar{\rho} \sim 2$ $\mathrm{gm} \mathrm{cm}^{-3}$ (Allen, 1963). The secondary of GK Per is therefore in an evolved state, in agreement with its luminosity class. Thus, whereas all the CV's with main sequence secondaries appear to follow a $P-q$ relationship, the one evolved secondary has far too small a mass, suggesting a substantial amount of mass lost. Evolutionary considerations require $M_{2} \geqslant 1 M_{\odot}$ for expansion to have occurred, and the core mass will have been $\sim 0.20 M_{\odot}$. According to Paczynski's value of $q$, the secondary in GK Per will therefore have been stripped almost to its helium core.

\section{Luminosities and Temperatures}

\subsection{MINIMUM LIGHT}

Payne-Gaposchkin (1957), using the absolute magnitude-rate of decline relationship for novae, combined with apparent magnitudes of nova remnants, derived $M_{v}=+4.0$ for fast novae and $M_{v}=+4.7$ for slow novae at minimum light. A later study by Schmidt-Kaler (1962), using additional data, gave a mean luminosity of $M_{v}=+3$. Luyten and Hughes 
TABLE III

Masses of cataclysmic variable stars

\begin{tabular}{|c|c|c|c|c|c|c|c|c|}
\hline & $P\left(10^{4} s\right)$ & $\begin{array}{l}K_{1} \\
\left(\mathrm{~km} \mathrm{~s}^{-1}\right)\end{array}$ & $\begin{array}{l}\nu \sin i \\
\left(\mathrm{~km} \mathrm{~s}^{-1}\right)\end{array}$ & $\mathbf{q}$ & $q^{1}$ & $i^{0}$ & $M_{2} / M_{\odot}$ & $M_{1} / M_{\odot}$ \\
\hline AE Aqr & 3.56 & 135 & 550 & 0.94 & 0.85 & 47 & 1.18 & 1.25 \\
\hline RU Peg & 3.20 & 137 & 500 & 1.15 & 1.19 & 44 & 1.12 & 0.97 \\
\hline EM Cyg & 2.52 & 189 & & & 1.29 & 68 & 0.86 & 0.67 \\
\hline Z Cam & 2.50 & 144 & 700 & 0.73 & 0.74 & 59 & 0.86 & 1.17 \\
\hline SS Cyg & 2.39 & 122 & 500 & 0.86 & 1.06 & 43 & 0.83 & 0.97 \\
\hline RW Tri & 2.00 & & 600 & & & & 0.71 & \\
\hline RX And & 1.83 & 77 & 410: & 0.64 & & 29 & 0.65 & 1.02 \\
\hline T Aur & 1.76 & & & & & & 0.63 & \\
\hline UX UMa & 1.70 & & & & & & 0.61 & \\
\hline DQ Her & 1.67 & 150 & 680 & 0.72 & & 64 & 0.60 & 0.83 \\
\hline SS Aur & 1.56 & 85 & 450: & 0.64 & & 32 & 0.57 & 0.89 \\
\hline U Gem & 1.50 & 265 & 850 & 1.50: & & - & 0.53 & 0.35 : \\
\hline WW Cet & 1.38 & & & & & & 0.50 & \\
\hline RR Pic & 1.25 & & & & & & 0.44 & \\
\hline $\mathrm{VZ} \mathrm{Scl}$ & 1.25 & $275:^{a}$ & 900 & 1.38: & & - & 0.44 & 0.32 : \\
\hline V603 Aql & 1.19 & 37 & 240 & 0.46 & & 15 & 0.40 & 0.87 \\
\hline TT Ari & 1.15 & $55^{\mathrm{a}}$ & 350 & 0.48 & & 23 & 0.38 & 0.79 \\
\hline VW Hyi & 0.642 & & & & & & 0.17 & \\
\hline $\mathrm{ZCha}$ & 0.642 & & & & & & 0.17 & \\
\hline VV Pup & 0.600 & & & & & & 0.16 & \\
\hline EX Hya & 0.594 & $\leqslant 50$ & 700 & $<0.15$ & & & 0.16 & $>1.07$ \\
\hline WZ Sge & 0.492 & $\leqslant 38$ & 860 & $<0.076$ & 0.071 & & 0.12 & $>1.5$ \\
\hline
\end{tabular}

a corrected for distortion

(1965) find $M_{v} \sim+4$ from proper motions of 13 nova remnants. $M_{v}$ estimates based on strengths of interstellar lines and expansion parallaxes are in good accord with these estimates (McLaughlin, 1960).

From a variety of methods, all based on rather few observations, Schmidt-Kaler found $M_{v}=+7.2$ for dwarf novae. A more complete discussion, based on proper notions of 25 stars and radial velocities of 11 stars, allowed Kraft and Luyten (1965) to determine $M_{v}=+7.5$ for the dwarf novae. However, it was pointed out that evidence exists for a substantial spread in luminosity for the dwarf novae. Thus the orbital period-luminosity relationship for secondaries, discussed later in this section, implies that the longer period objects (showing $d G$ spectra - often partially veiled by the continuum) have $M_{v}<6$. Vojkhanskaya (1973b) has also analysed proper motions and radial velocities and finds $M_{v}=7.5$. The dwarf nova BX Pup is probably a member of NGC 2482 and consequently has $M_{v}=6.5$ (Moffat and Vogt, 1975). SS Aur is a member of the Hyades group and RUPeg has a cpm companion which give $M_{v}=6.3$ and 6.2 respectively (Eggen 1967, 1969).

SS Cyg has been observed at minimum light by OAO-2 (Holm and Gallagher, 1974) down to $1500 \AA$. The observations have a large uncertainty (because of the contamination from other stars) but show that the ultraviolet continuum continues to rise down to the shortest wavelengths, resembling a black body of temperature $22000 \mathrm{~K}( \pm 5000 \mathrm{~K})$. More accurate observations of dwarf novae at minimum are urgently required; the bolometric magnitude of these objects is essentially unknown. The best that we can do at 
present is to adopt a bolometric correction of $2 \mathrm{mag}$ (based on the $22000 \mathrm{~K}$ effective temperature) to get $M_{\text {bol }} \sim 5.5$ and $L \sim 0.4 L_{0}$ for dwarf novae at minimum.

The nova remnants RR Pic and V603 Aql have also been observed from OAO-2 (Gallagher and Holm, 1974). Effective temperatures respectively of $\sim 35000$ and $25000 \mathrm{~K}$ were found from observations between $5500 \AA$ and $1500 \AA$. Integrating the flux gives lower limits of $\sim 10 L_{\odot}$ for the bolometric luminosity of the two stars.

We see that both the visual absolute magnitudes and the bolometric luminosities show that the classical nova remnants have on average at least 10 times greater luminosity than the dwarf novae.

\subsection{RECURRENT NOVAE}

The light curves of recurrent novae during outburst resemble those of ordinary novae. Their amplitudes of outburst, however, range from 4 to $9 \mathrm{mag}$, as opposed to the 8-15 mag of classical novae.

Application of the $M_{\nu}$-rate of decline relationship (Payne-Gaposchkin, 1957, Table 1.18) leads to absolute magnitudes $M_{v}(d)$ at minimum light lying in the range -0.8 to 0.8 . These are considerably brighter than the average for classical novae and may be thought to invalidate this way of estimating the absolute magnitudes. There is, however, independent evidence for these results. We will discuss the stars for which sufficient observations are available.

$T C r B$. Both the 1866 and 1946 eruptions had a range of 8.5 magnitudes, leading to $M_{v}(d)=0.1$. The spectrum of $\mathrm{T} \mathrm{CrB}$ at minimum shows $\mathrm{TiO}$ bands corresponding to a gM3 star (Kraft, 1958). In the visible the spectrum is dominated by the $\mathbf{M}$ giant, so $M_{v} \sim-0.4$ (Blaauw, 1963), in agreement with $M_{v}(d)$. Infrared magnitudes (Feast and Glass, 1974) confirm the gM3 classification.

$V 1017$ Sgr. The 1919 eruption had a range of $7.2 \mathrm{mag}$, giving $M_{\nu}(d)=0.8$. V1017 Sgr at minimum shows the presence of a G5III star (Kraft, 1964a) which would have $M_{v} \sim 0.4$ (Blaauw, 1963), again in agreement with $M_{v}(d)$. Feast and Glass (1974) find infrared colours in accord with the spectral type.

$R S$ Oph. The average range of the four outbursts is $7.2 \mathrm{mag}$, giving $M_{v}(d)=-1.1$. The spectrum of RS Oph is complex and the absorption lines occasionally observed probably come from a thick circumstellar shell (Wallerstein, 1963). Feast and Glass find $J-K$ $=1.12$ and $K-L=0.37$ which would suggest a spectral type near M6 (Johnson, 1966). Interpreted as a giant, the infrared colours thus imply $M_{v} \sim-0.5$.

T Pyx. Spectra of T Pyx have been discussed by Catchpole (1969). No spectrum having reasonable resolution has been obtained of T Pyx at minimum. During outburst, Catchpole detected the interstellar $\mathrm{K}$ line with an equivalent width of $0.40 \AA$. From the calibration by Beals and Oke (1953), Catchpole finds that this $\mathrm{K}$ line strength corresponds to a distance of $1050 \mathrm{pc}$. However, T Pyx lies near a node in the galactic rotation curve, and the calibration given by Münch (1968) for such stars shows that a star with $0.4 \AA \mathrm{K}$ line will be at least $2 \mathrm{kpc}$ distant. Adopting $d=2 \mathrm{kpc}$, we have $M_{v}=-5.0$ at maximum, without allowance for reddening. Eggen (1968) found $B-V=0.30$, at the pre-maximum standstill of the 1967 outburst. The intrinsic colour of a nova near maximum is $B-V$ $\sim 0.0$, so we deduce a visual absorption $\sim 1.0 \mathrm{mag}$ (a maximum value of $A_{v}=1.00$ at the galactic latitude of T Pyx is also obtained from the absorption formula given by Sandage (1972)), giving $M_{v} \sim-6.0$ at maximum. The rate of decline gives $M_{v}=-6.5$ at 
maximum, in reasonable agreement. Thus $M_{v}(d)=1.5$ appears to be reliable.

$V Y$ Aqr. At minimum, $m_{v} \sim 17.0$. The rate of decline during the 1962 outburst (Strohmeier, 1962) was $0^{m} .17$ per day, giving $M_{v}=-8.2$ at maximum and $M_{v}(d) \sim 0.0$.

The evidence cited above strongly suggests that all recurrent novae possess giant secondaries. The mean amplitude of the fast and very fast novae is 12.4 mag and their $\bar{M}_{v}(d)$ is 4.0 (Payne-Gaposchkin, 1957, Table 1.17). The three fast recurrent novae VY Aqr, T CrB and RS Oph have mean amplitude 7.7 mag and $\bar{M}_{v}(d)=-0.3$. From these data we conclude that the true amplitudes of outburst in recurrent novae are the same as those for ordinary novae; the luminosity at minimum comes mostly from the secondary.

\subsection{DWARF NOVAE DURING OUTBURST}

During the dwarf novae outburst $U B V$ photometry generally shows a reddening of the colours (e.g. Krzeminski, 1965). However, this does not necessarily imply a reduction in temperature: at minimum light the flux distribution is determined by the continuum and emission lines and hence the colours are not simply related to temperature. At maximum, spectra show that the flux distribution is almost continuous, with at most only weak absorption and emission lines (Section 7) and small Balmer jump (Zuckermann, 1961). The colours may therefore be more safely interpreted to provide effective temperatures. Table IV lists available observations.

A reddening correction is necessary. All the stars listed lie probably within $300 \mathrm{pc}$ of the Sun so we have adopted $M_{v}=7.5$ throughout, and a reddening of 0.33 mag per kiloparsec. That the interstellar absorption is in general small is shown by the absence of polarization in U Gem (Krzeminski 1965) and SSCyg (Kraft, 1956). The mean colours at maximum are $(B-V)_{0}=-0.09$ and $(U-B)_{0}=-0.84$. This point lies just below the black body line (Figure 1) and corresponds to a colour temperature of $\sim 14500 \mathrm{~K}$.

Because of the small Balmer jump, it is more appropriate to compare the $U B V$ colours with those of supergiants rather than dwarfs. The best fit corresponds to B4Ia $(B-V$ $=-0.12, U-B=-0.82$, Johnson, 1966), which has $T_{\text {eff }}=14000 \mathrm{~K}$ (Humphries et al., 1975; Stalio, 1971). The JKL magnitudes of SSCyg and RX And at maximum (Szkody, 1974), which give values of $V-J, V-K$ and $V-L$ in the range $-0.3 \pm 0.2$, also fit spectral types near B4I.

Direct spectrophotometry of the continuum in SSCyg at maximum light has been made by Hinderer (1949), Zuckerman (1961), Mirzoyan and Kalloglyan (1965) and Walker and Chincarini (1968). These observations, which in general find $T \sim 15000 \mathrm{~K}$, (but in one case Walker and Chincarini found $T>\infty$ ), are of limited value because of the short wavelength ranges used.

Because a dwarf nova during outburst is in a non-equilibrium state, and consequently we may expect a range of temperatures across its surface, it is important to extend our discussion into the far ultraviolet. So far, SSCyg is the only outbursting dwarf nova to be observed by satellite (Holm and Gallagher, 1974). Unfortunately the Celescope experiment did not record any dwarf nova images (Payne-Gaposchkin, private communication).

The OAO-2 observations show that the flux in SSCyg at maximum continues to rise down to $1430 \AA$. Holm and Gallagher compare the flux between $5500 \AA$ and $1430 \AA$ with that from main sequence stars and derive an effective temperature of $16900 \mathrm{~K}$ for no absorption and $19200 \mathrm{~K}$ for $E_{B-V}=0.08$. Comparison of their flux distribution with fluxes for B stars (Humphries et al., 1975) and with 1700-V colours (Code, 1973) 
TABLE IV

Colours of dwarf novae at maximum

\begin{tabular}{llrlrllll}
\hline & $V_{\min }$ & \multicolumn{1}{c}{$B-V$} & $U-B$ & \multicolumn{1}{c}{$b^{0}$} & $E_{B-V}$ & $(B-V)_{0}$ & $(U-B)_{0}$ & Ref. \\
\hline Z Cam & 13.6 & 0.00 & -0.80 & 33 & 0.06 & -0.06 & -0.84 & 1 \\
V436 Cen & 15.4 & 0.05 & -0.78 & 21 & 0.13 & -0.08 & -0.87 & 2 \\
V 442 Cen & 16.5 & 0.02 & -0.69 & 24 & $0.15:$ & $-0.13:$ & $-0.80:$ & 2 \\
WW Cet & 13.6 & -0.05 & -0.78 & -72 & 0.06 & -0.11 & -0.82 & 3 \\
SS Cyg & 12.1 & -0.02 & -0.83 & -7 & 0.02 & -0.04 & -0.84 & 4,5 \\
U Gem & 14.5 & 0.01 & -0.88 & 23 & 0.08 & -0.07 & -0.94 & 6 \\
VW Hyi & 13.4 & -0.02 & -0.75 & -38 & 0.05 & -0.07 & -0.79 & 7,8 \\
VW Hyi & 13.4 & -0.08 & -0.75 & -38 & 0.05 & -0.13 & -0.79 & 9 \\
\hline
\end{tabular}

a Supermaximum

References: (1) Kraft et al. (1969); (2) Bateson (1973); (3) Paczynski (1963); (4) Grant and Abt (1959); (5) Zuckerman (1961); (6) Krzeminski (1976); (7) Marino and Walker (1973); (8) Eggen (1968); (9) Vogt (1974)

confirm that $T_{\text {eff }}$ is near $18000 \mathrm{~K}$. Although the maximum of the flux in SSCyg lies below $1400 \AA$, we do not anticipate that the distribution will depart greatly from that in a B star atmosphere. The spectrum in the visible resembles that of a hot stellar atmosphere so the physical processes are probably similar.

Photometric observations of Z Cha at maximum light (Warner, 1974a), and spectroscopic evidence given in the next section, show that during a dwarf nova outburst it is the disc that becomes very bright. The disc radius at minimum light (Section 13) in SS Cyg is $5.9 \times 10^{10} \mathrm{~cm}$ (Walker and Chincarini, 1968). Then the luminosity at maximum is $2 \pi r_{\mathrm{d}}^{2} \sigma T^{4}=32 L_{\odot}$; this lies just outside the lower luminosity limit estimated by Holm and Gallagher. The consistency of this result with other properties of SS Cyg is tested as follows. Adopting a bolometric correction of -1.5 for $18000 \mathrm{~K}$ (Morton and Adams, 1968) we have, for $L=32 L_{\odot}, M_{v}=2.5$ at maximum and hence $M_{v}=5.8$ at minimum. The orbital period-luminosity relationship (see later in this section) gives $M_{v}=6.7$ for the secondary; Walker and Chincarini found the secondary spectrum to be heavily or totally veiled in the blue and ultraviolet by the overlying continuum; in the yellow the continuum would be weaker and estimate $M_{v} \sim 6.0$ for the system as a whole. Thus the spectroscopic and satellite estimates are in good agreement.

This result is incompatible with the conclusion of Wallerstein (1962) that $M_{v}>8.5$ for SS Cyg. Wallerstein obtained high dispersion coudé plates of SSCyg during an outburst and was unable to detect interstellar lines. His plates, however, showed weak emission lines of hydrogen. Walker and Chincarini (1968) found that the emission lines of $\mathrm{H}$ and $\mathrm{Ca}$ II at minimum in SS Cyg become absorption lines at maximum. The absence of either emission or absorption lines of Ca II in Wallerstein's spectra suggest that they were taken at a time when the lines were filled in and interstellar lines were not detectable.

Strand (1948) measured a parallax of $0^{\prime \prime} .032 \pm 0.010$ for SSCyg, which gives $M_{v}$ $=9.5 \pm 0.8$. Van Maanen (1938), on the other hand, found $\pi=-0.012 \pm 0.012$. Until a modern parallax of high weight is produced (which should be given high priority in view of the extent of recent observations of SS Cyg), there must still remain some uncertainty in the distance of SSCyg. We note that if $\mathbf{M}_{v}=5.8$, then the proper motion gives 
a transverse velocity of $\sim 100 \mathrm{~km} \mathrm{~s}^{-1}$. Although considerably higher than most space velocities of the dwarf novae (Kraft and Luyten, 1965), it is comparable to the $-124 \mathrm{~km}$ $\mathrm{s}^{-1}$ radial velocity of EX Hya (Kraft, 1966).

The uniformity of the unreddened colours in Table IV is indicative that at maximum light the dwarf novae have very similar effective temperatures. Their luminosities will then be proportional to their disc areas; with $a^{3} \propto P^{2}$ we have $L \propto P^{4 / 3}$. SS Cyg will be exceptionally luminous because it has one of the longer orbital periods. The median orbital period for dwarf novae (Table I) is near $4^{\mathrm{h}}$; the median luminosity at maximum is therefore $\sim 32 \times(4 / 6: 6)^{4 / 3}=16 L_{\odot}$, or $M_{v} \simeq 3.2$. The average range in brightness of the dwarf novae in the GCVS is 3.2 visual magnitudes. We must increase this by an amount $-2.5 \log \langle\sin i\rangle=0.8 \mathrm{mag}$ to allow for aspect effects of the disc. Thus we deduce $M_{v}$ $=7.2$ at minimum, which is in excellent accord with the statistical parallax (Kraft and Luyten 1965).

\subsection{THE NOVA-LIKE VARIABLES}

Some of the nova-like variables (those showing strong $\mathrm{He}$ II and $\lambda 4650$ emission) are probably ordinary nova remnants. Many of the stars listed in Table II (iv), however, have broad absorption lines. In Section 7 we show that these lines originate in the disc rather than in the primary. The colours of these objects (Table V) are not greatly affected by the spectrum lines and the Balmer jump is small or non-existent. Their general appearance is like the dwarf novae at maximum. As we find $M_{v} \sim+4.5$ for these objects, we have several objects with distances greater than $300 \mathrm{pc}$ and at high galactic latitude. Table $\mathrm{V}$ contains a crude attempt at estimating the reddening corrections allowing for the effects of distance and latitude.

The unreddened colours lie close to the black body curve in the two-colour diagram and give colour temperatures in the range 12000-19000 K, with the exception of Feige 24 which is apparently of very high temperature.

No satellite observations of the nova-like variables are available. Only for UX UMa do we have an estimate of the disc radius $\left(3 \times 10^{10} \mathrm{~cm}\right.$ : Nather and Robinson, 1974); the colours for this star suggest $T=12000$ which gives $L=1.7 L_{\odot}$. This should be a lower limit because we found higher effective temperatures for the dwarf novae than their $U B V$ colours suggested. In general, the luminosities of the nova-like variables appear on the basis of colours to be similar to that $\left(L=16 L_{\odot}\right)$ found for the dwarf novae at maximum, i.e. $M_{v} \sim+3$.

Confirmation of this luminosity comes from several places. Schild (1969) derived $M_{\nu}=3.7$ for HDE 310376 from arguments based on reddening. Cowley et al. (1975) find $M_{v}<6$ from the space motion of TT Ari. Most important of all, the recent determination of the parallax of $\mathrm{CD}-42^{\circ} 14462$ (Churms 1975) of $\pi=-0.003 \pm 0.006$ gives $M_{v}<5.3$ if we adopt an upper limit on $\pi$ of 0.010 .

\subsection{THE HOT SPOTS}

Smak has drawn attention to the fact that the hot spots in general produce larger humps in the visual light curves of the dwarf novae than in those of ordinary novae and novalike systems. In systems such as U Gem, VW Hyi, Z Cha and Z Cam the hump amplitude is as much as $50 \%$ of the (maximum) light from the system. Even during the inter-hump region the flickering suggests that we still see a substantial contribution from the spot. 
TABLE V

Colours of nova-like variables with absorption lines

\begin{tabular}{llrlrlrc}
\hline & $V$ & \multicolumn{1}{c}{$B-V$} & $U-B$ & \multicolumn{1}{c}{$b^{0}$} & $E_{B-V}$ & $(B-V)_{0}$ & $(U-B)_{0}$ \\
\hline TT Ari & 10.6 & -0.07 & -0.95 & -43 & 0.06 & -0.13 & -0.99 \\
UX UMa & 12.9 & 0.09 & -0.77 & +63 & 0.07 & 0.02 & -0.82 \\
VY Scl & 13.2 & -0.10 & -1.00 & -72 & 0.06 & -0.16 & -1.04 \\
HDE 310376 & 11.2 & 0.00 & -0.92 & -8 & 0.07 & -0.07 & -0.97 \\
CD -42 14462 & 10.3 & 0.02 & -0.75 & -28 & 0.05 & -0.03 & -0.79 \\
LS 55 -8 & 12.8 & 0.07 & -0.79 & -7 & 0.15 & -0.08 & -0.90 \\
Fiege 24 & 12.3 & -0.23 & -1.25 & -50 & 0.08 & -0.31 & -1.31 \\
BD -703007 & 10.6 & 0.00 & -0.75 & +34 & 0.06 & -0.06 & -0.79 \\
\hline
\end{tabular}

Thus the result given above that $M_{v}=7.5$ for dwarf novae should be considered to be the luminosity of their hot spots.

The nova-like variables UX UMa and RW Tri have small and variable hump amplitudes, reaching $0^{m} \cdot 3$ in the former and $0^{m} \cdot 2$ in the latter, but of ten disappearing completely. The low flickering amplitude in these systems also suggests a smaller contribution from the hot spot than is the case for the dwarf novae. If these systems have $M_{v}=+4.5$ then their spots have $M_{v} \sim 6$.

In the nova remnants the situation is less clear. Flickering activity is certainly prominent in the majority, which if originating in the spot suggests a substantial contribution from the spot. DQ Her shows a hump of variable amplitude, occasionally reaching $0^{m} \cdot 15$. RR Pic always has a hump of $0 m^{m}$ amplitude. T Aur possesses no hump. $M_{v}$ at minimum for these systems are 6.8, 5.3 and 4.4 respectively (Payne-Gaposchkin, 1957). In T Aur the disc may therefore dominate and the spot is a small contributor. The evidence, such as it is, suggests $M_{v} \sim 6$ on average for the nova remnants but probably much fainter in DQ Her.

\subsection{LUMINOSITIES OF THE SECONDARIES}

In the case of recurrent novae we have already seen that by adopting absolute magnitudes of normal stars for the secondaries, we get accord with the distances determined from the rate of decline after outburst. In only one case, Nova Per 1901, can we find an independent estimate of the luminosity of the secondary. From the rate of decline, PayneGaposchkin (1957) gives $M_{v}=-8.3$ at maximum; Baade and Humason (1943) found -8.4 from the rate of expansion of the nebular disc. With a range of $13.0 \mathrm{mag}$, these imply $M_{v}=4.7$ at minimum (uncorrected for absorption) and a distance $\sim 460 \mathrm{pc}$. Correction for absorption would give $M_{v} \sim 4.5$ at minimum. Gallagher and Oinas (1974) find from spectra that the K2V-IV secondary of GK Per contributes about one third of the light in the system in the blue. (Note, however, that from our remarks in Section 4, we would expect considerable rotational broadening of the secondary spectrum. As Gallagher and Oinas use line depths to estimate the contribution from the secondary, they may have underestimated its contribution). Hence $M_{B} \sim 4.5+(B-V)+1.2=6.4$, or $M_{v} \sim 5.4$ for a $\mathrm{K} 2$ star. The secondary thus lies about one magnitude above the main sequence, in agreement with the luminosity classification.

In the case of GK Per, the orbital period of $16^{\mathrm{h}} 26^{\mathrm{m}}$ implies an orbital size sufficient to accommodate a Kstar just above the main sequence. For periods less than $\sim 10^{\mathrm{h}}$, however, stars larger than dwarfs would be likely to exceed the size of the Roche lobe. 
Kraft $(1962,1963)$ drew attention to the fact that the luminosities of secondaries deduced from those dwarf novae showing the secondary absorption spectrum (Table I) are incompatible with the statistical parallax of dwarf novae: the secondaries are apparently underluminous for their spectral types. However, this problem was overcome (Kraft and Luyten, 1965) by the recognition that the systems showing secondary spectra are those of longest orbital period (and which therefore have dimensions sufficient to contain the earlier and more luminous dwarfs). There is thus a spread of several magnitudes in the brightness at minimum of dwarf novae and there is no difficulty in reconciling the spectra and the statistical parallaxes. The recent discussion by Gallagher and Oinas (1974) seems to have overlooked this point.

The relationship between orbital period and secondary mass, given in Section 4 , leads immediately to a relationship between orbital period and luminosity of the secondary for those systems containing dwarf secondaries $\left(P \lesssim 10^{\mathrm{h}}\right)$. Equation (6) gives $R_{2} / R_{\odot}=$ $0.11 P(\mathrm{~h})$. A reasonable fit to the $M_{v}$ - radius relationship given by Allen (1973) and the recent radii determined by Bopp et al. (1974) is

$$
M_{v}=-12.5 \log R / R_{\odot}+5.5\left(4 \leqslant M_{v} \leqslant 15\right)
$$

Hence we have the approximate period-luminosity relationship

$$
M_{v}=-12.5 \log P(\mathrm{~h})+17.5
$$

This relationship predicts that at $P=5^{\mathrm{h}}, M_{\nu, 2}=8.8$. Hence in dwarf novae, which have $\bar{M}_{v}=7.5$, we expect that the secondary spectrum should be just visible at $P \sim 5^{\mathrm{h}}$ and increases in prominence for longer orbital periods. This is as observed (Table I).

A small amount of information is contributed by those systems with deep eclipses. Thus we have for U Gem, VZ Scl and Z Cha eclipse depths $\Delta m_{v}$ of $0^{m} \cdot 7,2^{m} .5$ and $2^{m} .0$ respectively, which (adopting $M_{v}=7.5$ ) provide $M_{v, 2}>8.2,10.0$ and 9.5 respectively. All these are consistent with the $P-M_{v, 2}$ relationship, which predicts $M_{v, 2}=9.8,10.8$ and 14.4 respectively for these stars.

\section{Spectrum Line Profiles}

The spectacular nature of the classical and recurrent novae has ensured very complete studies of their spectra during outburst. Most aspects of these studies relate to changes in the structure of the ejected shell and fall beyond the scope of this review. However, there is at least one feature which may be connected with the basic structure of these systems.

The non-spherical structures of nova shells, as deduced from the multiple components of spectral lines and the photographs of novae nebulosities, have been reviewed by Mustel and Boyarchuk (1970). There is clear evidence for axial symmetry, with polar plumes and rings ejected into, or parallel with, the equatorial plane. Several explanations have been suggested; Mustel and Boyarchuk proposed guidance by magnetic fields, Hutchings (1972) interaction of the shell with the binary companion, and Warner (1972b) ejection by the build-up of non-radial oscillations. The latter proposition, while giving a remarkably good fit to the observed plumes and rings, is weakened by the general absence of observed oscillations on the rising branch of the outbursts of dwarf novae (Section 11). This argument presupposes that classical novae and dwarf novae outbursts originate in the same type of 
thermonuclear runaway. The models of the nova outburst computed by Starrfield et al. (1972), however, indicate that the excitation of pulsation modes found in Rose and Smith's (1972) study is not required for shell ejection.

The most appealing explanation of the structure of nova ejecta is that given by Sparks and Starrfield (1973). They suggest that the initially spherically symmetric shell is radically altered by interaction with the disc around the white dwarf. The particular attraction of this theory is that it not only provides the possibility of explaining the structure of novae shells, but also promises an explanation of the different chemical compositions of the various shell ejecta. Thus the equatorially ejected material sweeps with it the circumstellar disc, whereas polar ejecta have a composition more nearly that of the processed white dwarf material. Thus we may expect to see more CNO enhancement (Starrfield et al., 1972) in the polar ejecta than in the equatorial ejecta - where the normal composition of the disc dilutes the enhancements. Differences such as these may explain the two distinct compositions found in V603 Aql by Weaver (1974). If this theory is correct, then the disc mass must be comparable to the mass of the ejected shell in classical novae, i.e. $\sim 10^{-4} M_{\odot}$. By similarity, the discs in all other CV's (in particular the dwarf novae) may be expected to have such masses.

Spectra of dwarf novae during outburst have been moderately well studied (Table VI), but few serious attempts at interpretation have been made. An early study by Adams and Joy (1922) showed that SS Cyg and SS Aur have broad $\mathrm{H}$ and He absorptions at maximum light. Joy (1940) later found that at low dispersion UGem, X Leo and SU UMa have continuous spectra at maximum. The stars RU Peg, RX And and Z Cam were found to have emission lines at maximum.

Some of the conclusions have been modified by more extensive observations with higher resolution. Elvey and Babcock (1943) observed a large number of dwarf novae at various phases in their light curves. A summarizing review has been given by Joy (1960). Many objects show broad $\mathrm{H}$ absorption at or near maximum, variously filled in with emission. In some cases the regions of $\mathrm{Ha}, \beta$ are continuous, whereas $\mathrm{H} \gamma, \delta$ show absorptions, resulting from the emission decrement being larger than the absorption decrement. Other stars show only continua, or Balmer emission. In some stars the absorption lines are visible only before or after maximum - at maximum the lines disappear.

In general the amplitudes of the outbursts are 3 or 4 mag, so we may suspect that the emission lines seen at maximum are of the same strength and origin as those seen at minimum, i.e. arising in the emission disc. Elvey and Babock (1943) show that this is quantitatively correct in the case of SS Cyg, and qualitatively the case in Z Cam. Walker and Chincarini (1968) reach similar conclusions for SS Cyg. These observations show that the part of the disc-the 'chromosphere' - responsible for the emission lines is not greatly affected by the outbursts. As the bright spot contributes significantly to the emission line strengths (especially in SSCyg) this provides evidence that the bright spot is not affected by an outburst. Furthermore, the photometric observations of $U$ Gem made during outburst by Krzeminski (1965), discussed in more detail in Sections 8.3, 9 and 13, show (from the disappearance of eclipses during outburst) that the outburst cannot be due to an increase in luminosity of the bright spot itself. The outburst luminosity therefore most probably comes from the disc or the white dwarf.

To interpret the broad $\mathrm{H}$ and $\mathrm{He}$ absorption lines seen at maximum light we must be able to distinguish between pressure and rotational broadening. If the absorption lines 
TABLE VI

References to spectra of dwarf novae at maximum light

\begin{tabular}{ll}
\hline RX And & $1,2,3$ \\
SS Aur & $1,4,3,5$ \\
SV CMi & 6 \\
Z Cam & $1,2,3,5,7$ \\
SY Cnc & 3 \\
SS Cyg & $1,3,4,5,8,9,15,16$ \\
EM Cyg & 17 \\
AB Dra & 1,3 \\
U Gem & $1,3,5,10$ \\
AH Her & 11 \\
VW Hyi & 18 \\
X Leo & $1,3,5$ \\
CY Lyr & 3,5 \\
CN Ori & 3,5 \\
RU Peg & 1,3 \\
TZ Per & $3,5,12$ \\
UZ Ser & 3,13 \\
SU UMa & $1,3,5,12$ \\
SW UMa & 3,14 \\
\hline
\end{tabular}

1. Joy (1940)

2. Lortet (1968)

3. Joy (1960)

4. Adams and Joy (1922)

5. Elvey and Babock (1943)

6. Bond and Tifft (1974)

7. Lortet-Zuckermann (1967)

8. Hinderer (1949)

9. Greenstein (1960)
10. Krzeminski (1965)

11. Bretz (1965)

12. Herbig (1960a)

13. Herbig (1950)

14. Wellman (1952)

15. Walker and Chincarini (1968)

16. Zuckermann (1961)

17. Robinson (1974)

18. Vogt (1975)

originate in the disc, then rotational broadening will be the dominant mechanism. However, if a significant amount of light at maximum comes from the primary, it is possible that it contributes an absorption spectrum characteristic of a white dwarf. The photometric results do not yet indicate the fraction of light coming from the primary so we must rely on interpretation of the spectra.

The extensive observations of dwarf novae during outbursts by Elvey and Babcock (1943) and the study of SS Cyg by Walker and Chincarini (1968) show that absorption line half-widths up to $50 \AA$ occur. Such widths are found in normal white dwarfs and are caused by pressure broadening. However, rotational broadening in the disc can also produce broad lines and would account for their shallowness. The Keplerian velocity at the surface of a $0.8 M_{\odot}$ white dwarf is $\sim 4000 \mathrm{~km} \mathrm{~s}^{-1}$, which gives a line width $\sim 50 \AA$ at $\mathrm{H} \gamma$.

Fortunately, the existing observations are sufficient to establish that rotational effects cause the broad lines. Firstly, Elvey and Babcock found that the widths of the absorption lines at maximum in $\mathrm{ZCam}$ are the same as the widths of the emission lines seen at minimum in that star. It would be an unlikely coincidence if the rotationally broadened disc emission lines had exactly the same width as pressure-broadened lines in the outbursting primary. Secondly, Walker and Chincarini's spectra show that at minimum SS Cyg has single $\mathrm{H}$ emission lines with wings extending out to $3000 \mathrm{~km} \mathrm{~s}^{-1}$. The inclination of the orbit of SS Cyg is $\sim 43^{\circ}$ (Table III), so we are seeing $\mathrm{H}$ emission from regions 
with velocities $\sim 4400 \mathrm{~km} \mathrm{~s}^{-1}$ near the surface of the primary. In addition, there is weak, double Ca II emission with components separated by $\sim 450 \mathrm{~km} \mathrm{~s}^{-1}$. We interpret this to imply that the $\mathrm{Ca}$ II is concentrated in the outer parts of the disc, whereas $\mathrm{H}$ emission is present over the entire disc. The inner parts of the disc are probably hot enough to ionize the Ca II. A similar circumstance may obtain in SS Aur. Kraft (1962) says that the continuum in SS Aur is irregular and may correspond to the spectrum of a late-type star. However, on the reproduction of the spectrum given in his paper, the writer feels that the continuum uneveness is due to emission lines of Fe II (the same ones that appear in $\mathrm{U}$ Gem), and these are double whereas the $\mathrm{H}$ lines are single.

At maximum light a mirror image is seen; broad, single $\mathrm{H}$ absorption and doubled $\mathrm{Ca}$ II absorption (now with line components separated by $\sim 800 \mathrm{~km} \mathrm{~s}^{-1}$ ). The line doubling in $\mathrm{Ca}$ II has not previously been satisfactorily explained, but it certainly cannot arise from pressure-broadening. We suggest that it is a simple consequence of rotational broadening where again the $\mathrm{Ca}$ II is concentrated in the outer parts of the disc (but now the distribution is different to that at minimum light and extends further in towards the centre of the disc). The $\mathrm{H}$ absorption lines are single because again they originate throughout the disc.

We conclude that absorption line profiles during outbursts of dwarf novae potentially can tell us something about the distribution of physical conditions across the disc. For instance, there is the study of UZ Ser by Herbig (1950), who found $\mathrm{H}$ and $\mathrm{He}$ absorption line widths of $\sim 1060 \mathrm{~km} \mathrm{~s}^{-1}$ just after maximum, increasing to $\sim 1900 \mathrm{~km} \mathrm{~s}^{-1}$ a few days later (with only a few tenths of a magnitude drop in brightness). This probably indicates that the disc is contracting after maximum, but alternatively it could be that shortly after maximum most light comes from outer regions of the disc, but later the inner regions dominate.

WZ Sge also shows broad absorption lines (Greenstein, 1957; Krzeminski and Smak, 1971 ), in the centres of which are double emission lines. The parallax and proper motion (Van Maanen, 1926) both suggest a white dwarf luminosity for WZ Sge, so it is conventional to interpret the absorption lines as being pressure broadened. An argument in favour of this is that the wings of the lines can be traced out to $100 \AA$ from the line centre, i.e. to $\sim 7500 \mathrm{~km} \mathrm{~s}^{-1}$. This may appear greater than the Keplerian velocity at the surface of a white dwarf. However, our later discussion of $\mathrm{CD}-42^{\circ} 14462$, which is certainly not a simple white dwarf, shows that such broad lines are possible from a disc. Interpreted as a white dwarf, WZ Sge has $M_{v} \sim+11$ (Krzeminski and Smak, 1971) and consequently is the faintest of the CV's. Its outburst amplitude of 7 mag thus results in $M_{v} \sim+4$ at maximum, which is similar to that of the dwarf novae. McLaughlin (1953, 1960 ) points out that at the end of outburst (unfortunately no spectra have been obtained during the maximal phases of outburst) no nebular or high excitation lines appear. This signifies small or absent mass ejection, behaviour which again is consistent with the dwarf novae. McLaughlin's study also shows that while WZ Sge was still one magnitude above minimum light, the broad absorption and double emission lines were visible - as at minimum (he observes absorption at $-2440 \mathrm{~km} \mathrm{~s}^{-1}$ in $\mathrm{H} \gamma$ and $-3700 \mathrm{~km} \mathrm{~s}^{-1}$ at $\mathrm{H} \beta$; these are the 'centres' of the shortward absorption, corresponding to the position of deepest absorption. The wings probably extend to $7500 \mathrm{~km} \mathrm{~s}^{-1}$ as at minimum). In WZ Sge, therefore, as in the dwarf novae, it looks as if it is the disc that brightens during outburst. A reinterpretation of WZ Sge in terms of a bright disc rather than a white dwarf is strongly 
suggested. A redetermination of the parallax and proper motion is urgently required.

DI Lac is the only classical nova showing broad absorption lines at minimum light (Greenstein, 1960; Kraft, 1963, 1964a). Kraft finds no radial velocity variations larger than $25 \mathrm{~km} \mathrm{~s}^{-1}$, which would imply either an almost pole-on system or a large mass ratio (as in WZ Sge). The pole-on situation is the most probable because the emission cores in the centres of the broad absorption are the narrowest observed in any nova remnant. The total width of the absorption lines is only a quarter of that seen in WZ Sge; this, and Kraft's (1963) statement “. . . their profiles are decidedly 'dish-shaped' - more like a rapidly rotating star than a white dwarf' is suggestive that in DI Lac we see the absorption spectrum of the disc. If interpreted as a white dwarf spectrum the absolute magnitude would be fainter than +10 . However, the spectral development during the outburst of 1910 was quite normal for a fast nova, as was the outburst amplitude; these provide an estimate of $M_{v} \sim+2.5$ at minimum light (Payne-Gaposchkin, 1957). This is the brightest of the nova remnants listed by Payne-Gaposchkin, with the exception of HR Lyr which has $M_{v} \sim+2.1$. HR Lyr also has an almost continuous spectrum, with only very weak and narrow emission lines. DI Lac and HR Lyr had outburst amplitudes of 9.8 and 8.8 mag respectively, which puts them close to the range $\left(9^{m} \cdot 1\right)$ of WZ Sge and which are the smallest amplitudes known for classical novae.

These considerations lead us to suggest that in DI Lac and HR Lyr the disc is considerably more luminous than normal and thus the minimum magnitude is abnormally bright. Consequently, the true amplitude of outburst is greater than the apparent range.

Among the nova-like variables, the following (c.f. Table II (iv)) show broad absorption lines with emission cores: TT Ari, UX UMa, BD $-7^{0} 3007, \mathrm{CD}-42^{0} 14462$, HDE 310376 , LS $55^{\circ}-8$, Feige 24 and VYScl. Several of these show both $\mathrm{H}$ and $\mathrm{He}$ absorption, in contradistinction to the dichotomy that exists between DA and DB white dwarfs. This is a strong clue that we are not dealing with spectra formed in the atmospheres of white dwarfs; the more natural interpretation is that the spectra arise in the atmospheres of the accretion discs which, having the same composition as the secondary, have spectra more like those of normal OB stars.

It has been suggested (Warner and Van Citters, 1974) that these stars could be Z Cam stars permanently stuck in the standstill condition. Such a hypothesis accounts for the similarity between the spectra of these objects and the spectra of dwarf novae during outburst, and the flickering amplitude, which is generally much lower than that in nova remnants and dwarf novae. With $M_{v} \sim+7.5$ for the dwarf novae, the absorption line novalike objects would have $M_{v} \sim+4.5$ if the above hypothesis is correct. This agrees with the evidence on absolute magnitudes given in Section 6.

The wings of $\mathrm{H} \gamma$ in $\mathrm{CD}-42^{\circ} 14462$ (Wegner, 1972) extend to $110 \AA$ from the line centre. As in the case of WZ Sge, this leads to a rotational velocity well in excess of the Keplerian velocity around a white dwarf - especially when we consider that $C D-42^{\circ}$ 14462 does not eclipse and therefore is of lower inclination. The fact that we can see any hydrogen absorption from material with such a high velocity implies that the inner regions of the disc must contribute a large proportion of the light in the visible. We suggest that the gas near the surface of the white dwarf is both rotating rapidly and at high pressure, so that absorption lines from this region show both doppler and pressure broadening.

We note that in (a) the dwarf novae during outburst, (b) the nova-like variables discussed above and (c) the unusual nova remnants DI Lac and HR Lyr, we find very weak 
emission lines. We have already pointed out that the emission line strength appears not to vary during the outburst of a dwarf nova; coupled with (b) and (c) and our explanation of these objects in terms of bright discs, we reach the conclusion that emission line strengths in CV's are independent of disc luminosities. Thus the excitation mechanism for the lines cannot be the disc radiation. On the other hand, we have seen that the emission lines and the continua in nova remnants have ten times greater intrinsic intensity than in dwarf novae. All of these properties can be understood if the excitation results from the mass transfer process, which we conclude does not vary during outbursts, but is about ten times greater in novae than dwarf novae. Additional evidence for the independence of emission lines and disc properties comes from the observed variations in emission line profile around the orbital cycle of CV's at normal light. In general, CV's observed at fairly large inclination $\left(i \geq 50^{\circ}\right)$ show doubled emission lines from the disc. Published spectra of U Gem (Kraft, 1962), DQ Her (Greenstein and Kraft, 1959) and RW Tri (Walker, 1971) show that the intensity ratio of the two components varies systematically around the orbit, with the shortward component being most intense at phases between 0.6 and 0.8. In other objects (e.g. VV Pup: Warner and Nather, 1972a) in which the lines are single, the emission line strength also reaches a maximum near phase 0.7 . These spectral variations indicate that the emission lines are strongest in the vicinity of the hot spot. The existence of such strong variations in line intensity around the disc again suggest that the disc itself is not the source of excitation.

During the outbursts of some dwarf novae, the doppler-broadened absorption lines are wider than the corresponding emission lines. This is probably a consequence of the emission lines arising from the region of the hot spot and from the outer parts of the disc, the absorption coming from the whole disc.

Line profiles of absorption lines in the secondaries should be dominated by rotational broadening equal to an equatorial velocity of $128 \mathrm{~km} \mathrm{~s}^{-1}$ (Section 4). Evidence for such broadening comes from several sources. In AE Aqr (Joy, 1954) line widths are $2.0 \AA$ giving $v \sin i \sim 73 \mathrm{~km} \mathrm{~s}^{-1}$; from Table III we have sin $i \simeq 0.73$, giving $v \simeq 100 \mathrm{~km} \mathrm{~s}^{-1}$ in reasonable agreement with prediction. On tracings of the spectra of SS Cyg (Walker and Chincarini, 1968), kindly made available by Dr M. F. Walker, we find $v \sin i \simeq 100 \mathrm{~km}$ $\mathrm{s}^{-1}$, after correction for instrumental resolution, so that $\nu \sim 146 \mathrm{~km} \mathrm{~s}^{-1}$. Young and Nelson (1972) state that in $\mathrm{BD}+16^{\circ} 516$ the lines are considerably broadened by rotation.

\section{Conditions in the Disc}

The previous section has shown that we have a number of observational clues pointing the way to a description of the physical conditions in the disc. We will consider separately (8.1) the low density line emitting region - the chromosphere, (8.2) the continuum emission region - the hot spot, and (8.3) the main body of the disc itself.

\subsection{THE CHROMOSPHERE}

The electron density in the chromosphere may be found by using the total emission in $\mathrm{H} \beta$. For a chromosphere thickness $d_{\mathrm{c}}$ and area $A_{\mathrm{c}}$ we have for optically thin emission

$$
I(\mathrm{H} \beta)=\alpha_{4} f N_{\mathrm{e}}^{2} h v A_{\mathrm{c}} d_{\mathrm{c}}
$$

where $\alpha_{4}$ is the recombination rate onto the fourth level $\left(\alpha_{4} \sim 3 \times 10^{-14} \mathrm{~cm}^{3} \mathrm{~s}^{-1}\right.$ at 
$20000 \mathrm{~K})$ and $f$ is the fraction of transitions from the fourth level that go into $\mathrm{H} \beta$.

No absolute spectrophotometry is available for CV's, but we may estimate $I(\mathrm{H} \beta)$ as follows. A typical equivalent width for the $\mathrm{H} \beta$ emission is $\sim 30 \AA$. Adopting $M_{\nu}=7.5$ for dwarf novae we have $I(5000 \AA) \sim 1.1 \times 10^{28} \mathrm{erg} \mathrm{s}^{-1} \AA^{-1}$ and hence $I(\mathrm{H} \beta) \sim 3.3 \times 10^{29}$ ergs $\mathrm{s}^{-1}$.

Then

$$
N_{\mathrm{e}} \sim 5 \times 10^{11}\left(\frac{10^{10}}{d_{\mathrm{c}}}\right)^{\frac{1}{2}}\left(\frac{10^{21}}{A_{\mathrm{c}}}\right)^{\frac{1}{2}} \mathrm{~cm}^{-3}
$$

The area of the disc contributing to the emission lines is probably somewhat less than $\pi r_{d}^{2}$, so $N_{\mathrm{e}} \sim 1 \times 10^{12} \mathrm{~cm}^{-3}$.

The Balmer Series is seen in emission with upper principal quantum number $\mathrm{n}$ reaching up to $\sim 16$. However, an application of the Inglis-Teller formula (which would give $N_{\mathrm{e}} \sim 10^{14} \mathrm{~cm}^{-3}$ ) is inappropriate because of the extreme rotational broadening of the emission lines. Variations of $n$ (between spectra of the same object taken at different times) occur and presumably arise from differing distributions of emission over the disc.

The rotation period of the outer part of the disc $2 \pi_{d}^{3 / 2}\left(G M_{1}\right)^{\frac{1}{2}}$ is $\sim 1500$ s and the line emission falls significantly in intensity in a quarter of a period. The apparent cooling time is therefore $\sim 400 \mathrm{~s}$. If electron collisions are the dominant source of ionization, then the cooling time may be estimated by noting that for $10^{4}<T<10^{5} \mathrm{~K}$ the power radiated by an optically thin plasma is $P_{r} \sim 1 \times 10^{-22} N_{\mathrm{e}}^{2} \mathrm{ergs} \mathrm{cm}^{-3} \mathrm{~s}^{-1}$ (Cox and Tucker, 1969). The radiative cooling time $3 N_{\mathrm{e}} k T / P_{r}$ is then $\sim 0.3 \mathrm{~s}$. The ionization of the chromosphere is therefore maintained by a radiation field which, from the concentration of the emission around the region of the hot spot, must arise from the hot spot itself.

\subsection{THE HOT SPOT}

The observations discussed in Sections 2 and 3 show that whereas the Balmer emission lines are present over much of the disc, the continuum (and occasionally some of the higher excitation lines-e.g. He I in U Gem) arises principally in the vicinity of the hot spot. The Balmer jump is often seen in emission; in SSCyg (Walker and Chincarini, 1968, Figure 4) the continuum at $3650 \AA$ has twice the intensity at $4200 \AA$, but this should be increased to $\sim 2.5$ times to remove the contribution from the G-type secondary. Such a Balmer jump indicates emission from a $\sim 30000 \mathrm{~K}$ plasma (Kunkel, 1970), in tolerable agreement with the temperature found from OAO-2.

We already found that $I(5000 \AA) \sim 1.1 \times 10^{28} \mathrm{erg} \mathrm{s}^{-1} \AA^{-1}$ for dwarf novae; the emission for an optically thin hydrogen plasma at $3 \times 10^{4} \mathrm{~K}$ is $\sim 3 \times 10^{-29} N_{\mathrm{e}}^{2} \mathrm{erg} \mathrm{cm}^{-3} \mathrm{~s}^{-1} \AA^{-1}$ near $5000 \AA$ (Kunkel, 1970). We therefore find $N_{\mathrm{e}} \sim 6 \times 10^{14}\left(l / 10^{9}\right)^{-3 / 2}$ for dwarf novae and a factor of three greater for nova remnants, where $l^{3}$ is the volume of the hot spot.

The free-free opacity (Allen, 1973) is $K_{\mathrm{ff}} \sim 1.37 \times 10^{-47} \lambda^{3} N_{\mathrm{e}}^{2} T^{\frac{1}{2}}$, giving $K_{\mathrm{ff}} \sim 1.0$ $\times 10^{-8} \mathrm{~cm}^{-1}$. The hot spot region in the visible is therefore optically thick and the $N_{\mathrm{e}}$ derived above must be considered a lower limit. We therefore have $N_{\mathrm{e}} \geqslant 10^{15} \mathrm{~cm}^{-3}$ in the outer parts of the hot spot. For $l \sim 10^{9} \mathrm{~cm}$ the hot spot would be optically thick at least down to $1500 \AA$, accounting for the fact that we see roughly the same $3 \times 10^{4} \mathrm{~K}$ surface temperature throughout the spectral region so far studied. Below $1000 \AA$ it 
may be possible to see the small hot core of the hot spot, but this will depend critically on how much material is in the vicinity of the spot or the system as a whole, and may depend on orientation of the system as well. It should be noted that the decomposition of the flux distribution by Szkody (1974) into contributions from regions of temperatures $4 \times 10^{5} \mathrm{~K}$ and $3 \times 10^{4} \mathrm{~K}$ is incorrect because she has arbitrarily assumed equal volumes for the two regions.

The luminosity of the hot spot is maintained by the conversion of kinetic energy in the colliding gas stream. If the particles leaving the inner Lagrangian point approximately conserve their angular momentum about the primary, then the relative velocity of impact on the disc is $v\left(r_{d}\right) \cos \gamma$, where $\gamma$ is the angle to the radius vector made by the stream as it hits the disc. The temperature of the shock wave generated in the bright spot will then be (Saslaw, 1968)

$$
T_{s} \sim \frac{3 m_{\mathrm{H}}}{32 k} \frac{G M_{1}}{r_{d}} \cos ^{2} \gamma \mathrm{K}
$$

Using Equation (9), the values of $\gamma$ from Flannery (1975), and values of $q$ given in Table III we find that the common binaries with $P \sim 4^{\text {h }}$ have $T_{s} \sim 2 \times 10^{6} \mathrm{~K}$ and the shortest period binaries have $T_{s} \sim 4 \times 10^{6} \mathrm{~K}$. The peak of radiation emitted from such shock waves will therefore lie in the soft $\mathrm{X}$-ray region near $0.3 \mathrm{keV}$. With the hot spot optically thick longward of $1000 \AA$ thermalisation takes place, resulting in the $\sim 30000 \mathrm{~K}$ temperature actually observed. For such an optically thick hot spot, the cooling time is $\sim l^{2} \sigma T^{4} / L$ $\sim 1 \mathrm{~s}$ for $L=1 L_{\odot}$ and $T=3 \times 10^{4} \mathrm{~K}$. Thus the observed flickering at optical wavelengths should not have much power at timescales shorter than $1 \mathrm{~s}$.

For wavelengths shorter than $1000 \AA$ the spot may be optically thin and X-rays may emerge without difficulty. Most of the cooling of the spot will then take place from radiation behind the shock front. The X-ray luminosity is given by (Blumenthal and Tucker, 1974)

$$
L \sim 2.4 \times 10^{-27} T^{\frac{1}{2}} N_{\mathrm{e}}^{2} \mathrm{erg} \mathrm{s}^{-1} \mathrm{~cm}^{-3},
$$

and the cooling time is therefore $\tau_{\mathrm{c}} \sim 10^{11} T^{\frac{1}{2}} N_{\mathrm{e}}^{-1} \mathrm{~s}$. The thickness of the high temperature region will be $l_{s} \sim \tau_{\mathrm{c}} v$ where $v\left(\sim 5 \times 10^{7} \mathrm{~cm} \mathrm{~s}^{-1}\right)$ is the velocity of gas through the shock. For $N_{\mathrm{e}} \sim 10^{15} \mathrm{~cm}^{-3}$ we therefore have $l_{s} \sim 10^{7} \mathrm{~cm}$. This very narrow high temperature region has been omitted from the hydrodynamical calculations of gas flow in close binary systems (Prendergast and Taam, 1974; Flannery, 1974; Sorenson et al., 1975) which use rather wide mesh points and time steps.

From the above, the X-ray luminosity of the hot spot is

$$
L \sim 2.4 \times 10^{-27} T^{\frac{1}{2}} N_{e}^{2} l^{2} l_{s}
$$

If the soft X-ray emission of $3.1 \times 10^{-12} \mathrm{erg} \mathrm{cm}^{-2} \mathrm{~s}^{-1}$ found in SS Cyg in its quiescent state (Heise et al., 1975) arises from the hot spot, then (with a distance to SSCyg of $180 \mathrm{pc}$ : Section 6) we have $N_{\mathrm{e}} \sim 2 \times 10^{15} \mathrm{~cm}^{-3}$ for $l=3 \times 10^{9} \mathrm{~cm}$.

Because of the short $\left(\sim 10^{-2} \mathrm{~s}\right)$ cooling time of the shocked gas, luminosity variations in the X-ray region may be more rapid than those already observed in the visible.

Henry et al. (1975) have given upper limits to the flux from RX And and UGem at energies of 0.04 and $0.13 \mathrm{keV}$. They choose to interpret their results in terms of models having temperatures of $2 \times 10^{5} \mathrm{~K}$. If we adopt $2 \times 10^{6} \mathrm{~K}$, then their results imply total 
$\mathrm{X}$-ray luminosities less than $1 L_{\odot}$. This result is unfortunately quite sensitive to the adopted temperature and the assumed attenuation by interstellar and circumstellar hydrogen. A limit a factor of about 4 lower is set by the possible observation of $0.25 \mathrm{keV}$ X-rays from U Gem (Novick and Woltjer, 1975).

Three tentative identifications of cataclysmic variables have been proposed for objects in the Uhuru catalogue of X-ray sources. These are IM Nor (Elliot and Liller, 1972), which is a nova remnant and in which the X-rays may in some way be associated with the ejected gas, EX Hya (Warner, 1972c, 1973c) which is a dwarf nova, and He 2-177 (Carlson and Henize, 1974) which is a probable slow-nova. In the case of EX Hya, the orbital period is $98 \mathrm{~min}$ and we expect the shock temperature to be $\sim 4 \times 10^{6} \mathrm{~K}$. In the Uhuru catalogue (Giacconi et al., 1974) 3U 1252-28 has a flux of 4.5 units, corresponding to $7.7 \times 10^{-11} \mathrm{erg} \mathrm{s}^{-1}$. We adopt $M_{v}=7.5$, which gives a distance of $175 \mathrm{pc}$ for EX Hya; at this distance, the proper motion (Luyten and Hughes, 1965) gives a transverse velocity of $96 \mathrm{~km} \mathrm{~s}^{-1}$, which is similar to the radial velocity of $-124 \mathrm{~km} \mathrm{~s}^{-1}$ measured by Kraft and Luyten, 1965).

Thus the total X-ray flux in the Uhuru passband (2.0-6.0 keV) emitted by EX Hya is $2.8 \times 10^{32} \mathrm{erg} \mathrm{s}^{-1}$. The total X-ray flux for a $4 \times 10^{6} \mathrm{~K}$ black body would be $2 \times 10^{33}$ $\mathrm{erg} \mathrm{s}^{-1}=0.5 L_{\odot}$. This luminosity, however, is about 100 times that found from the soft X-rays from SSCyg.

\subsection{THE DISC}

For the majority of CV's at minimum light we have little direct information about the disc - the spectra in the visible region are dominated by the emission lines from the chromosphere and the continuum from the hot spot. The broad absorption lines that are present in some nova-like variables, and in particular in dwarf novae during outburst show that the discs are optically thick. This provides a crude lower limit for the mass $M_{d}$ and density $\rho_{d}$ of the disc: if the thickness of the disc is $\mathrm{z}$ we have $N_{\mathrm{e}} \sigma_{\mathrm{e}} z>10$, hence $M_{d} \sim \pi r_{d}^{2} N_{\mathrm{e}} z m_{\mathrm{H}} \gtrsim 10^{-11} M_{\odot}$ and $\rho_{d} \gtrsim 10^{-8} \mathrm{gm} \mathrm{cm}^{-3}$.

Smak $(1971 b, 1972)$ has proposed a mechanism to account for the period variations discussed in Section 6. His suggestion that angular momentum stored in the disc by accretion at one time scale, is transferred back to the orbit on another time scale has received support from Biermann and Hall (1973) in their explanation of period changes in Algol systems. In the CV's Smak shows that $M_{d}$ must lie in the range $10^{-6}$ to $10^{-4} M_{\odot}$ in order for the disc to store sufficient angular momentum. From a simple model for the disc cooling after an outburst, Warner $(1974 \mathrm{~b})$ found $M_{d} \sim 3 \times 10^{-5} M_{\odot}$.

In Section 7 we drew attention to the fact that the nonspherical outbursts of novae, as interpreted by Sparks and Starrfield (1973), imply disc masses in the novae of $\sim 10^{-4}$ $M_{\odot}$.

Smak's (1971a) discussion of U Gem reveals an important property of the disc. Although the brightness of the system recovers about 10 days after an outburst, the disc, which initially expands by some $30 \%$ during the initial stage of the outburst, is still contracting some 100 days later when the next outburst starts. The effect of an outburst is therefore 'remembered' by the system for at least 100 days. If this memory resides in the disc itself, then the turn-over time of the disc must be also at least $100 \mathrm{~d}$. On the other hand, the disc may be responding to additional heating coming from the primary, which may take greater than $100 \mathrm{~d}$ to cool after an outburst. 
Krzeminski's observations and Smak's (1971a) interpretation indicate that during the initial rise in U Gem no additional light source is eclipsed, whereas during the final rise the eclipse shifts in phase so that a source centred on the primary (but of larger radius than a white dwarf) is being obscured. Thus over the $\sim 1 \mathrm{~d}$ rise to maximum in dwarf novae the brightening of the disc starts in the centre and spreads outwards. The lifetime of the disc would thus appear to exceed $1 \mathrm{~d}$ and may exceed $100 \mathrm{~d}$. With mass transfer rates into the disc of $\sim 10^{18} \mathrm{gm} \mathrm{s}^{-1}$ (Section 10) we deduce lower limits to the disc mass of $10^{-9}$ or $10^{-7} M_{\odot}$.

The absence of significant polarization in SS Cyg and Z Cam (Landstreet and Angel, 1971 ) suggests the absence of any large magnetic fields in the disc.

\section{The Bright Spot during Outbursts}

Photometrically, the bright spot makes its presence felt in two ways - the periodic hump and the rapid flickering. We obviously want to discover what effects an outburst may have on the bright spot; any changes we see may relate to changes in the structure of the disc or in the rate of mass transfer or other properties of the inter-star stream.

The first relevant observations were made by Grant (1955). He observed SSCyg at minimum $\left(m_{v} \sim 11.3\right)$, on the rise $\left(m_{v} \sim 9.5\right)$ and at maximum $\left(m_{v} \sim 8.9\right)$. From his chart recorder tracings he discovered that the amplitude of the rapid flickering is constant in intensity units during the 3 mag outburst. Grant was very perplexed by this result; he realised that the flickering could not arise in the outbursting star and was forced into suggesting that the flickering originates in the emission lines (which were known not to change in average intensity during an outburst from the work of Elvey and Babcock (1943)). Later Zuckermann (1961), using more extensive observations, also found constant amplitude for the flickering during outbursts of SS Cyg.

As we now know that the bright spot is the source of flickering activity, we see that these early observations give a first indication that during an outburst the spot is not greatly affected.

More quantitative work has recently been carried out by Robinson. In AH Her (Robinson, 1973b) the flickering at maximum light is about twice that at minimum. In RX And (Robinson, 1973c) the flickering amplitude is $40 \%$ greater at maximum light than at minimum. Extensive photometry of Z Cam (Robinson, 1973a) showed that an increase in flickering amplitude by a factor of two occurs as the star brightens the first $1.5 \mathrm{mag}$, the flickering amplitude then decreases until at maximum it is little greater than the value at minimum light.

Other evidence for changes in the bright spot comes from observations of the periodic hump. Krzeminski (1965) found that, during outbursts of U Gem, the intensity of light contributed by the hump stays constant to within a factor of two. Smak (1971a; see his Table 4) found from an analysis of Krzeminski's observations, plus those of Mumford (1964) and Paczynski (1965b), that the hump stays constant in amplitude to within $20 \%$.

Vogt (1974) found the hump in the light curve of VW Hyi during an ordinary outburst to behave in just the same way as that for U Gem.

All of the evidence cited so far shows that only relatively small changes in the bright spot occur during ordinary outbursts of dwarf novae. Robinson (1973a, 1973c) has in fact explained the minor changes that are observed in terms of a small expansion of the 
disc during outburst (as deduced in the case of U Gem by Smak (1971a)) and a small increase in mass transfer caused by heating of the main sequence secondary by the bright primary and disc. Therefore, in agreement with our deductions from the spectroscopic evidence presented in Section 7 we conclude that the rate of mass transfer is not greatly changed during an outburst.

During supermaxima (cf. Section 11) a complication arises. Again there is evidence that the bright spot does not change in intensity. Observations of VW Hyi during its rise (Warner, 1975a) show that as this star rises in brightness by a factor of ten, the hump amplitude remains constant. Vogt (1974) confirms that the normal hump disappears during supermaximum, and reappears with normal amplitude well down the recovery part of the outburst light curve. However, the independent observations of Warner and Vogt (and their discussion of observations by Marino and Walker (1974)) show that during a supermaximum periodically recurring humps appear. These super-humps, near outburst maximum, have an amplitude of $25 \%$ and thus are some 40 times the height of the normal hump. Their amplitude decays more rapidly after maximum than the overall brightness of the star.

The most surprising property of the superhumps is that they have a different period from the normal humps. The orbital period of VW Hyi, deduced from the normal humps, is $P=0.0742711$. By combining the observations by Warner and himself, Vogt found that the super-hump recurs according to the elements $P_{0}=0.07751$ and $\mathrm{d} P / \mathrm{d} t=-5.0$ $\times 10^{-3}$. The 'mean' period during a supermaximum is $\sim 0.0768$, i.e. some $3 \%$ longer than the orbital period. The same super-hump elements were found in two different supermaxima and a third supermaximum, extensively observed by Vogt (1975) has again shown the same behaviour. It may not be coincidence that the time $\frac{1}{2}\left(P-P_{0}\right)(\mathrm{d} P / \mathrm{d} t)^{-1}$ required for the super-hump period to return to the orbital period is $\sim 32^{d}$, i.e. very close to the mean recurrence period $\left(28^{\mathrm{d}}\right)$ of VW Hyi.

A superhump has also been seen during a maximum of V436 Cen (Warner, 1975b) and may be present during supermaxima of Z Cha (Warner, 1974a, 1975a).

\section{Mass Transfer and Loss}

The rate of mass transfer from the secondary to the primary is probably the single most important parameter determining the properties of CV's. The brightness of the hot spot, the outburst frequency, the mass of the disc and the lifetimes are all directly related to mass transference.

The most direct way of establishing the rate of mass loss from the secondary $-\dot{M}_{2}$ is to consider the hot spot. Its luminosity derives from conversion of kinetic energy of the inter-star stream into heat. This conversion may not be completely efficient - some of the material may splash away from the hot spot and be lost from the system; some of the energy may go into turbulence. However, most of the energy in the stream will ultimately appear as radiation, so for those systems which are dominated by the radiation from the hot spot we may write

$$
\begin{aligned}
-\dot{M}_{2} & \sim \frac{4 L}{V_{d}^{2}} \\
& \sim \frac{2 L r_{d}}{G M_{1}}
\end{aligned}
$$


where $V_{d}$ is the relative velocity of impact of the stream onto the disc. Adopting the luminosities given in Section VI we have $-M_{2} \sim 1 \times 10^{18} \mathrm{gm} \mathrm{s}^{-1}$ for dwarf novae and $\sim 1 \times 10^{19} \mathrm{gm} \mathrm{s}^{-1}$ for classical novae. Krzeminski and Smak (1971) found $-M_{2} \sim 3$ $\times 10^{16} \mathrm{gm} \mathrm{s}^{-1}$ from a consideration of the bright spot in WZ Sge. Our suggestion (Section 7) that WZ Sge be re-interpreted as a star with a bright accretion disc implies an increase in absolute magnitude by $\sim 5 \mathrm{mag}$ and a corresponding increase in $-\dot{M}_{2}$ by $\sim 100$.

In principle, the rate of mass transfer should also be deduceable from orbital period variations. In CV's it is difficult to define accurately the time of hump maximum because of flickering, and some phase jitter occurs in the position of mid-eclipse because of motions of the bright spot. However, the short periods result in a large number of cycles per year and consequently period changes $\mathrm{d} P / \mathrm{d} t \sim 10^{-11}$ are detectable.

UX UMa and RW Tri clearly show period changes that are alternating and roughly sinusoidal with periods of tens of years (Mandel, 1965; Nather and Robinson, 1974) AM CVn has cyclical period variations on a timescale 736 days (Krzeminski, 1972). Recent discussions (Smak, 1972; Pringle, 1975) conclude that, as well as the above objects, only T Aur and EM Cyg have definitely established period variations. To these we may add SSCyg in which radial velocity measurements show a change of period (Walker and Chincarini, 1968; Walker and Reagan, 1971) and AE Aqr (Chincarini, private communication). In all of these cases $\tau_{P}=P(\mathrm{~d} P / \mathrm{d} t)^{-1}$ is $\sim 10^{6} \mathrm{yr}$.

There is considerable uncertainty in the interpretation of these changes. Smak's (1972) model of angular momentum interchange between the disc and the secondary shows that instantaneous values of $\tau_{p}$ may give a misleading impression of $\dot{M}_{2}$. In the simplest models we have $\dot{M}_{2}=-\left(\dot{M}_{1}+\dot{M}_{2}\right) / 2 \tau_{P}$ for spherically symmetric mass loss (Nather and Warner, 1969) and $\dot{M}_{2}=-M_{2} / 3 \tau_{P}(1-q)$ for conservation of mass and angular momentum (Pringle, 1975). According to the latter formula we should find that $\mathrm{d} P / \mathrm{d} t$ is negative for $q>1$. AE Aqr and SS Cyg have $\mathrm{d} P / \mathrm{d} t>0$, T Aur and EM Cyg have $\mathrm{d} P / \mathrm{d} t<0$. These are in reasonable accord with the values of $q$ suggested in Table III. With $-\dot{M}_{2} \sim \dot{M}_{2} / 2 \tau_{P}$ we have $-\dot{M}_{2} \sim 2 \times 10^{19} \mathrm{gm} \mathrm{s}^{-1}$, but with great uncertainty as to the value of this estimate.

The 71-s oscillations in the light curve of DQ Her (Section 12) show a rate of change $\tau_{p}=p(\mathrm{~d} P / \mathrm{d} t)^{-1}=-2.64 \times 10^{6} \mathrm{yr}$ (Herbst, Hesser and Ostriker, 1974). The interpretation of the 71-s oscillations is controversial, but we note that if they are non-radial oscillations (Warner et al., 1972) then $\dot{M}_{1}=M_{1} / \tau_{p} \sim 2 \times 10^{19} \mathrm{gm} \mathrm{s}^{-1}$, whereas the oblique rotator model of Bath et al. (1974a) requires $\dot{M}_{1}^{p} \sim 10^{18} \mathrm{gm} \mathrm{s}^{-1}$.

In the case of recurrent novae we can estimate the mass lost from the system. Pottasch (1967) finds a mass $\sim 1 \times 10^{28} \mathrm{gm}$ for the shell ejected by RS Oph; as its outburst period is $\sim 30 \mathrm{yr}$, the current value of $-\dot{M}_{1}$ is $\sim 1 \times 10^{19} \mathrm{gm} \mathrm{s}^{-1}$. If the mass ejected results from accumulation of material onto the white dwarf, all of which is thrown off during an outburst, then this value of $\dot{M}_{1}$ is representative of the transfer rate.

We must also consider the mass flux $\dot{M}_{d}$ through the disc, keeping in mind that we have no a priori reason for supposing that the steady state condition $\dot{M}_{1}=\dot{M}_{d}=-\dot{M}_{2}$ holds: some mass may be lost from the system (splashed from the hot spot and lost from the edge of the disc as angular momentum is transferred outwards) and the disc may be increasing in mass. As we can only observe one side of the disc, its apparent luminosity $L_{d} \sim\left(G \dot{M}_{1} / 4 R_{1}\right) \dot{M}_{d}$ and the luminosity of the hot spot is $L_{s} \sim\left(G M_{1} / 2 r_{d}\right) \dot{M}_{2}$, hence 


$$
\begin{aligned}
\frac{L_{d}}{L_{s}} & \sim \frac{1}{2} \frac{r_{d}}{R_{2}} \frac{\dot{M}_{d}}{\left|\dot{M}_{2}\right|} \\
& \sim 15 \frac{\dot{M}_{d}}{\left|\dot{M}_{2}\right|}
\end{aligned}
$$

In the visible, most CV's do not show continua from their discs; those few that do are not seen preferentially at low inclination (e.g. UX UMa eclipses) so we cannot attribute visibility of discs to effects of inclination. Thus in the visible $L_{\mathrm{d}} \approx \frac{1}{5} L_{s}$, but this may not be representative of the bolometric luminosity. As can be seen from the above formula, satellite ultraviolet light curves of CV's, made in order to determine the relative contributions of the hot spot and disc, should provide a sensitive means of estimating $\dot{M}_{d}$. A limit of $\dot{M}_{d}<5 \times 10^{17} \mathrm{gm} \mathrm{s}^{-1}$ has already been set by the absence of X-rays from U Gem and RX And (Henry et al., 1975); if the value $-\dot{M}_{1} \sim 10^{18} \mathrm{gm} \mathrm{s}^{-1}$ given above is at all representative we should expect $L_{d} / L_{s} \gtrsim 30$ and there will not be a detectable hump in the ultraviolet.

In novae, it is obvious that the outer layers of the primary, and probably the disc, are lost to the system during an outburst. Pottasch (1959) finds a total nova shell mass $\sim 1-3 \times 10^{29} \mathrm{gm}$. Evidence for mass loss from dwarf novae is less easy to obtain; there are no doppler-shifted lines indicative of mass loss during an outburst. However, Robinson (1973d) has found evidence of material surrounding Z Cam which has been interpreted (Warner, 1974c) as arising from outbursts and amounting to $\sim 1.5 \times 10^{18} \mathrm{gm} \mathrm{s}^{-1}$; this amount of mass loss $\left(\sim 10^{-9} M_{\odot}\right.$ per outburst) would not show up in spectra of dwarf novae taken during outburst. It is possible, therefore, that most of the mass transferred to the disc between outbursts is lost to the system during outburst.

Possible evidence for circumstellar material near WZ Sge was given by Krzeminski and Smak (1971). However, the present writer feels that their suggested $\mathrm{K}$ line absorption has emission borders and thus originates in the disc. On the other hand Herbig's (1950) K line absorption in UZ Ser, seen at maximum, is probably interstellar: UZ Ser is comparatively faint at minimum $\left(m_{v}=16.6\right)$ and has low galactic latitude. If it has $M_{v}=+7.5$ the resulting distance of $600 \mathrm{pc}$ could give a $\mathrm{K}$ line equivalent width $\sim 200 \mathrm{~m} \AA$.

Evidence exists for a stream of material leaving the disc. In V Sge, Herbig et al. (1965) found $\mathrm{H}$ absorption reversals which, on integration of the velocity curve, was attributable to a stream of material emerging from the system. The slow recovery of brightness after eclipse in UX UMa (Warner and Nather, 1972c) and Z Cha (Warner, 1974a) might be associated with an absorbing stream. The spectra of RW Tri (Walker, 1971) show that the longward emission component is absent shortly after eclipse, as though the receding portion of the disc is obscured. A similar effect may exist in the spectra of VVPup (Warner and Nather, 1972a).

The hydrodynamical gas stream calculations of Sorensen et al. (1975) show that a stream of material leaving the disc can arise if the secondary has a small direct rotational velocity. Some evidence for non-synchronous rotation in the secondary of $\mathrm{ZCha}$ has been given by Warner (1974a).

One of the central problems in the theory of CV's is identification of the mechanism that drives the mass transfer. In the recurrent novae and GK Per we see evolved secondaries, and we may be fairly certain that the mechanism that started mass transfer is evolutionary expansion filling the Roche lobe. The masses of the secondaries (Table III) in the 
other systems are in general too small for evolutionary effects to be currently driving the mass transfer, although we cannot exclude the possibility that the secondaries were once considerably more massive and evolution started the process.

Faulkner (1971) has produced an elegant theory based on loss of angular momentum through the emission of gravitational radiation. An earlier discussion by Paczynski (1967) assumed degenerate secondaries, but Faulkner adopts main sequence structures for his secondaries. For the fully conservative case, and using the power radiated by a binary system (Landau and Lifshitz, 1962):

$$
\frac{\mathrm{d} E}{\mathrm{~d} t}=\frac{32}{3} \frac{G^{4}}{c^{5}} \frac{\left(M_{1}+M_{2}\right) M_{1}^{2} M_{2}^{2}}{a^{5}}
$$

Faulkner finds the following evolutionary equation:

$$
\frac{d}{\mathrm{~d} t}\left\{\frac{G^{\frac{1}{2}} M_{1} M_{2} a^{\frac{1}{2}}}{\left(M_{1}+M_{2}\right)^{\frac{1}{2}}}\right\}=\frac{32 G}{5 c^{5}}\left(\frac{M_{1} M_{2}}{M_{1}+M_{2}}\right\}^{2}\left(\frac{2 \pi}{P}\right)^{5} a^{4}
$$

the solution of which provides a timescale $T_{g}$ given by

$$
\begin{aligned}
T_{g} & \sim \frac{5 c^{5}}{32 G^{3}}\left(M_{1}+M_{2}\right) R_{\odot}^{4} M_{\odot}^{3}\left(M_{1}, M_{2} \text { in solar masses }\right) \\
& \sim 8.5 \times 10^{17}\left(M_{1}+M_{2}\right) \mathrm{s}
\end{aligned}
$$

Thus the average rate of mass transfer $-M_{2} \sim M_{\odot} / T_{g} \sim 5 \times 10^{15} \mathrm{gm} \mathrm{s}^{-1}$. This result applies even to the ultra-short period binary AM CVn (Faulkner et al., 1972). This is considerably below our observational estimates.

Mass loss from the CV system also carries away angular momentum, and allowance for this might be thought to speed up the transfer rate. We have solved the case with $M_{1}$ = constant and continuous, spherically symmetric mass loss equal to $\dot{M}_{2}$; the resulting $\dot{M}_{2}$ is not greatly different from that found by Faulkner.

We conclude that gravitational radiation is not the mechanism driving mass transfer in the CV's. There is, however, an area in which gravitational radiation may play a part. Prior to becoming CV's these binary systems presumably consist of a detached white dwarf - main sequence pair in a short period orbit. For systems with $M_{2}<1 M_{\odot}$ orbital shrinkage due to radiation of angular momentum may cause the secondary to fill its Roche lobe before it would do so by evolution. From Equation (14) and the virial theorem $E=-G M_{1} M_{2} / 2 a$, we have

$$
\frac{d}{\mathrm{~d} t}\left(\frac{1}{a}\right)=-\frac{64}{5} \frac{G^{3}}{c^{5}} \frac{\left(M_{1}+M_{2}\right) M_{1} M_{2}}{a^{5}}
$$

which has the solution

$$
t=\frac{5 c^{5}}{256 G^{3}} \frac{\left(a_{0}^{4}-a^{4}\right)}{M_{1} M_{2}\left(M_{1}+M_{2}\right)}
$$

where $a_{0}$ is the initial orbital separation.

As an example, we consider the case of $\mathrm{BD}+16^{\circ} 516$ (Young and Nelson, 1972) which has a $=2.21 \times 10^{11} \mathrm{~cm}, M_{1}=0.79 M_{\odot}, M_{2}=0.80 M_{\odot}$ and $R_{2}=0.85 R_{\odot}$. From $(2 \mathrm{~b})$ 
and (15) we find that the $\mathrm{K} 2 \mathrm{~V}$ secondary will fill its Roche lobe after $1.0 \times 10^{10} \mathrm{yr}$. Taking the lifetime $t_{m s}$ on the main sequence as $1.1 \times 10^{10} M_{2} / L_{2}$ we have $t_{m s} 3 \times 10^{10}$ $\mathrm{yr}$, so the initiating of mass transfer will more likely be a result of gravitational evolution in the case of this star.

\section{Outburst Light Curves}

11.1. NOVAE

A complete discussion of light curves of classical novae is beyond the scope of this review; extensive details may be found in Payne-Gaposchkin (1957). Important new information has been obtained by satellite observations in the ultraviolet and ground-based infrared and radio observations, which shows that the older studies based on the traditional optical region have given a rather misleading picture of nova outbursts.

Infrared observations of FH Ser (Nova Ser 1970), V1229 Aql (Nova Aql 1970) and HR Del (Nova Del 1967) in the region 1 to $25 \mu$ (Geisel et al., 1970) show that, during the fall in visual luminosity of novae from their maximum at $\sim 1-2$ days after outburst, the infrared luminosity increases over the first 90 days. At 90 days, the infrared luminosity is $\sim 5 \times 10^{4} L_{\odot}$ and corresponds to radiation from a circumstellar dust shell with a temperature of $900 \mathrm{~K}$. A mass of $\sim 10^{-6} M_{\odot}$ of dust is required to provide the observed luminosity, implying a total shell mass $\sim 10^{-4} M_{\odot}$. After infrared maximum, the luminosity decays with a half-life $\sim 100$ days.

Radio emission at 11,4 and $2 \mathrm{~cm}$ was detected from HR Del and FH Ser (Hjellming and Wade, 1970). The observations of FHSer, made 130-190 days after outburst, suggested an increasing radio luminosity during that period.

OAO-2 observations (Gallagher and Code, 1974) of FHSer were made over the first 58 days of the outburst. These showed that after visual maximum the peak of the energy distribution shifted to shorter wavelengths, being below $1900 \AA$ after 58 days. As a result, despite the fall in visual brightness, the nova maintained a constant luminosity $\sim 1.6$ $\times 10^{4} L_{\odot}$ over the time of observation. Combined with the infrared observations, a luminosity $\sim 7 \times 10^{4} L_{\odot}$ during the first 100 days seems likely, and hence the total radiated energy is $\sim 3 \times 10^{45} \mathrm{erg}$. The kinetic energy of the shell is also $\sim 10^{45} \mathrm{erg}$, but much of this may be radiated in the infrared by collisional heating of the interstellar gas.

Gallagher and Code speculated that the decline to visual minimum after a nova outburst may result solely from the redistribution of flux to shorter wavelengths, implying remnant temperatures $\sim 200000 \mathrm{~K}$. This has largely been disproved by the inability (Gallagher and Holm, 1974) of OAO-2 to detect FH Ser eighteen months after outburst, the non-detection of DQ Her and RS Oph and the observed flux distributions in RR Pic and V603 Aql discussed in Section 6.

After falling $\sim 3 \frac{1}{2} \mathrm{mag}$ from visual maximum, a nova enters the transition region. At this time many novae show oscillations with amplitudes up to two magnitudes. These oscillations may show considerable regularity as in GK Per and V603 Aql, but are in general quite erratic (as in V465 Cyg and DK Lac). The start of the transition region marks the time when the ejected circumstellar shell becomes optically thin, allowing the inner photosphere (formed by continuing mass ejection) to be seen. It is this photosphere that oscillates and it has been pointed out by Starrfield et al. (1974a) that the instability probably is the hydrodynamic one because the white dwarf at this stage of the outburst 
has a hot atmosphere reaching out to the Roche surface. If this is the case it is instructive to see how irregular a light curve such an instability produces; with the exception of GK Per and V603 Aql the transition oscillations resemble the peculiar light curve of AE Aqr (Zinner, 1938) rather than the more systematic variations of the dwarf novae (see below). We need also to explain why only some novae show oscillations; there does not seem to be any correlation with orbital size - e.g. GK Per $\left(P=16^{\text {h }} 26^{\mathrm{m}}\right)$ and V603 Aql $\left(3^{\mathrm{h}} 19^{\mathrm{m}}\right)$ showed oscillations, whereas T Aur $\left(4^{\mathrm{h}} 54^{\mathrm{m}}\right)$ and DQ Her $\left(4^{\mathrm{h}} 39^{\mathrm{m}}\right)$ did not. The oscillations in GK Per (Payne-Gaposchkin, 1957, Figure 4.15) were evident only a few days after maximum and have nearly constant amplitude in intensity for the next 100 days (growing in amplitude on a magnitude scale as the background from the ejected shell decays away).

A number of transient X-ray sources have been discovered, which may be related to nova outbursts. These are Cen X-2 (Chodil et al., 1968; Lewin et al., 1968), Cen X-4 (Conner et al., 1969), Cet X-2 (Harries et al., 1971) 2U 1543-47 (Matilsky et al., 1972) Cep X4 (Ulmer et al., 1973) and Ariel 1118-61 (Ives et al., 1975). Of these, only Cen X-2 and 2U 1543-47 have possible optical identifications.

The X-ray emission from these objects, after a sudden turn-on, generally remains high for a few weeks and then decays rapidly. The behaviour is remarkably similar to the light curves of the slower of the classical novae (e.g. DQ Her), in the case of Cen X-2 (Rao et al., 1969) even showing a possible recovery as in the DQHer light curve. X-ray spectra give temperatures $\sim 1-4 \times 10^{7} \mathrm{~K}$, which could be connected with the coronal lines observed in some recurrent novae.

However, Sofia (1972) has pointed out that if the transient X-ray sources are identified as novae, then total energies $\sim 10^{46}-10^{48} \mathrm{erg}$ are implied, which are difficult to explain in terms of currently suggested nova mechanisms. Sofia prefers type II supernovae of extragalactic origin as a possible source. Silk (1973) finds, however, that the transient sources are more likely to be halo objects with $L \sim 4 \times 10^{38} \mathrm{erg} \mathrm{s}^{-1}$, giving a total energy output $\sim 10^{45} \mathrm{erg}$. On energetic grounds, therefore, we cannot dismiss novae as being unconnected with the transient X-ray sources. The occurrence rates are also not too dissimilar: $12 \mathrm{yr}^{-1}$ for the transient sources (Silk, 1973) compared with $40 \mathrm{yr}^{-1}$ for novae (Allen, 1973).

The discovery of a period of $6.75 \mathrm{~min}$ in the source Ariel 1118-61 (Ives et al., 1975), and the suggested interpretation (Pringle and Webbink, 1975) in terms of an ultra-short period interacting binary, brings this source into the general domain of cataclysmic variables, but the explanation of the outburst given by Pringle and Webbink is not related to the normal nova mechanism, being in fact a result of a reduction in rate of mass transfer.

Until a definite connection is made between an observed nova outburst and a transient $\mathrm{X}$-ray source, we must consider their possible relationship as unproven.

\subsection{RECURRENT NOVAE}

In Section 6 we saw that it is the presence of giant secondaries that give the recurrent novae their lower amplitudes. The total energy released during outbursts is therefore similar to ordinary novae of the same speed classes. A summary of the properties of the known recurrent novae is given in Table VII; $t_{2}$ is the time from outburst until the nova had fallen two magnitudes below maximum. As can be seen some outbursts are very fast 
TABLE VII

Recurrent novae

\begin{tabular}{|c|c|c|c|c|c|}
\hline & & $m_{v}(\max )$ & $m_{v}(\min )$ & $\Delta m_{v}$ & $t_{2}$ \\
\hline VY Aqr & $\begin{array}{l}1907 \\
1962\end{array}$ & $\begin{array}{l}8.0: \\
9.0:\end{array}$ & $16.6^{\mathrm{a}}$ & $\begin{array}{l}\text { 8.6: } \\
7.6:\end{array}$ & $\begin{array}{l}6: \\
5\end{array}$ \\
\hline $\mathrm{T}$ CrB & $\begin{array}{l}1866 \\
1946\end{array}$ & $\begin{array}{l}2.0 \\
2.0\end{array}$ & 10.5 & $\begin{array}{l}8.5 \\
8.5\end{array}$ & $\begin{array}{l}5 \\
5\end{array}$ \\
\hline RS Oph & $\begin{array}{l}1898 \\
1933 \\
1958 \\
1967\end{array}$ & $\begin{array}{l}4.3 \\
4.3 \\
5.0: \\
4.9\end{array}$ & 12.3 & $\begin{array}{l}8.0 \\
8.0 \\
7.3: \\
7.4\end{array}$ & $\begin{array}{l}4 \\
4 \\
6 \\
7\end{array}$ \\
\hline T Pyx & $\begin{array}{l}1890 \\
1902 \\
1920 \\
1944 \\
1966\end{array}$ & $\begin{array}{l}7.9: \\
7.3 \\
6.6 \\
7.1 \\
7.7\end{array}$ & 14.5 & $\begin{array}{l}6.6: \\
7.2 \\
7.9 \\
7.4 \\
6.8\end{array}$ & $\begin{array}{c}100: \\
100: \\
100: \\
100: \\
80\end{array}$ \\
\hline V1017 Sgr & $\begin{array}{l}1901 \\
1919 \\
1973\end{array}$ & $\begin{array}{r}10.8 \\
7.2 \\
10.2\end{array}$ & 14.4 & $\begin{array}{l}3.6 \\
7.2 \\
4.2\end{array}$ & $\begin{array}{r}200: \\
90 \\
200:\end{array}$ \\
\hline U Sco & $\begin{array}{l}1863 \\
1906 \\
1936\end{array}$ & $\begin{array}{l}9.1 \\
8.8 \\
8.8\end{array}$ & $>17.6^{a}$ & $\begin{array}{l}>8.5 \\
>8.8 \\
>8.8\end{array}$ & $\begin{array}{l}2 \\
2 \\
1\end{array}$ \\
\hline
\end{tabular}

${ }^{\mathrm{a}} m_{\mathrm{pg}}(\mathrm{min})$

and it is possible that other outbursts may have been missed. In the case of V1017 Sgr two fast and one slow outburst have been observed. The existence of both types in one object should be taken note of for the theory of nova outbursts.

The similarities, especially in the case of T Pyx, with ordinary novae suggest that we are looking at the short-period tail of a distribution among the novae; all novae may be recurrent. However, the occurrence of giant secondaries in the known recurrent novae may be influential in their recurrence: giant secondaries are otherwise known or suspected only in the peculiar slow novae RR Tel and RT Ser. Nevertheless, a close watch should be kept on those novae known to have low amplitudes in the expectation of detecting further outbursts. Possible candidates are WX Cet $\left(1963\right.$, range $\left.7^{m} .5\right)$, HR Lyr $(1919,8.9)$, IM Nor (1920, 7.5), FM Sgr (1926, 8.3), HS Sgr (1900, 6.5), V441 Sgr (1930, 7.8), V1016 Sgr $(1899,8.0)$ and EU Sct $(1949,8.6)$.

\subsection{DWARF NOVAE}

The outburst light curves of dwarf novae possess a number of systematic features that are of the greatest importance in providing clues to the nature of the outbursts. These are (a) existence of different widths and amplitudes of outburst, with statistical relationships between these and the intervals between them, (b) existence of supermaxima, (c) the standstills of Z Cam stars and (d) a possible period-amplitude relationship.

The statistical studies of SS Cyg by Sterne and Cambell (1934) and of U Gem by Greep 
(1942) show that bright maxima are usually followed by an unusually long interval before the next outburst, and this outburst is likely to be smaller than usual. The impression that one obtains from this correlation is that there is a constant source of energy available for the outbursts, but not all the accumulated energy is necessarily released during each outburst.

There is a wide spectrum of outburst light curves among the dwarf novae. Some show quite similar outbursts on every occasion; others, such as SSCyg, show roughly alternating wide and narrow maxima with the widths being only $\sim 30 \%$ different. There is then a sequence of behaviour which extends up to objects having normal maxima occasionally interspersed by supermaxima in which the star briefly reaches $0^{m} .5-0^{m} .7$ brighter than normal and then remains at normal maximum brightness for as much as five times longer than in an ordinary maximum. In those stars with well-defined supermaxima (e.g. WX Hyi, VW Hyi, Z Cha, SU UMa, AY Lyr) the supermaxima recur with a regularity that appears more reliable than that of the normal outbursts. Table VIII lists the average outburst recurrence periods $P_{n}$ and $P_{s}$ and the durations $D_{n}$ and $D_{s}$ for normal maxima and supermaxima of the few stars that have been studied adequately. The ratio $s$ of the average energy released in supermaxima and normal maxima will crudely be given by

$$
s=\left(\frac{D_{s}}{D_{n}}\right)\left(\frac{P_{n}}{P_{s}}\right)
$$

It can be seen from Table VIII that $s$ is probably not significantly different from unity, implying that the same energy source is being utilised in supermaxima as in normal maxima.

The extensive set of data on VWHyi compiled by F. M. Bateson indicate that the timings of normal and supermaxima are independent. Although there is a tendency for supermaxima to be both preceded and followed by unusually long intervals at minimum light, there are many instances of supermaxima starting while a normal outburst is in progress. Figure 3 illustrates such a case. The dip between the normal outburst curve and the onset of the supermaximum can be as little as one magnitude or as deep as normal minimum light.

Standstills of the Z Cam subclass of dwarf novae occur at irregular intervals. They always follow a normal outburst of the star and result in the star remaining about one magnitude below maximum light for intervals ranging from just a few days to as long as several years. Almost without exception, a standstill is followed by a return to minimum light. From the point of view of energy supply there are two very important properties of standstills: in a given star they always occur at very closely the same apparent magnitude, and in the case of Z Cam it has been established (Lortet, 1968) that the energy

TABLE VIII

Outburst periodicities in dwarf novae with supermaxima

\begin{tabular}{lccccl}
\hline & $P_{n}$ & $P_{s}$ & $D_{n}$ & $D_{s}$ & $s$ \\
\hline VW Hyi & 28 & 179 & 4 & 17 & 0.7 \\
Z Cha & 96 & 313 & 3 & 10 & 1.0 \\
SU UMa & 17 & 170 & 2 & 13 & 0.7 \\
AY Lyr & 31 & 200 & 4 & 16 & 0.6 \\
\hline
\end{tabular}




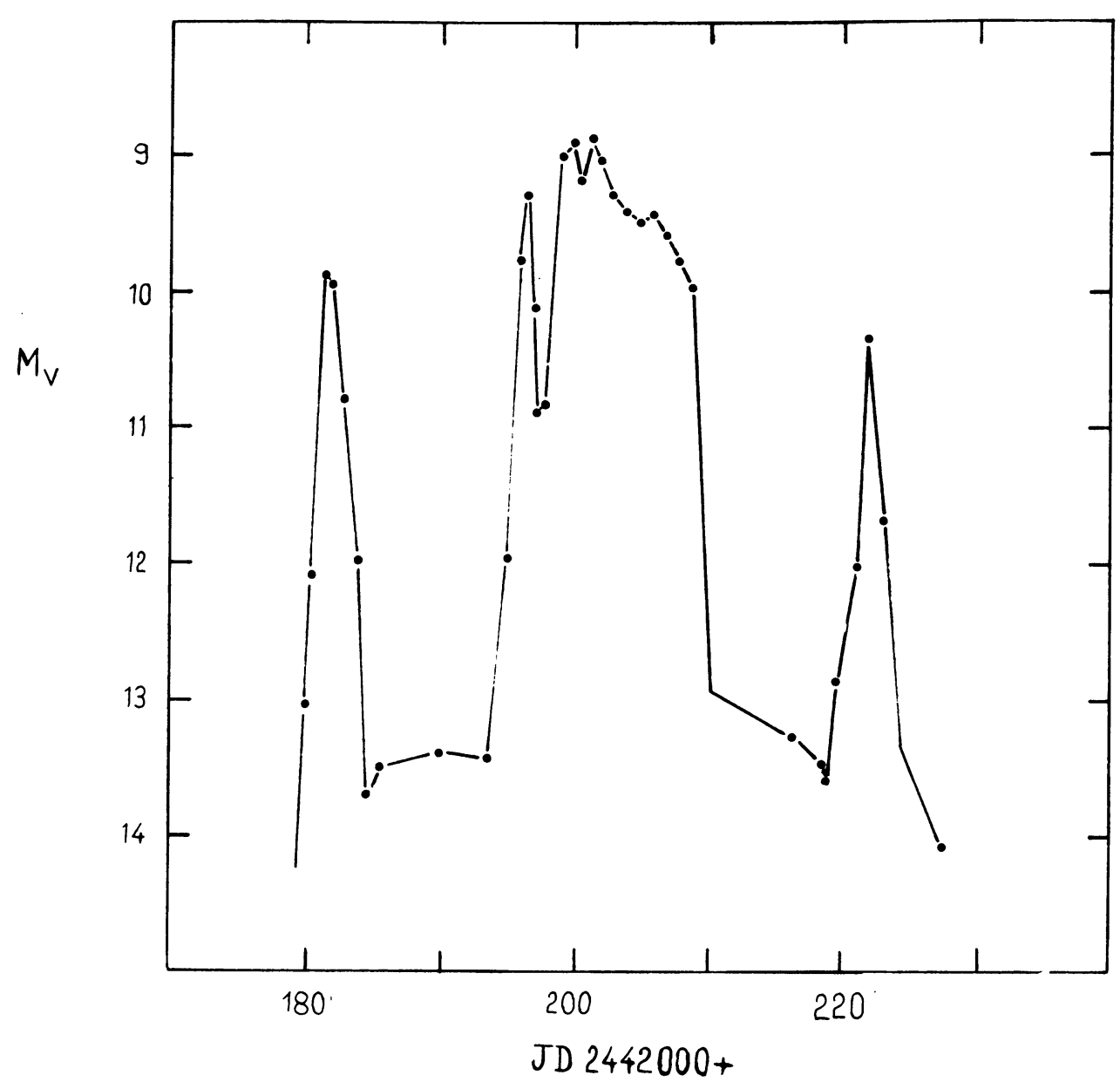

Fig. 3. Maxima and supermaxima of VW Hyd.

radiated in the $U B V$ bands during standstill is the same as the rate of emission averaged over the normal light curve (i.e. including outbursts and the intervals of quiescence) when standstills are absent. This latter result should be checked for other Z Cam stars.

Both of the above results are in agreement with our thesis that there is a constant source of energy available for outbursts - during standstills this supply is used continually instead of being stored.

From inspection of published light curves, and discussions with F. M. Bateson, it appears that all of the dwarf novae with short recurrence periods $\left(P_{n} \lesssim 30^{d}\right)$ fall either in to the $\mathrm{ZCam}$ subclass or show supermaxima. These two subclasses are mutually exclusive. The stars with the smallest $P_{n}$ show the most regular supermaxima. Those short period stars not yet known to fall into these subclasses are poorly observed. The probability of a star showing standstills or supermaxima decreases rapidly for $P_{n}>30^{\text {d }}$.

The period-amplitude relationship for CV's (Kukarkin and Parenago, 1934; Payne- 
Gaposchkin, 1957) shows that outbursts are more energetic the longer the interval between them. This relationship needs a complete reinvestigation, allowing for aspect effects in the disc of the dwarf and recurrent novae and the influence of the secondary on $M_{\nu}$; and preferably a relationship involving total energy emitted rather than amplitude should be sought. For example, two of the three outbursts of V1017 Sgr (Table VII) had amplitude $\sim 4^{m}$ but lasted for several hundred days; the other outburst had $\sim 7^{m}$ amplitude but was of much shorter duration. The total energy emitted in the three cases may have been the same. We must also recognise that the rate of mass transfer may be different in the recurrent and dwarf novae, in which case we must not expect the same relationship to hold for the two classes of star. However, the general form of the periodamplitude relationship is in accord with the view that the average energy emitted is approximately the same for all CV's.

In Section 6 we concluded that the average luminosity of dwarf novae at maximum is $16 L_{\odot}$. The duration of outburst is usually 4-8 days, but most dwarf novae are within half a magnitude of maximum for only 2-3 days. The total energy radiated is therefore $\sim 1 \times 10^{40} \mathrm{erg}$. In individual cases, however, this average may be greatly exceeded. For a star with $M_{v}=7.5$ at minimum, amplitude of outburst $5 \mathrm{mag}$ and effective duration 2 days, we have a total of $\sim 2 \times 10^{40} \mathrm{erg}$ radiated. During a supermaximum, where the system stays near maximum for $\sim 15$ days, up to $10^{41}$ erg may be radiated. The positive detection of soft X-rays from SSCyg by Rappaport et al. (1974) was made during an outburst of that object. However, if the outburst mechanism is the source of $\mathrm{X}$-rays we will not have seriously underestimated the luminosity during outburst. Rappaport et al. find $\sim 0.4 L_{\odot}$ in soft X-rays from SSCyg for an assumed distance of $100 \mathrm{pc}$; our discussion of the distance to SS Cyg in Section 6 indicates that this luminosity may have to be increased by a few times but the optical luminosity still dominates. Evidently the high temperature region is small compared to the size of the disc.

\subsection{SYMBIOTIC VARIABLES}

Although we have largely ignored the symbiotic variables in this review, their structural similarity to the recurrent novae demands a comment here. Payne-Gaposchkin (1957) states that of those stars possessing combination spectra, only those with M-type absorption spectra show light variations similar to those of the novae, although not all of the M-type objects show such large light variations. The M-type systems are in general long period variables with periods between 250 and 1000 days. In addition, major outbursts can occur. It is possible to separate the contributions from the red and blue components. Two examples will suffice to illustrate the general trends. In $Z$ And (Payne-Gaposchkin, 1946) the red component increases by $\sim 1$ mag during the $\sim 6$ mag outburst of the blue component. Spectroscopically it is evident that only a single shell is ejected, unlike the multiple shells or continuous ejection in classical novae. In R Aqr (Payne-Gaposchkin and Boyd, 1946) when the blue component was brightest the red star fell in brightness by $\sim 2$ mag and took two years to recover. A compilation of light curves of symbiotic variables has been given by Boyarchuk (1969).

It is tempting to consider the symbiotic variables as semi-detached (or at least nearly lobe-filling) systems in which the long-period variability of the red component modulates mass transfer to the blue star and may cause it (or a surrounding disc) to outburst. There is, however, no spectroscopic evidence for a disc in these systems. Also Walker (1957) 
found no significant flickering in four northern hemisphere symbiotics, a result that has been confirmed by a study of several southern hemisphere objects (A. R. Walker, private communication). This result contrasts with $\mathrm{T} \mathrm{CrB}$, which has very slow flickering activity. A possible explanation of the absence of a hot spot is that mass loss from the red star takes place as a stellar wind, rather than as a stream through the inner Lagrangian point, and as a result accretion onto the blue component is quasi-spherical rather than disc-like.

\section{Short Period Oscillations}

The 71-s coherent oscillations in brightness of DQ Her (visible amid the general flickering of this nova remnant), discovered by Walker (1954), initiated a search for coherent short period variations in other stars. Until the late 1960's this search was made using conventional photometric techniques; the flicker and scintillation noise associated with studies of faint CV's prevented any new discoveries. Using the improved techniques of pulsecounting photometry and the relative ease of handling the digitised data resulted in the discovery of rapid pulsators among the white dwarfs (Lasker and Hesser, 1969, 1971). These observations, by the Princeton group, failed to find any new, long-lived coherent oscillations in the CV's, although some possible periodicities were observed in $\mathrm{TCrB}$ (Lawrence et al., 1967) which have not subsequently been seen (Warner and Robinson, 1972b).

The extensive high speed photometric studies of CV's started at McDonald Observatory in 1968 (Warner, 1971; Nather and Warner, 1969, 1971) provided a wealth of material that could be searched for periodicities. On running this material through a power spectrum program success was almost immediate and resulted in the discovery that the dwarf novae show rapid oscillations of low amplitude during their outbursts (Warner and Robinson, $1972 \mathrm{~b}$ ). Since that time much of the study of periodicities in the CV's has concentrated on the erupting dwarf novae. However, periodicities in some other objects have been found. The current situation is summarised in Table IX.

\subsection{DWARF NOVAE}

Nine dwarf novae have now been found to have rapid oscillations during outbursts. Of these, six are ZCam stars and three are UGem types. Selection favours observation of outbursts of Z Cam stars because of their relatively greater frequency. So far no high speed photometric observations of the two brightest northern hemisphere objects U Gem and SS Cyg - have been made during outburst.

There is extensive observational evidence (Warner and Robinson, 1972b; Robinson, 1973b; Warner and Brickhill, 1974) that oscillations rarely occur on the rising branch of the outburst. The exception is in CN Ori when it was only $1 \mathrm{mag}$ above minimum light (Warner and Brickhill, 1975). In general the oscillations are observed shortly after maximum light. An exception is VW Hyi which, both at normal maxima and supermaxima, oscillates well down the recovery part of its outburst light curve (Warner and Brickhill, 1975). Among the dwarf novae periods from 16 to $34 \mathrm{~s}$ have been observed, with the range 24-28s being most favoured. The objects showing these periods have orbital periods from $89 \mathrm{~min}$ to $7 \mathrm{~h}$ and outburst time-scales ranging from 10 to 96 days. In three cases (Z Cam, AHHer and VW Hyi) oscillations have been detected in different outbursts; in all cases (Table IX) the periods were similar. 
TABLE IX

Periodicities in cataclysmic variables

\begin{tabular}{lllll}
\hline Name & Type & $P_{n}(\mathrm{~d})$ & Periods $(\mathrm{sec})$ & Ref. \\
\hline Z Cam & DN & 20 & $16.01,16.92,18.83$ & 1,2 \\
V436 Cen & DN & 25 & $20.0,20.5$ & 7 \\
SY Cnc & DN & 27 & 24.623 & 3 \\
CN Ori & DN & 19 & $25.00-24.27,33$ & 1 \\
RR Pic & N & & $26.2,28.7,36.9$ & 10 \\
KT Per & DN & 12 & $26.825-26.730$ & 11 \\
YZ Cnc & DN & 10 & 26.99 & 4 \\
Z Cha & DN & 96 & 27.67 & 5,6 \\
UX UMa & NL & & $28.54-30.03$ & 7 \\
VW Hyi & DN & 28 & $28-35$ & $7,8,9$ \\
CD -42 & & & \\
AH Her & NL & & $18.50,29.08,30.15,32.0$ & 1,7 \\
DQ Her & DN & 20 & $31.55,34.8$ & 12,13 \\
AM CVn & N & & 71.06 & 14 \\
\hline
\end{tabular}

\section{References}

1. Warner and Robinson (1972b)

2. Robinson (1973a)

3. Robinson (1973c)

4. Warner (1974a)

5. Warner and Nather (1972c)

6. Nather and Robinson (1974)

7. Warner and Brickhill $(1974,1975)$
8. Warner (1973a)

9. Hesser et al. (1974)

10. Warner (1975c)

11. Moffet and Barnes (1974)

12. Walker (1954)

13. Warner et al. (1972)

14. Warner and Robinson (1972a)

The observations, although extensive, are still far from sufficient to show exactly when the oscillations start and finish, or whether they are always present (at least for part of the time) at each outburst. Robinson (1973c) failed to find any oscillations during a low amplitude outburst of RX And. The writer failed to detect any on the night of maximum (and the following night) of V442 Cen in June 1973. These observations do not necessarily imply a complete lack of oscillations during outburst: the 27-s oscillations seen in Z Cha (Warner, 1974a) died out for a time during the observing run and a shorter run may not have detected them. In only two cases (Z Cam: Robinson, 1973c; V436 Cen: Warner, 1975b) have the oscillations during outburst been detected on more than one night. Their duration is therefore often less than $\sim 18 \mathrm{~h}$.

In all the studies so far made, power spectra and mean light curves show the oscillations to be pure sinusoids, within the error of observation. In those stars with the largest amplitude of oscillation (VWHyi, SY Cnc, V436 Cen) the power at the fundamental frequency is often over one hundred times the mean power at neighbouring frequencies, whereas the first harmonic contains no excess power greater than $\sim 50 \%$ of the local noise (see e.g. Robinson, 1973c, Figure 4).

Oscillation amplitudes from 0.0009 to 0.01 mag have been found, but these are correlated with apparent magnitude in such a way that, at least when oscillating with maximum amplitude, it is probable that the intrinsic amplitudes are almost the same (Warner, 1974a; Warner and Brickhill, 1975).

The discussion by Warner and Brickhill (1975) has shown that a range of behaviour exists for the oscillations during outbursts. Correlations of oscillation period with orbital 
phase, modulation of the rapid oscillations by longer periods, and phase shifts during the eclipses of ZCha have all been found. Rapid variations in amplitude cause confusion in the periodograms and may simulate apparent multiple periodicities. The most important conclusions are firstly that the phase shifts during eclipse imply that the entire disc is involved with the oscillations, and secondly that there is a good correlation between brightness and oscillation period: as a system brightens its period gets shorter and vice versa.

\subsection{NOVAE}

Until recently DQHer, the remnant of Nova Herculis 1934, was the only classical nova showing a periodicity, and a number of studies of it using increasingly sophisticated equipment and analysis techniques have been made. During studies of the 1925 nova remnant RR Pic, however, the author has recently found one occasion on which coherent oscillations, with several periods simultaneously present, occurred (Table IX). Other runs on the same object definitely did not contain periodicities. We therefore find that, as with the dwarf novae and nova-like variables, nova remnants may have oscillations with amplitudes only occasionally reaching detectability.

The DQHer 71-sec oscillations are present at all times, although there is some evidence that the amplitude may vary on a timescale of months or years. Walker's early studies (Walker, 1954, 1956, 1958, 1961) showed that the oscillations had constant period and their intrinsic amplitude is the same in all colours. The variation in amplitude around the orbit found by Walker (1958) has been reinvestigated by Warner et al.(1972) and confirms Walker's finding that the maximum amplitude is achieved shortly after eclipse. Walker, using chart recorder techniques, thought that the oscillations disappear completely for $\sim 20$ min centred on primary eclipse, but Warner et al. (1972) found that at most 5 cycles are missing during eclipse.

Mean light curves (Nather and Warner, 1969) indicated that the 71-s light curve is sinusoidal, and this was supported by power spectrum analysis (Warner and Robinson, 1972b). A more recent analysis by Nelson (1975) has claimed the presence of a subharmonic at $142 \mathrm{~s}$, but the power spectral analysis by Kiplinger and Nather (1975), based on superior observations, shows no significant power at $142 \mathrm{~s}$. Their analysis does, however, indicate a small excess of power at the first harmonic which if real (note that there are other probably spurious features of similar amplitude in the power spectrum) implies a harmonic amplitude $\sim 4 \%$ of the fundamental.

Swedlund et al. (1974) and Kemp et al. (1974) have claimed circular and linear polarisation to be present in DQHer amounting to $0.2 \%$ and $0.6 \%$ respectively in the ultraviolet, synchronous with the 71-s oscillations but with fundamental period $142 \mathrm{~s}$. These observations would imply that the oscillating component is highly polarised, but the absence of any 142-s component in the work of Kiplinger and Nather contradicts the polarisation measurements so further discussion should await confirmation of the latter.

The most important clue to the nature of the oscillations resides in the phase shift seen throughout the eclipse (Warner et al., 1972). The steady phase shift, totalling $360^{\circ}$, starting at the beginning of eclipse and continuing until the end shows that the entire disc around the primary in some way participates in the oscillations.

Herbst and Ostriker (1971) found that the 71-s period is getting shorter, this was confirmed by Warner et al. (1972) and has been further discussed by Herbst et al. (1974). 
The latter authors find a change in period of $-2.69 \times 10^{-5} \mathrm{~s} \mathrm{yr}^{-1}$ adequately fits all the data; but Nelson (1975) has claimed evidence for a non-linear decrease in period.

\subsection{NOVA-LIKE VARIABLES}

CD $-42^{\circ} 14462$, the nova-like variable discussed in Section 7 , showed strong oscillations (amplitude $0.003 \mathrm{mag}$ ) with a period of $29.04 \mathrm{~s}$ in April 1973 (Warner, 1973a). Analysis of observations made in August 1971, April and May, 1972 showed the presence of persistent low amplitude $(0.00028 \mathrm{mag})$ oscillations throughout that time, with periods of 29.08 and $30.15 \mathrm{~s}$ occurring simultaneously (Hesser et al., 1974). More recent studies (Warner, 1975c) show occasional presence of periodicities, with $18.50 \mathrm{~s}$ on one occasion and $32.0 \mathrm{~s}$ on two consecutive nights.

UX UMa was found to possess a periodicity near 29s (Warner and Nather, 1972c) which subsequently has been found to be possibly changing cyclically with a period of weeks (Nather and Robinson, 1974). The oscillation period is sufficiently constant during one orbit for it to be used to investigate the phase shift during eclipse and (Nather and Robinson, 1974) a $360^{\circ}$ phase shift is found, but in the opposite sense to that for DQ Her, again starting and ending with the eclipse.

\subsection{DISCUSSION}

The observed periods, interpreted as gravitationally controlled phenomena, imply mean densities $\bar{\rho} \sim\left(G p^{2}\right) \sim 2 \times 10^{4} \mathrm{gm} \mathrm{cm}^{-3}$ which in turn implies a source in the vicinity of the degenerate primary. The limited range of periods, and their preference for values near $26 \mathrm{~s}$, signifies a uniformity in structure that is not shared by the other more obvious systemic properties, although the uncertainties in Table III are such that we cannot exclude the possibility that the range of primary masses may be very small. U Gem, which certainly appears to have a lower mass primary, should be searched for oscillations during outburst. The consistency of periods at different outbursts of the same dwarf nova also points to a uniformity of behaviour (independent of whether the outburst is a maximum or a supermaximum in VW Hyi) as does the presence of oscillations in dwarf novae only when they are passing through a definite range of brightness.

Despite the evidence that the oscillations originate near the primary, the phase shifts seen through eclipse (now detected in a nova remnant, a nova-like variable, and a dwarf nova) show that the whole disc is somehow involved. The oscillation period of the disc itself will be of the same order as the rotation period, which is $\sim 1000 \mathrm{~s}$ for the outer disc regions of systems with $P \sim 5 \mathrm{~h}$; we must therefore look elsewhere for the explanation of the involvement of the disc. Herbst et al. (1974) suggest the propagation of waves in a spiral pattern through the disc; Nather and Robinson (1974) and Warner and Brickhill (1975) have suggested reflection off the disc. Detailed models of these mechanisms have not yet been worked out.

The origin of the oscillations is not yet clear. Bath (1973) has proposed that they arise from eclipses of transient hot spots formed at the inner edge of the accretion disc. For primary masses near $1 M_{\odot}$ the Keplerian velocity is $\sim 20 \mathrm{~s}$. Bath et al. (1974b) estimate coherence times for the oscillations, based on the time taken to shear the spot by differential rotation of the disc, to be $\sim 2 \times 10^{3} \mathrm{~s}$. The observations by Warner and Brickhill (1975), however, show that the oscillations maintain coherence for at least $2 \times 10^{4} \mathrm{~s}$. The decrease in period during rising light and increase during falling light in dwarf novae 
would imply spots drifting first inwards and then outwards in the disc. The highly sinusoidal nature of the oscillations, and the maintenance of this shape even when large phase shifts are occurring during eclipse (Nather and Robinson, 1974), argues against a simple spot model.

Warner and Robinson (1972b) suggested non-radial pulsations of the white dwarf primaries, excited by the outburst in the case of the dwarf novae, as the origin of the oscillations. In the light of the study of non-radial modes of white dwarfs by Brickhill (1975) the $g$-modes are the indicated choice: the excitation energies are sufficiently low and the periods of the low-order modes fall in the correct range. Furthermore, the eigenfunctions have large amplitude only in the outer layers, which are likely to be highly perturbed during outbursts. Although the calculations show that the periods of $g$-modes do indeed increase with decreasing luminosity, as is observed in the dwarf novae, these results cannot be directly applied to the observations because the equilibrium models from which they were calculated differ in internal energy by $\sim 10^{45}$ erg for eigenvalues differing by several seconds, whereas in dwarf novae only $\sim 10^{40}$ erg are liberated during the whole outburst. It is more likely that the changing periods result from large temperature changes in the outer layers during outburst, caused, for example, by a higher rate of accretion (Section 13). The observed limited range of luminosity during which oscillations are present may be a result of the need for at least some lower limit on the accretion rate in order to excite the oscillations, and choking or obscuration of the oscillations if the accretion rate is too high. Such variations in accretion rate may also explain why in CD $-42^{\circ} 14462$ and UX UMa the oscillations are not always present.

In DQ Her, however, the coherence and presence of the oscillations over $20 \mathrm{yr}$ should caution us that their origin may be slightly different. Bath et al. (1974a) have proposed an oblique magnetic rotator model for DQ Her. Although such a model does not appear applicable to the other systems, in which large period changes are seen, we must also recognise that the oscillation period in DQ Her is twice that observed in the other systems (with the exception of AM CVn, which is itself a unique system: (Faulkner et al., 1972)).

If the oscillations are arising from hot spots near the inner edge of the accretion disc, or on the surface of the white dwarf itself, then the models of accretion discs (Pringle and Rees, 1972) indicate that they will occur in regions of extremely high temperature. Satellite observations in the far ultraviolet, made with sufficient time resolution, may therefore show a considerably greater amplitude for the rapid oscillations.

\section{Origin of the Outbursts}

The presence of a degenerate dwarf in the cataclysmic variables provides, at one and the same time, a source of energy for the outbursts, and a source of ambiguity. Kraft (1962), following Mestel's (1952) work on the structure of white dwarf stars, pointed out that accretion of hydrogen-rich material onto the surface of a white dwarf could lead to a thermonuclear runaway at the edge of the degenerate core. Although Kraft (1964a) later changed his mind because he suspected that any energy produced in this manner would be conducted away into the degenerate core, the work of Saslaw (1968) and later studies have shown that the energy production is not in a region of very high conductivity. Thus the special equation of state for white dwarf interiors leads to one possible energetic mechanism, and the accumulation time provides a possible explanation of the range of times between outbursts. 
On the other hand, the deep potential well of a white dwarf suggests that energy may be released through infall of matter to the primary. Two possibilities have been suggested for modulation of this infall: hydrodynamic instability of the mass transfer from the secondary (Bath, 1973) or instabilities in the disc (Smak, 1971c; Osaki, 1974).

In the case of the classical novae, only nuclear energy sources appear sufficient to account for the outbursts. In hydrogen burning, $6 \times 10^{18} \mathrm{erg} \mathrm{gm}^{-1}$ are available so a nova explosion must consume $\sim 5 \times 10^{26} \mathrm{gm} \mathrm{s}$, i.e. about $2 \times 10^{-3}$ of the mass ejected. Detailed calculations (Starrfield et al., 1974b and references cited therein) show reasonable agreement with light curves and element abundances for novae.

Despite an initial flurry of indecision about which, if any, of the components is responsible for outbursts in the dwarf novae, the observations now prove decisively that the outburst takes place in the white dwarf or its surrounding disc. The indecision arose after Krzeminski (1965) interpreted his important observations of U Gem during outburst as indicating a brightening of the secondary. The radial velocity observations of SSCyg made during outburst by Walker and Chincarini (1968) apparently showed that the primary is the centre of the outburst, but Smak (1969) advised caution because the solution for the radial velocity curve was not unique. The later observations by Walker and Reagan (1971) have cleared up this point in favour of the primary. The hot spot model of U Gem, proposed independently by Smak (1971a) and Warner and Nather (1971) has enabled reinterpretation of Krzeminski's observations in terms of a brightening centred on the primary. There is room, however, for more observations on this point. The orbital period of U Gem is now very well determined and radial velocities should be measured during outbursts. The radial velocity curve of EM Cyg (Robinson, 1974) could provide a check for this system if velocities are obtained during the relatively frequent outbursts of this object. Further photometric and spectroscopic observations made during outbursts of the eclipsing dwarf novae (BV Cen, Z Cha, EM Cyg, U Gem, EX Hya) should be made in order to study the temporal variations in brightness distribution across the discs of these stars.

The origin of the outbursts in dwarf novae is still controversial. One view, using the principle of Occam's razor, is that all nova outbursts have a common origin; then, having established that classical novae are thermonuclear events, the method of infinite descent establishes dwarf novae also to be powered by the atom. This attitude, however, is to some extent influenced by the supposed smooth transition in timescales in the sequence classical novae - recurrent novae - dwarf novae. Our discussion in Section 6 tends to break this sequence by positing that only the recurrent novae, and some unusually slow novae, have giant secondaries, thus relating them more closely to the symbiotic variables than to novae proper.

Calculations (Starrfield et al., 1974b) of white dwarfs with $M<0.5 M_{\odot}$, or with low CNO enhancements, are able to produce expansions of the outer layers which are of too low velocity to eject matter, but which have a kinetic energy similar to the total energy liberated in a dwarf nova outburst: the kinetic energy is converted when the expanding layers interact with the disc. The argument against this mechanism is that $\sim 10^{44}$ erg must be injected into the outer (non degenerate) layers in order to cause significant expansion; even at $100 L_{\odot}$ it would take $15 \mathrm{y}$ to radiate this away, but dwarf novae are observed to decline in only a few days. If the outbursts are thermonuclear in origin, then mass must be consumed at an average rate of $\sim 1 \times 10^{15} \mathrm{gm} \mathrm{s}^{-1}$. We do not know what fraction of 
the mass accreted is consumed; if it is the same as in the classical novae (see above) then $\dot{M}_{1} \sim 1 \times 10^{18} \mathrm{gm} \mathrm{s}^{-1}$ is required.

On the other hand, if it is gravitational energy that is being liberated both during outbursts and during the quiescent stage, we have $\bar{L} \sim G M_{1} R_{1}^{-1} \dot{M}_{d}$. The rate of energy loss from a dwarf nova, averaged over the outbursts, is

$$
\begin{aligned}
\bar{L} & \sim L_{\min }\left[1+\frac{L_{\text {out }}}{L_{\min }} \frac{D_{\mathrm{n}}}{P_{n}}\right] \\
& \sim 5 L_{\min } .
\end{aligned}
$$

This result will be insensitive to $P_{n}$ since $L_{\min }$ and $D_{n}$ are roughly constant and $L_{\text {out }}$ $\propto P_{n}^{\beta}$ where $\beta$ differs little from unity as a result of the Parenago-Kukarkin relationship. This result agrees with an estimate given by Zuckerman (1954). Thus $\dot{M}_{d} \sim 5 \times 10^{16} \mathrm{gm} \mathrm{s}^{-1}$ on average, with peak values $\sim 2 \times 10^{18} \mathrm{gm} \mathrm{s}^{-1}$ during outbursts. The total mass lost from the disc to the primary is therefore $\sim 2 \times 10^{-9} M_{\odot}$ for a normal maximum and $\sim 10^{-8} M_{\odot}$ during a supermaximum. These figures at the present time appear consistent with what is known of the structure of dwarf novae.

\section{Acknowledgements}

The author has benefited from discussions and correspondence with F. M. Bateson, A. J. Brickhill and E. L. Robinson.

\section{References}

Adams, W. S. and Joy, A. H.: 1922, Pop. Astr. 30, 103.

Allen, C. W.: 1973, in Astrophysical Quantities, 3rd edition, Athlone Press, London.

Baade, W. and Humason, M. L.: 1943, Publ. Astron. Soc. Pacific 55, 260.

Bailey, J.: 1975, J. British Astron. Assoc. 85, 217.

Bateson, F. M.: 1973, Roy. Astron. Soc. New Zealand Var. Star Sec. Circ. No. M73/2.

Bath, G. T.: 1972, Astrophys. J. 173, 121.

Bath, G. T.: 1973, Nature Phys. Sci. 246, 84.

Bath, G. T., Evans, W. D., and Pringle, J. E.: 1974a, Monthly Notices Roy. Astron. Soc. 166, 113.

Bath. G. T., Evans, W. D., and Papaloizou, J.: 1974b, Monthly Notices Roy. Astron. Soc. 167, 7P.

Beals, C. S. and Oke, J. B.: 1953, Monthly Notices Roy. Astron. Soc. 113, 530.

Blaauw, A: 1963, in Strand (ed.), Basic Astronomical Data, Univ. Chicago Press.

Biermann, P. and Hall, D. S.: 1973, Astron. Astrophys. 27, 249.

Blumenthal, G. R. and Tucker, W. H.: 1974, in Giacconi and Gursky (eds.), X-Ray Astronomy, Reidel, Dordrecht, Holland, p. 99.

Bond, H. E. and Landolt, A. U.: 1971, Publ. Astron. Soc. Pacific 83, 485.

Bond, H. E. and Tifft, W. G.: 1974, Publ. Astron. Soc. Pacific 86, 981.

Bopp, B. W., Gehrz, R. D., and Hackwell, J. A.: 1974, Publ. Astron. Soc. Pacific 86, 989.

Boyarchuk, A. A.: 1969, in L. Detre (ed.), Nonperiodic Phenomena in Variable Stars, Academic Press, Budapest, p. 395.

Bretz, M.: 1965, Colloq. IAU, Bamberg, p. 244.

Brickhill, A. J.: 1975, Monthly Notices Roy. Astron. Soc. 170, 405.

Burbidge, E. M. and Burbidge, G. R.: 1953, Astrophys. J. 118, 349.

Burrel, J. F. and Mould, J. R.: 1973, Publ. Astron. Soc. Pacific 85, 627.

Carlson, E. D. and Henize, K. G.: 1974, A strophys. J. Letters 188, L47.

Catchpole, R. M.: 1969, Monthly Notices Roy. Astron. Soc. 142, 119.

Chincarini, G: 1975 (private communication). 
Chodil, G., Mark, H., Rodrigues, R., and Swift, C. D.: 1968, Astrophys. J. Letters 152, L45.

Churms, J.: 1975 (private communication).

Code, A. D.: 1973, IAU Symp. 54, 131.

Conner, S. P., Evans, W. D., and Belian, R. D.: 1969, Astrophys. J. Letters 157, L157.

Copeland, H., Jensen, J. D., and Jorgensen, H. E.: 1970, Astron. Astrophys. 5, 12.

Cowley, A. P. and MacConnell, D. J.: 1972, Astrophys. J. Letters 176, L27.

Cowley, A. P., Crampton, D., Hutchings, J. B., and Marlborough, J. M.: 1975, Astrophys. J. 195, 413.

Cox, D. P. and Tucker, W. H.: 1969, Astrophys. J. 157, 1157.

Crawford, J. A. and Kraft, R. P.: 1956, Astrophys. J. 123, 44.

Eggen, O. J.: 1967, Mem. Roy. Astron. Soc. 70, 111.

Eggen, O. J.: 1968, Quart. J. Roy. Astron. Soc. 9, 332.

Eggen, O. J.: 1969, Astrophys. J. 157, 287.

Eggen, O. J. and Greenstein, J. L.: 1965, Astrophys. J. 141, 83.

Elliot, J. L. and Liller, W. M.: 1972, Astrophys. J. Letters 175, L69.

Elvey, C. T. and Babcock, H. W.: 1943, Astrophys. J. 97, 412.

Faulkner, J.: 1971, Astrophys. J. Letters 170, L99.

Faulkner, J.: 1974, in R. J. Tayler (ed.), Late Stages of Stellar Evolution, Reidel, Dordrecht, Holland, p. 155.

Faulkner, J., Flannery, B. P., and Warner, B.: 1972, Astrophys. J. Letters 175, L79.

Feast, M. W.: 1975 (private communication).

Feast, M. W. and Glass, I. S.: 1974, Monthly Notices Roy. Astron. Soc. 167, 81.

Flannery, B. P.: 1974, Ph.D. Thesis, Univ. of California.

Flannery, B. P.: 1975, Monthly Notices Roy. Astron. Soc. 170, 325.

Gallagher, J. S. and Code, A. D.: 1974, Astrophys. J. 189, 303.

Gallagher, J. S. and Holm, A. V.: 1974, Astrophys. J. Letters 189, L123.

Gallagher, J. S. and Oinas, A. V.: 1974, Publ. Astron. Soc. Pacific 86, 952.

Geisel, S. L., Kleinman, D. E., and Low, F. J.: 1970, Astrophys. J. Letters 161, L101.

Giacconi, R., Murray, S., Gursky, H., Kellogg, E., Schreier, E., Matilsky, T., Koch, D., and Tananbaum, H.: 1974, Astrophys. J. Suppl. 27, 37.

Graboske, H. C. and Grossman, A. S.: 1971, Astrophys. J. 170, 363.

Grant, G.: 1955, Astrophys. J. 122, 566.

Grant, G. and Abt, H. A.: 1959, Astrophys. J. 129, 323.

Greenstein, J. L.: 1954, Publ. Astron. Soc. Pacific 66, 79.

Greenstein, J. L.: 1960, in Greenstein (ed.), Stellar Atmospheres, Univ. Chicago Press, p. 676.

Greenstein, J. L.: 1966, Astrophys. J. 144, 496.

Greenstein, J. L. and Kraft, R. P.: 1959, Astrophys. J. 130, 99.

Greenstein, J. L. and Matthews, M. S.: 1957, Astrophys. J. 105, 85.

Greenstein, J. L., Sargent, A. I., and Huag, U.: 1970, Astron. Astrophys. 7, 1.

Greep, P.: 1942, Dissertation. Utrecht.

Grossman, A. S., Hays, D., and Graboske, H. C.: 1971, Astron. Astrophys. 30, 95.

Harries, J. R., Tuohy, I. T., Broderick, A. J., Fenton, K. B., and Luyendyk, A. P. J.: 1971, Nature 234, 149.

Harwood, J.: 1973, M.Sc. Thesis, University of Cape Town.

Heise, J., Brinkman, A. C., Schrijver, J., Mewe, R., Groneschild, E., and Den Boggende, A.: 1975 (preprint).

Henry, P., Cruddace, R., Lampton, M., Paresce, F., and Bowyer, S.: 1975, Astrophys. J. Letters 197, L117.

Herbig, G. H.: 1950, Publ. Astron. Soc. Pacific 62, 211.

Herbig, G. H.: 1960a, Astrophys. J. 131, 632.

Herbig, G. H.: 1960b, Astrophys. J. 132, 76.

Herbig, G. H.: 1964, in W. J. Luyten (ed.), First Conference on Faint Blue Stars, University of Minnesota, p. 100.

Herbig, G. H. and Rao, N. K.: 1972, Astrophys. J. 174, 401.

Herbig, G. H., Preston, G. W., Smak, J., and Paczynski, B.: 1965, Astrophys. J. 141, 617.

Herbst, W. and Ostriker, J. P.: 1971, Bull. Am. Astron. Soc. 3, 175.

Herbst, W., Hesser, J. E., and Ostriker, J. P.: 1974, Astrophys. J. 193, 679.

Hesser, J. E., Lasker, B. M., and Osmer, P. S.: 1974, Astrophys. J. 189, 315.

Hinderer, F.: 1949, Astron. Nachr. 277, 193.

Hjellming, R. M. and Wade, C. M.: 1970, Astrophys. J. Letters 162, L1.

Holm, A. V. and Gallagher, J. S.: 1974, A strophys. J. 192, 425. 
Humason, M. L.: 1938, Astrophys. J. 88, 237.

Humphries, C. M., Nandy, K., and Kontizas, E.: 1975, Astrophys. J. 195, 111.

Hutchings, J. B.: 1972, Monthly Notices Roy. Astron. Soc. 158, 177.

Ives, J. C., Sanford, P. W. and Bell-Burnell, J.: 1975, Nature (in press).

Johnson, H. L.: 1966, Ann. Rev. Astron. Astrophys. 4, 193.

Joy, A. H.: 1940, Publ. Astron. Soc. Pacific 52, 324.

Joy, A. H.: 1954, Astrophys. J. $120,377$.

Joy, A. H.: 1960, in J. L. Greenstein (ed.), Stellar Atmospheres, Univ. Chicago Press, p. 653.

Kemp, J. C., Swedlund, J. B., and Wolstencroft, R. D.: 1974, Astrophys. J. Letters 193, L15.

Kiplinger, A. L. and Nather, R. E.: 1975, Nature 225, 125.

Kopal, Z.: 1955, Ann. Astrophys. 18, 379.

Kraft, R. P.: 1975, private communication.

Kraft, R. P.: 1956, Carnegie Inst. Yearbook, Washington, p. 56.

Kraft, R. P.: 1958, Astrophys. J. 127, 625.

Kraft, R. P.: 1962, Astrophys. J. 135, 408.

Kraft, R. P.: 1963, in Kopal (ed.), Adv. Astron. Astrophys. 2, 43, Academic Press, New York.

Kraft, R. P.: 1964a, Astrophys. J. 139, 457.

Kraft, R. P.: 1964b, in W. J. Luyten (ed.), First Conference on Faint Blue Stars, University of Minnesota: The Observatory.

Kraft, R. P.: 1966, Trans. IAU XIIB, 519.

Kraft, R. P. and Demoulin, M.: 1967, Astrophys. J. Letters 150, L183.

Kraft, R. P. and Luyten, W. J.: 1965, Astrophys. J. 142, 1041.

Kraft, R. P., Krzeminski, W., and Mumford, G. S.: 1969, Astrophys. J. 158, 589.

Kruszewski, A.: 1967, Acta. Astron. 17, 297.

Krzeminski, W.: 1965, Astrophys. J. 142, 1051.

Krzeminski, W.: 1966, IAU Inf. Bull. Var. Stars No. 160.

Krzeminski, W.: 1972, Acta Astron. 22, 387.

Krzeminski, W. and Kraft, R. P.: 1964, Astrophys. J. 140, 921.

Krzeminski, W. and Smak, J.: 1971, Acta Astron. 21, 133.

Kuiper, G. P.: 1941, Astrophys. J. 93, 133.

Kukarkin, B. V. and Parenago, P. P.: 1934, Var. Star Bull. IV, No. 8, p. 44.

Kunkel, W. E.: 1970, Astrophys. J. 161, 503.

Landau, C. D. and Lifshitz, E. M.: The Classical Theory of Fields, Reading, Mass.

Landstreet, J. D. and Angel, J. R. P.: 1971, Astrophys. J. Letters 165, L67.

Lasker, B. and Hesser, J.: 1969, Astrophys. J. Letters 158, L171.

Lasker, B. and Hesser, J.: 1971, Astrophys. J. Letters 163, L89.

Lawrence, G. M., Ostriker, J. P., and Hesser, J. E.: 1967, Astrophys. J. Letters 148, 161.

Lewin, W. H. G., Clark, G. W., and Smith, W. B.: 1968, Astrophys. J. Letters 152, L49.

Liller, M. H.: 1962, Harv. Coll. Obs. Announcement Card 1576.

Lin, D. N. C.: 1975, Monthly Notices Roy. Astron. Soc. 170, 379.

Linnell, A. P.: 1949, Sky Telesc. 8, 166.

Lortet-Zuckermann, M.-C.: 1967, Compt. Rend. Acad. Sci. Paris 265, 826.

Lortet, M.-C.: 1968, IAU Colloq., Budapest, p. 381.

Luyten, W. J. and Hughes, H. S.: 1965, Publ. Univ. Minnesota, No. 36.

Lynds, C. R.: 1967, Astrophys. J. Letters 149, L41.

Mandel, O.: 1965, Per. Zuezdy 15, 474.

Marino, B. F. and Walker, W. S. G.: 1973, Southern Stars 24, 126.

Marino, B. F. and Walker, W. S. G.: 1974, Inf. Bull. Var. Stars No. 864.

Markaryan, B. E.: 1967, Astrofisik 3, 511.

Matilsky, T. A., Giacconi, R., Gursky, H., Kellogg, E., and Tananbaum, H. D.: 1972, Astrophys. J. 174, L53.

Matthews, W. G. and Sandage, A. R.: 1963, Astrophys. J. 138, 30.

McLaughlin, D. B.: 1953, Astrophys. J. 117, 279.

McLaughlin, D. B.: 1960, in J. L. Greenstein (ed.), Stellar Atmospheres, Univ. Chicago Press, p. 585.

Mestel, L.: 1952, Monthly Notices Roy. Astron. Soc. 112, 598.

Mirzoyan, L. V. and Kalloglyan, N. L.: 1965, Astrophys. 1, 203.

Moffat, A. F. J. and Vogt, N.: 1975, Astron. Astrophys. Suppl. Ser. 20, 85.

Moffett, T. J. and Barnes, T. G.: 1974, Astrophys. J. 194, 141.

Morton, D. C. and Adams, T. F.: 1968, Astrophys. J. 151, 611.

Mumford, G. S.: 1964, Astrophys. J. 139, 476.

Mumford, G. S.: 1967a, Publ. Astron. Soc Pacific 79, 283. 
Mumford, G. S.: 1967b, IAU Inf. Bull. Var. Stars No. 197.

Mumford, G. S.: 1967c, Astrophys. J. Suppl. 15, 1.

Mumford, G. S.: 1971, Astrophys. J. 165, 369.

Mumford, G. S. and Krzeminski, W.: 1969, Astrophys. J. Suppl. 18, 429.

Münch, G.: 1968, in Middlehurst and Aller (eds.), Nebulae and Interstellar Matter, Univ. Chicago Press, p. 365.

Mustel, E. R. and Boyarchuk, A. A.: 1970, Astrophys. Space Sci. 6, 183.

Nather, R. E. and Robinson, E. L.: 1974, Astrophys. J. 190, 637.

Nather, R. E. and Warner, B.: 1969, Monthly Notices Roy. Astron. Soc. 143, 145.

Nather, R. E. and Warner, B.: 1971, Monthly Notices Roy. Astron. Soc. 152, 209.

Nelson, M. R.: 1975, Astrophys. J. Letters 196, L113.

Novick, R. and Woltjer, L.: 1975, Astrophys. Letters 16, 67.

Osaki, Y.: 1974 (preprint).

Paczynski, B.: 1963, Publ. Astron. Soc. Pacific 75, 278.

Paczynski, B.: 1965a, Acta Astron. 15, 197.

Paczynski, B.: 1965b, Acta Astron. 15, 305.

Paczynski, B.: 1967, Acta Astron. 17, 287.

Paczynski, B.: 1971, Ann. Rev. Astron. Astrophys. 9, 183.

Paczynski, B. and Sienkiewicz, R.: 1972, Acta Astron. 22, 73.

Payne-Gaposchkin, C.: 1975 (private communication).

Payne-Gaposchkin, C.: 1946, Astrophys. J. 104, 362.

Payne-Gaposchkin, C.: 1957, in The Galactic Novae, North-Holland Publ. Co., Amsterdam.

Payne-Gaposchkin, C. and Boyd, C.: 1946, Astrophys. J. 104, 357.

Piotrowski, S. L.: 1975, Acta Astron. 25, 21.

Piotrowski, S. L. and Ziołkowski, K.: 1970, Astrophys. Space Sci. 8, 66.

Plavec, M. and Kratochvil, P.: 1964, Bull. Astron. Inst. Czech. 15, 165.

Pottasch, S. R.: 1959, Ann. Astrophys. 22, 394.

Pottasch, S. R.: 1967, Bull. Astron. Soc. Neth. 19, 227.

Prendergast, K. H.: 1960, Astrophys. J. 132, 162.

Prendergast, K. H. and Taam, R. E.: 1974, Astrophys. J. 189, 125.

Pringle, J. E.: 1975, Monthly Notices Roy. Astron. Soc. 170, 633.

Pringle, J. E. and Rees, M. J.: 1972, Astron. Astrophys. $21,1$.

Pringle, J. E. and Webbink, R. F.: 1965 (preprint).

Rao, U. R., Chitnis, E. V., Prakasarao, A. S., and Jayanthi, U. B.: 1969, Astrophys. J. Letters 157 , L127.

Rappaport, S., Cash, W., Doxsey, R., McClintock, J., and Moore, G.: 1974, Astrophys. J. Letters 187, L5.

Robinson, E. L.: 1973a, Astrophys. J. 180, 121.

Robinson, E. L.: 1973b, Astrophys. J. 181, 531.

Robinson, E. L.: 1973c, Astrophys. J. 183, 193.

Robinson, E. L.: 1973d, Astrophys. J. 186, 347.

Robinson, E. L.: 1974, Astrophys. J. 193, 191.

Robinson, E. L., Nather, R. E., and Kiplinger, A.: (preprint).

Rose, W. K. and Smith, R. L.: 1972, Astrophys. J. 172, 699.

Sandage, A.: 1972, Astrophys. J. 178, 1.

Saslaw, W. C.: 1968, Monthly Notices Roy. Astron. Soc. 138, 337.

Schild, R. E.: 1969, Astrophys. J. 157, 709.

Schmidt-Kaler, Th.: 1962, Verolffentl. der Remeis-Sternwarte, Bamburg No. 34.

Silk, J.: 1973, Astrophys. J. 181, 747.

Smak, J.: 1967, Acta Astron. 17, 255.

Smak, J.: 1969, Acta Astron. 19, 155.

Smak, J.: 1970, Acta Astron. 20, 311

Smak, J.: 1971 a, Acta Astron. 21, 15.

Smak, J.: 1971b, Acta Astron. 21, 467.

Smak, J.: 1971 c, IAU Colloq. 15, Bamberg, p. 248.

Smak, J.: 1972, Acta Astron. 22, 1.

Smak, J. and Stepien, K.: 1969, in L. Detre (ed.), Nonperiodic Phenomena in Variable Stars, Budapest, Academic Press, p. 335.

Sofia, S.: 1972, Astrophys. J. Letters, 175, L113.

Sorenson, S.-A., Matsuda, T. and Sakurai, T.: 1975, Astrophys. Space Sci. 33, 465.

Sparks, W. M. and Starrfield, S.: 1973, Monthly Notices Rov. Astron. Soc. 164, 1 P. 
Stalio, R.: 1971, in M. Hack (ed.), Proc. Third Colloq. on Astrophysics, Trieste, p. 28.

Starrfield, S., Truran, J. W., Sparks, W. M., and Kutter, G. S.: 1972, Astrophys. J. 176, 169.

Starrfield, S., Sparks, W. M., and Truran, J. W.: 1974a, Astrophys. J. Suppl. 28, 247.

Starrfield, S., Sparks, W. M., and Truran, J. W.: 1974b, Astrophys. J. 192, 647.

Stephenson, C. B.: 1967, Publ. Astron. Soc. Pacific 79, 584.

Stephenson, C. B., Sanduleak, N., and Schild, R. E.: 1968, Astrophys. Letters 1, 247.

Sterne, T. E. and Cambell, L.: 1934, Harvand Ann. 90, 189.

Strand, K. Aa.: 1948, Astrophys. J. 107, 106.

Strohmeier, W.: 1962, Inf. Bull. Var. Stars No. 15.

Struve, O.: 1948, Astrophys. J. 108, 153.

Swedlund, J. B., Kemp, J. C., and Wolstencroft, R. D.: 1974, Astrophys. J. 193, L11.

Szkody, P.: 1974, Astrophys. J. Letters 192, L75.

Ulmer, M. P., Baity, W. A., Wheaton, W. A., and Peterson, L. E.: 1973, Astrophys. J. Letters 184, L117.

van Houten, C. J.: 1966, Bull. Astron. Inst. Neth. 18, 439.

van Maanen, A.: 1926, Publ. Astron. Soc. Pacific 38, 325.

van Maanen, A.: 1938, Astrophys. J. 87, 424.

Vogt, N.: 1974, Astron. Astrophys. 36, 369.

Vogt, N.: 1975 (private communication).

Vojkhanskaya, N. F.: 1973a, Astron. Tsirk. No. 801, p. 5.

Vojkhanskaya, N. F.: 1973b, Astrofiz. Issled., Izv. Spets. Astrofiz. Obs. 5, 89.

Walker, A. R.: 1975 (private communication).

Walker, M. F.: 1954, Publ. Astron. Soc. Pacific 66, 230.

Walker, M. F.: 1956, Astrophys. J. 123, 68.

Walker, M. F.: 1957, IAU Symp. 3, 46.

Walker, M. F.: 1958, Astrophys. J. 127, 319.

Walker, M. F.: 1961, Astrophys. J. 134, 171.

Walker, M. F.: 1963a, Astrophys. J. 137, 485.

Walker, M. F.: 1963b, Astrophys. J. 138, 313.

Walker, M. F.: 1966, Adv. Electr. 22B, 761.

Walker, M. F.: 1971, IAU Colloq. 15, Bamberg, p. 243.

Walker, M. F. and Chincarini, G.: 1968, Astrophys. J. 154, 157.

Walker, M. F. and Herbig, G. H.: 1954, Astrophys. J. 120, 278.

Walker, M. F. and Reagan, G. H.: 1971, Inf. Bull. Var. Stars No. 544.

Wallerstein, G.: 1962, Astrophys. J. 137, 991.

Wallerstein, G.: 1963, Publ. Astron. Soc. Pacific 75, 26.

Warner, B.: 1971, in L. Detre (ed.), New Directions and New Frontiers in Variable Star Research, Bamberg, p. 144.

Warner, B.: 1972a, IAU Colloq. 15, Bamberg, p. 144.

Warner, B.: 1972b, Monthly Notices Roy. Astron. Soc. 160, 35P.

Warner, B.: 1972c, Monthly Notices Roy. Astron. Soc. 158, 425.

Warner, B.: 1973a, Monthly Notices Roy. Astron. Soc. 163, 25P.

Warner, B.: 1973b, Monthly Notices Roy. Astron. Soc. 162, 189.

Warner, B.: 1973c, Monthly Notices Astron. Soc. South Africa 32, 120.

Warner, B.: 1974a, Monthly Notices Roy. Astron. Soc. 168, 235.

Warner, B.: 1974b, Monthly Notices Roy. Astron. Soc. 167, 47P.

Warner, B.: 1974c, Monthly Notices Astron. Soc. South Africa 33, 133.

Warner, B.: 1975a, Montlhly Notices Roy. Astron. Soc. 170, 219.

Warner, B.: 1975b, Monthly Notices Roy. Astron. Soc. (in press).

Warner, B.: 1975c (in preparation).

Warner, B. and Brickhill, A. J.: 1974, Monthly Notices Roy. Astron. Soc. 166, 673.

Warner, B. and Brickhill, A. J.: 1975, Monthly Notices Roy. Astron. Soc. (in press).

Warner, B. and Nather, R. E.: 1971, Monthly Notices Roy. Astron. Soc. 152, 219.

Warner, B. and Nather, R. E.: 1972a, Monthly Notices Roy. Astron. Soc. 156, 305.

Warner, B. and Nather, R. E.: 1972b, Sky Telesc. 43, 82.

Warner, B. and Nather, R. E.: 1972c, Monthly Notices Roy. Astron. Soc. 159, 429.

Warner, B. and Peters, W. L.: 1972, Monthly Notices Roy. Astron. Soc. 160, 15.

Warner, B. and Robinson, E. L.: 1972a, Monthly Notices Roy. Astron. Soc. 159, 101.

Warner, B. and Robinson, E. L.: 1972b, Nature Phys. Sci. 239, 2.

Warner, B. and Thackeray, A. D.: 1975, Monthly Notices Roy. Astron. Soc. 172, 433.

Warner, B. and van Citters, G. W.: 1974, Observatory 94, 116. 
Warner, B., Peters, W. L., Hubbard, W. B., and Nather R. E.: 1972, Monthly Notices Royal Astron. Soc. $159,321$.

Weaver, H.: 1974, in Highlights of Astronomy 3, 509.

Wegner, G.: 1972, Proc. Astron. Soc. Australia 2, 107.

Wellman, P.: 1952, Z. Astrophys. 31, 123.

Westphal, J. A., Sandage, A., and Kristian, J.: 1968, Astrophys. J. 154, 139.

Young, A. and Nelson, B.: 1972, Astrophys. J. 173, 653.

Zinner, E.: 1938, Astron. Nachr. 265, 345.

Zuckermann, M.-C.: 1954, Ann. Astrophys. 17, 243.

Zuckermann, M.-C.: 1961, Ann. Astrophys. 24, 431.

Zwicky, F.: 1965, in 3rd Colloq. on Variable Stars, Bamberg, Astr. Inst. Univ. Erlangen-Nurnberg IV, No. 40, p. 169. 